\title{
Distribution of deep-sea benthic foraminifera in the Neogene of Blake Ridge, NW Atlantic Ocean
}

\author{
KUPPUSAMY MOHAN ${ }^{1}$, ANIL K. GUPTA ${ }^{1, *}$ \& AJOY K. BHAUMIK ${ }^{2}$ \\ ${ }^{1}$ Department of Geology \& Geophysics, Indian Institute of Technology Kharagpur - 721 302, India \\ ${ }^{2}$ Department of Applied Geology, Indian School of Mines Dhanbad, Jharkhand - 826 004, India \\ ${ }^{*}$ Corresponding author (e-mail: anilg@gg.iitkgp.ernet.in)
}

\begin{abstract}
This study describes and illustrates the evolution of deep-sea benthic foraminifera from the Blake Ridge during the late Neogene. In total, 305 species of benthic foraminifera belonging to 107 genera were identified. The Blake Ridge receives fine-grained nannofossil-bearing hemipelagic sediments, transported from the Canadian continental margin by the Deep Western Boundary Undercurrent (DWBUC). We thus presume that changes in benthic foraminifera at Ocean Drilling Program (ODP) sites 991A, 994C, 995A and B and 997A reflect mainly changes in the intensity of the DWBUC, which is closely related to North Atlantic Deep Water (NADW) production. However, the dominance of Uvigerina peregrina, U. proboscidea and Cassidulina carinata during the late Miocene in all the holes suggests an increased influence of Southern Component Waters in the Blake Ridge region. During the early Pliocene (4.8-2.8 Ma) in all the sites benthic faunal assemblages suggest that there was an increased transport of organic-rich sediments by the DWBUC from the Canadian margin to the Blake Ridge, driven by increased production of NADW. During this time the species diversity (Sanders' rarefied values) was low. In the younger interval (since $2.8 \mathrm{Ma}$ ), the faunal data suggest less transport of organic-rich sediments to the Blake Ridge, which appears to be related to weakening of the DWBUC during cold intervals. An increase in species diversity at $3 \mathrm{Ma}$ probably resulted from decreased population of bacteria due to low organic matter and/or less competition. In the late Pleistocene (c. $0.6 \mathrm{Ma})$, Stilostomella lepidula became extinct in all the studied holes, suggesting that this species may have possessed a mode of feeding which no longer existed in the cold, well-oxygenated oceans of the present. J. Micropalaeontol. 30(1): 33-74, May 2011.
\end{abstract}

\begin{abstract}
Supplementary material: Relative abundances of important benthic foraminifera and Sanders' rarefied values used in this study from ODP Holes 991A, 994C, 995A, 995B and 997A, Blake Ridge, NW Atlantic Ocean are available at: http://www.geolsoc.org.uk/SUP18457
\end{abstract}

KEYWORDS: Benthic foraminifera, late Neogene, Blake Ridge, North Atlantic Deep Water

\section{INTRODUCTION}

Benthic foraminifera are an important source of information on past environmental variability. This group occupies perched epibenthic to deep infaunal microhabitats, utilizing a variety of trophic mechanisms. Benthic foraminifera are able to survive/ proliferate in a wide range of marine environments, including extreme ecosystems, such as oligotrophic abyssal plains (Coull et al., 1977) or hydrothermal vents (Sen Gupta \& Aharon, 1994), cold seeps (Rathburn et al., 2000; Bernhard et al., 2001; Robinson et al., 2004) and deep sea trenches (Akimoto et al., 2001).

Benthic foraminiferal distributions are closely tied to the organic carbon flux of the sea floor and organic carbon content of the sediment (Caralp, 1984, 1989a, b; Van der Zwaan et al., 1990; Jorissen et al., 1992; Gooday, 1993; Schmiedl et al., 1997; De Stigter et al., 1998; Gupta \& Thomas, 1999, 2003; Singh \& Gupta, 2004), and the dissolved oxygen content of bottom water and pore water oxygenation (Corliss \& Emerson, 1990; Hermelin \& Shimmield, 1990; Gooday, 1994; Jannink et al., 1998). In addition, there are other factors that significantly influence benthic foraminiferal distribution, such as the nature of organic matter (degraded, labile, refractory, etc.), trace elements in the sediments and presence of methane (Wefer et al., 1994; Rathburn et al., 2000; Fontanier et al., 2005; Bhaumik \& Gupta, 2007).

The Blake Ridge in the westernmost part of the North Atlantic Ocean (Fig. 1) is a sediment drift, adjacent to two important components of the Atlantic Meridional overturning circulation: the warm, saline Gulf Stream and the Deep Western Boundary Undercurrent (DWBUC). The ridge sediments largely consist of fine-grained nannofossil-bearing hemipelagic sediments, transported from the Canadian continental margin by

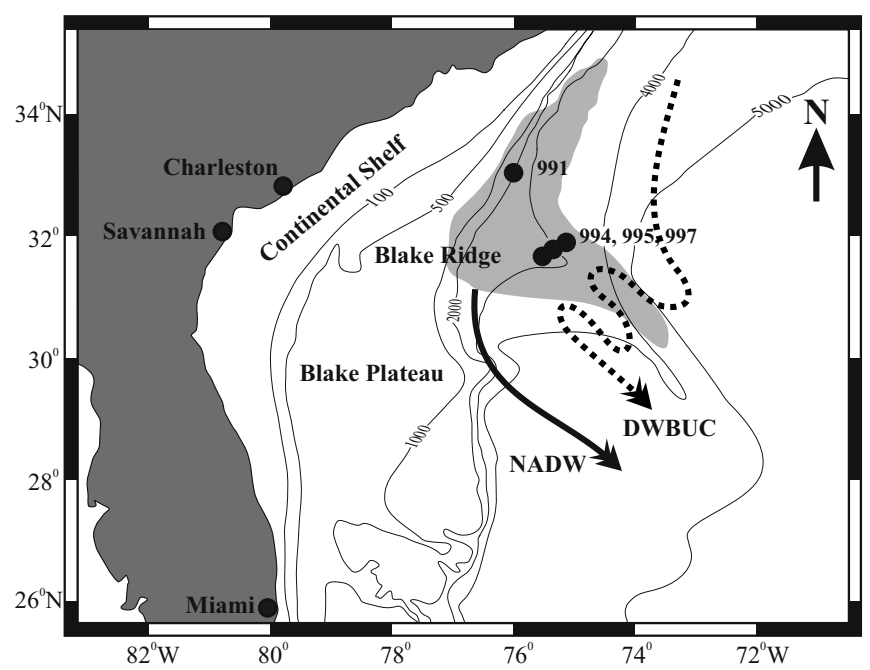

Fig. 1. Map showing locations of ODP holes from Blake Ridge, NW Atlantic Ocean, Leg 164. Shaded area represents the inferred distribution of bottom simulating reflector (BSR). Contours are in metres. NADW, North Atlantic Deep Water (continuous arrow); DWBUC, Deep Western Boundary Undercurrent (broken arrow). 


\begin{tabular}{|c|c|c|c|c|c|}
\hline Hole & $991 \mathrm{~A}$ & $994 \mathrm{C}$ & $995 \mathrm{~A}$ & $995 B$ & 997A \\
\hline Latitude & $32^{\circ} 59.018^{\prime} \mathrm{N}$ & $31^{\prime} 47^{\prime} 139 \mathrm{~N}$ & $31^{\circ} 48.210^{\prime} \mathrm{N}$ & $31^{\circ} 48.217^{\prime} \mathrm{N}$ & $31^{\prime} 50^{\prime} 588 \mathrm{~N}$ \\
\hline Longitude & $75^{\circ} 55.801^{\prime} \mathrm{W}$ & $75^{\prime} 32^{\prime} 753 \mathrm{~W}$ & $75^{\circ} 31.343^{\prime} \mathrm{W}$ & $75^{\circ} 31.336^{\prime} \mathrm{W}$ & $75^{\prime} 28^{\prime} 118 \mathrm{~W}$ \\
\hline Penetration (mbsf) & 56.6 & 703.5 & 704.6 & 700 & 434.3 \\
\hline & 5.9 Ma to Recent & 6.8 Ma to Recent & 6.6 Ma to Recent & 4.9 Ma to Recent & 5.4 Ma to Recent \\
\hline Age (Ma) & $1-\ldots$ & - & ate Miocene to Rece & - & 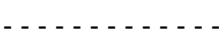 \\
\hline
\end{tabular}

Table 1. Summary of ODP holes 991A, 994C, 995A, 995B and 997A, including water depth, location, penetration and stratigraphic range of the studied sequence.

the DWBUC (Markl et al., 1970; Reynolds et al., 1999; Balsam \& Damuth, 2000).

At present, the flanks of the Blake Ridge above c. $3500 \mathrm{~m}$ are covered by the Northern Component Waters (NCW), carried by the DWBUC to the south, with a density of $c .27 .88 \mathrm{~kg} \mathrm{~m}^{-3}$ and a dissolved oxygen concentration of $c .6 .3 \mathrm{ml} \mathrm{l}^{-1}$ (Bower \& Hunt, 2000). At depths greater than $c .4000 \mathrm{~m}$, the ridge is covered by Southern Component Waters (SCW), mainly fed by the Antarctic Bottom Water (AABW). This deep bottom water, however, consists of a varying mixture of NCW (up to 90\%) and SCW (Stahr \& Sanford, 1999). There have been few studies to reconstruct the relative volume of $\mathrm{NCW}$ and SCW during earlier time periods. Frank et al. (2002) and Reynolds et al. (1999) used $\mathrm{Nd}$ and $\mathrm{Pb}$ isotopes to argue that the export of the SCW was strong prior to $3 \mathrm{Ma}$, and linked changes in $\mathrm{Pb}$ isotope values after $3 \mathrm{Ma}$ and more dramatic changes since $1.8 \mathrm{Ma}$ to the North Atlantic circulation as related to the Northern Hemisphere glaciation (NHG).

The Blake Ridge archives a continuous and thick sedimentary record, providing ample opportunities to examine a link between benthic foraminiferal populations, DWBUC and deep water masses influenced by NCW and SCW. To understand this relationship, we analysed population trends in dominant benthic foraminifera and Sanders' rarefied values from five Ocean Drilling Program (ODP) holes, 991A, 994C, 995A and B and 997A, drilled during Leg 164 on the Blake Ridge, NW Atlantic Ocean. We also document and illustrate important benthic foraminiferal species with scanning electron micrographs that give an idea about the state of preservation of benthic fauna in the Blake Ridge.

\section{LOCATION AND OCEANOGRAPHIC SETTING}

Ocean Drilling Program (ODP) holes 991A, 994C, 995A, 995B and 997A (Leg 164) are located on the Blake Ridge, c. $200 \mathrm{~km}$ off the east coast of the USA in the NW Atlantic Ocean (Fig. 1, Table 1). These holes lie in a tectonically inactive setting close to the passive margin and were not affected by late Cenozoic tectonic activity or by fluid flow along major faults in the sediments (Wood \& Ruppel, 2000). Site 991, hole A lies near the upslope flank of the Cape Fear Diapir, where sediments that may formerly have contained gas hydrate have been disturbed (Paull et al., 1996). This hole has three or more discrete mass-transport zones of different ages ranging from the Miocene to the Pleistocene, punctuated by undisturbed sediment intervals. The geological setting of Site 995 is similar to that of Sites 994 and 997. These sites are located on the southern flank of the Blake Ridge - a Neogene sediment drift (Tucholke \& Mountain,
1979). The depth and speed of the DWBUC have varied on glacial-interglacial time-scales, with shallower depth and slow speed related to the increased influence of SCW (AABW) during the late Miocene to the Pleistocene (Ledbetter \& Balsam, 1985). The depth of the contact between NCW (above) and SCW (below) has changed significantly over time, generally shallowing by more than $2000 \mathrm{~m}$ during glacial intervals, so that the DWBUC's zone of maximum flow speed shifted to a depth of less than $2500 \mathrm{~m}$ (Evans \& Hall, 2008). The major increase in DWBUC depth during interglacials and speed correlates with the resumption of North Atlantic Deep Water (NADW) production (Shackleton et al., 1983).

The depositional environment at Blake Ridge is strongly influenced by glacial-interglacial switches in the strength and position of the DWBUC that preferentially eroded sediments from the Canadian continental margin (Laine et al., 1994) and led to the sediment drifts in the Blake-Bahama Outer Ridge (BBOR) region (Franz \& Tiedemann, 2002). These drift deposits consist of cyclic alternations between carbonate-rich and clayrich sediments with higher carbonate concentrations during interglacial stages. Because NADW circulation enhances speed of DWBUC, and NADW production is higher during interglacials (Franz \& Tiedemann, 2002), it is expected that the DWBUC will intensify sediment transport from the Canadian continental margin during interglacial periods.

The modern lysocline in the Blake Ridge area lies between $4000 \mathrm{~m}$ and $4350 \mathrm{~m}$ water depth (Balsam, 1983). The only tectonic event that occurred adjacent to the study area is the closing of the Panama Isthmus around $4 \mathrm{Ma}$, which drove significant changes in the thermohaline circulation of the North Atlantic (Haug \& Tiedemann, 1998).

\section{MATERIALS AND METHODS}

One thousand five hundred and sixteen (1516) core samples were analysed, which were provided under request numbers 16030A, $16030 \mathrm{~B}$ and $16030 \mathrm{C}$ to AKG. From hole $991 \mathrm{~A}$ we analysed 179 samples, with a total length of $56.6 \mathrm{~m}$ below seafloor (mbsf), ranging from 5.9 Ma to the Recent; from hole 994C, 441 samples (up to $703.5 \mathrm{mbsf}$ ), covering the last $6.8 \mathrm{Ma}$; from hole 995A, 599 samples (up to $704.10 \mathrm{mbsf}$ ), ranging from 6.6 Ma to the Recent; from hole 995B, 59 samples (up to $453.06 \mathrm{mbsf}$ ), with a total duration of $4.9 \mathrm{Ma}$; and from hole 997A, 238 samples (up to $434.3 \mathrm{mbsf}$ ), spanning the last 5.4 Ma (Table 1).

Samples were processed with the necessary precautions to avoid contamination using the standard procedures (Gupta \& Thomas, 1999). Samples were soaked in clean tap water with few drops of hydrogen peroxide $\left(\mathrm{H}_{2} \mathrm{O}_{2}\right)$ of 3-5\% concentration for 
8-12 hours and were washed over a $63 \mu \mathrm{m}$ size sieve. The washed samples were dried in an electric oven at $c .50^{\circ} \mathrm{C}$ and transferred into labelled glass vials. We generated benthic foraminiferal census data from an aliquot of c. 300 specimens from the $>125 \mu \mathrm{m}$ size fraction. The specimens were counted, identified, their stratigraphic ranges plotted and percentages calculated (data available at www.pangaea.de). Sanders' rarefied values were calculated following the procedure described in Singh \& Gupta (2004). All samples are catalogued in the Paleoceanography and Paleoclimatology Laboratory of the Department of Geology \& Geophysics, Indian Institute of Technology, Kharagpur. Scanning electron micrographs were taken at the SEM laboratory of the Department of Geology \& Geophysics and Central Research Facility (CRF), Indian Institute of Technology, Kharagpur.

Ages for every sample were calculated based on interpolation between nannofossil datums (Okada, 2000) using the time-scale of Berggren et al. (1995a, b). For taxonomic identification, we largely followed Barker (1960), Boltovskoy (1978), Srinivasan \& Sharma (1980), Boersman (1990), Gupta (1994), Jones (1994) and numerous other relevant publications from the Atlantic Ocean. The generic identification is based on Loeblich \& Tappan $(1964,1988)$.

\section{RESULTS}

We identified 305 species of benthic foraminifera belonging to 107 genera. Out of these, 158 species belonging to 83 genera are shown in Plates 1-13. These species are listed in Appendix A. Some 262 species were identified at hole 991A, 220 species at hole $994 \mathrm{C}, 245$ species were recorded at hole 995A, 148 species at hole $995 \mathrm{~B}$ and 160 species at hole 997A. Table 2 shows the dominant species and their highest abundances in the studied ODP holes. Most of the species are long-ranging and common in all holes. A few, such as Bulimina aculeata, Cassidulina laevigata, Fursenkoina rotundata, Melonis barleeanum, M. pompilioides and Nonionella auris, show continuous occurrences coinciding with the beginning of NHG at c. 3 Ma (Fig. 2). The studied sequences do not appear to have been under the influence of the lysocline or Carbonate Compensation Depth (CCD) during the late Neogene, as there are no dissolution marks on the benthic foraminifera.

The most dominant benthic foraminiferal species at the studied ODP holes include Astrononion stelligerum, A. umbilicatulum, Cassidulina carinata, Globocassidulina obtusa, G. subglobosa, Epistominella exigua, Epistominella sp., Uvigerina peregrina, U. proboscidea, Melonis barleeanum, Pyrgo murrhina, Quadrimorphina laevigata and Stilostomella lepidula (Figs 3-6). Their percentages show important switches in their populations which could be helpful in understanding changes in the intensity of the DWBUC during the late Miocene to the Pleistocene (Figs 3-6). Figures 3 and 5 show percentages of individual species from site 991, while Figures 4 and 6 show combined census data of these species at sites 994, 995A and B and 997, since these sites are closely located. The species Cassidulina carinata, Uvigerina peregrina, U. proboscidea and Quadrimorphina laevigata show episodic occurrences at sites 994, 995 and 997 (Figs 4, 6), whereas at site 991 these species show secular trends, with less significant populations. Globocassidulina obtusa and Epistominella sp. show higher abundances in the Pliocene (5-3 Ma) and during 1.8-1.0 Ma at site 991, whereas at sites 994, 995 and 997 these species are abundant in the younger interval with more dominance during 1.8-1.0 Ma. At sites 994, 995 and 997, Uvigerina proboscidea and Globocassidulina subglobosa show higher abundances during 6.8 to $3.0 \mathrm{Ma}$, Astrononion stelligerum appears at c. 3.2 Ma, A. umbilicatulum shows higher abundances during 2.6-0.6 Ma, Epistominella exigua and Pyrgo murrhina show low abundances during 5-3 Ma and Melonis barleeanum remains abundant throughout the studied section, with a higher population during 5-3.5 Ma (Figs 4, 6). At site 991, Epistominella exigua and Pyrgo murrhina are also rare in the Pliocene, showing an inverse relationship with Globocassidulina obtusa.

Sanders' rarefied values show a major increase at c. 5.3 Ma and a second increase at c. $3 \mathrm{Ma}$ at hole 991A, coinciding with the onset of NHG (Zachos et al., 2001; Fig. 7). At holes 994C, 995A and 997A, these values show a major increase at c. $3 \mathrm{Ma}$ with abrupt changes in the younger interval (Fig. 7).

\section{ECOLOGICAL PREFERENCES OF BENTHIC FORAMINIFERA}

Benthic foraminifera live in variable ecological settings depending on their physiological and food requirements. Some species prefer to live in eutrophic, low-oxygen conditions, whereas others prefer to live in oligotrophic, better oxygenated conditions. Some are opportunists, whereas many species of benthic foraminifera are specialists. The following text details modern ecological preferences of the different benthic foraminiferal species used in the present study for palaeoceanographic reconstructions.

Astrononion umbilicatulum in the Red Sea shows a preference for high salinity waters (Gupta, 1994). This species is found during low productivity intervals on the Ontong-Java Plateau (Burke et al., 1993; Gupta, 1997). Astrononion umbilicatulum (synonymous with $A$. echolsi) has been found to be associated with lowest primary productivity and a well-ventilated water column in the Gulf of Aden (Almogi-Labin et al., 2000). This species prefers a well-ventilated deep-sea environment with low organic carbon flux in the southeastern Indian Ocean (Singh \& Gupta, 2004). Little or nothing is known about the ecological preference of Astrononion stelligerum. Its rare occurrence and similar population trend with $A$. umbilicatulum in this study suggests that this species prefers conditions identical to those of A. umbilicatulum.

Cassidulina carinata is a cosmopolitan, epifaunal to shallow infaunal, detrivorus, opportunistic taxon (Nees \& Struck, 1999; Hayward, 2002) and has been reported from diverse environments (Qvale \& Van Weering, 1985). This species responds positively to increasing input of fresh phytodetritus or labile organic matter to the ocean floor (Fontanier et al., 2003). In the Mediterranean Sea, C. carinata requires relatively eutrophic conditions of $>3 \mathrm{~g}$ of labile carbon $\mathrm{m}^{-2}$ year $^{-1}$ to flourish (De Rijk et al., 2000). In the Indian Ocean, an assemblage dominated by $C$. carinata and Gyroidinoides nitidula reflects an environment with intermediate organic flux and intermediate to high seasonality (Gupta \& Thomas, 2003). In addition, association of this species with Uvigerina proboscidea in the Indian Ocean indicates low oxygen conditions with continuous high food supply (Gupta, 1997; Gupta \& Thomas, 1999). Cassidulina carinata is common in the lower part of the oxygen minimum zone (OMZ) 


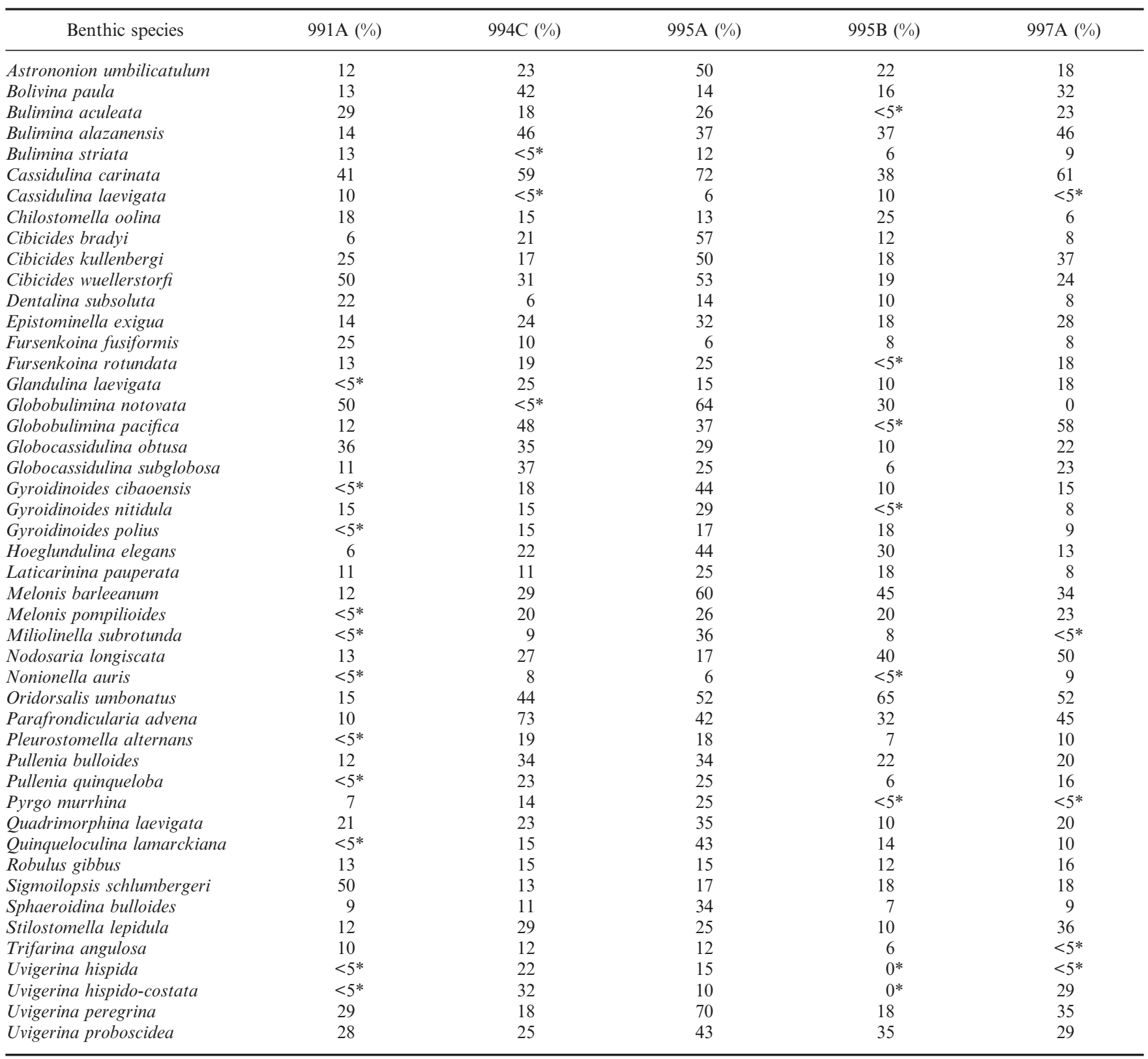

* Species with negligible percentages

Table 2. List of 47 dominant species of benthic foraminifera observed in the studied ODP holes, NW Atlantic Ocean, with their maximum percentages in that hole.

off the Pakistan continental and Oman margins (Hermelin \& Shimmield, 1990; Jannink et al., 1998). In the South Atlantic Ocean, it has been found correlated with the highest productivity and high organic carbon flux (Mackensen et al., 1995) and in the southeastern Arabian Sea this species is associated with high food supply (Gupta \& Thomas, 1999). Dominance of C. carinata in bathyal environments testifies to the broad habitat preference of this species and also its association with cold temperatures which prevail in bathyal settings below the thermocline (Hayward, 2002).

Epistominella exigua is a cosmopolitan species which feeds opportunistically on phytodetritus, deposited seasonally on the sea floor and usually associated with elevated oxygen concentrations (Gooday, 1988, 1993; Kurbjeweit et al., 2000; Gupta \& Thomas, 2003). Earlier studies observed dominance of E. exigua in the eastern and southern Indian Ocean at abyssal depths (Corliss, 1983; Peterson, 1984). Gooday (1993) correlated increased abundance of E. exigua with seasonal pulses of food supply. Also, in the eastern Indian Ocean, these food fluxes were related to the monsoonal climate where E. exigua thrives in deep environments with a temperature of $c .2 .5^{\circ} \mathrm{C}$ and oxygen of $>3.5 \mathrm{ml} \mathrm{l}^{-1}$ (Murgese \& De Deckker, 2005). Epistominella exigua has an advantage over other species when there is an input of fresh, labile organic matter (Caralp, 1989b; Gooday, 1994). The microhabitat preference 


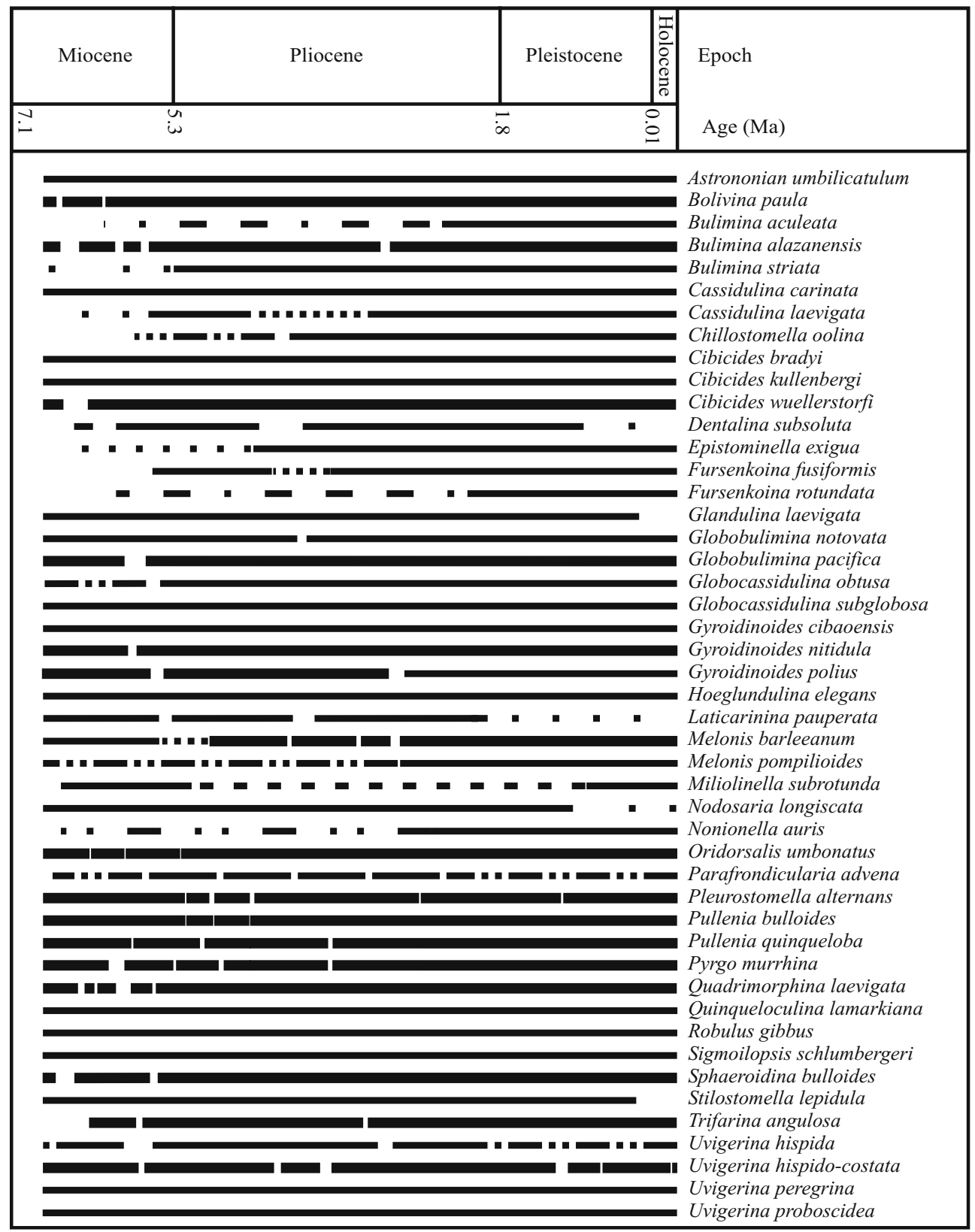

Fig. 2. Stratigraphic ranges of 47 dominant species of benthic foraminifera in the study area.

of Epistominella sp. is not well constrained, though it is quite abundant in Blake Ridge sediments following NHG.

Globocassidulina obtusa has been found associated with an assemblage linked to high productivity and low oxygen environments (Gupta \& Thomas, 1999). Singh \& Gupta (2004) suggested that this species is an indicator of intermediate to high sustained flux of organic matter to the sea floor in the southeastern Indian Ocean.

Globocassidulina subglobosa is a cosmopolitan species occurring over a wide range of bathymetry with different water masses, largely reflecting well-oxygenated deep waters with strongly pulsed food supply and good carbonate preservation in commonly oligotrophic environments (Singh \& Gupta, 2004) in the southeastern Indian Ocean. Fariduddin \& Loubere (1997) observed this species associated with NADW in the Atlantic Ocean and categorized it as a low productivity species, whereas Corliss (1979) found it associated with AABW in the southwest Indian Ocean. This taxon is often found abundant in sediments receiving less organic matter in regions where strong bottom currents are likely to occur (Schmiedl et al., 1997; Nees \& Struck, 1999). Ohkushi et al. (2000) suggest that Globocassidulina lives in areas of enhanced and continuous food supply. In the south Atlantic, high abundances of G. subglobosa are found within the depth range of Circumpolar Deep Water (Schnitker, 1980) and in oligotrophic areas at higher elevations of ridges and submarine hills (Mackensen et al., 1995). Gooday (1994) suggested that $G$. subglobosa feeds on phytodetritus, reflecting pulsed food supply to the ocean floor. This species is thus 


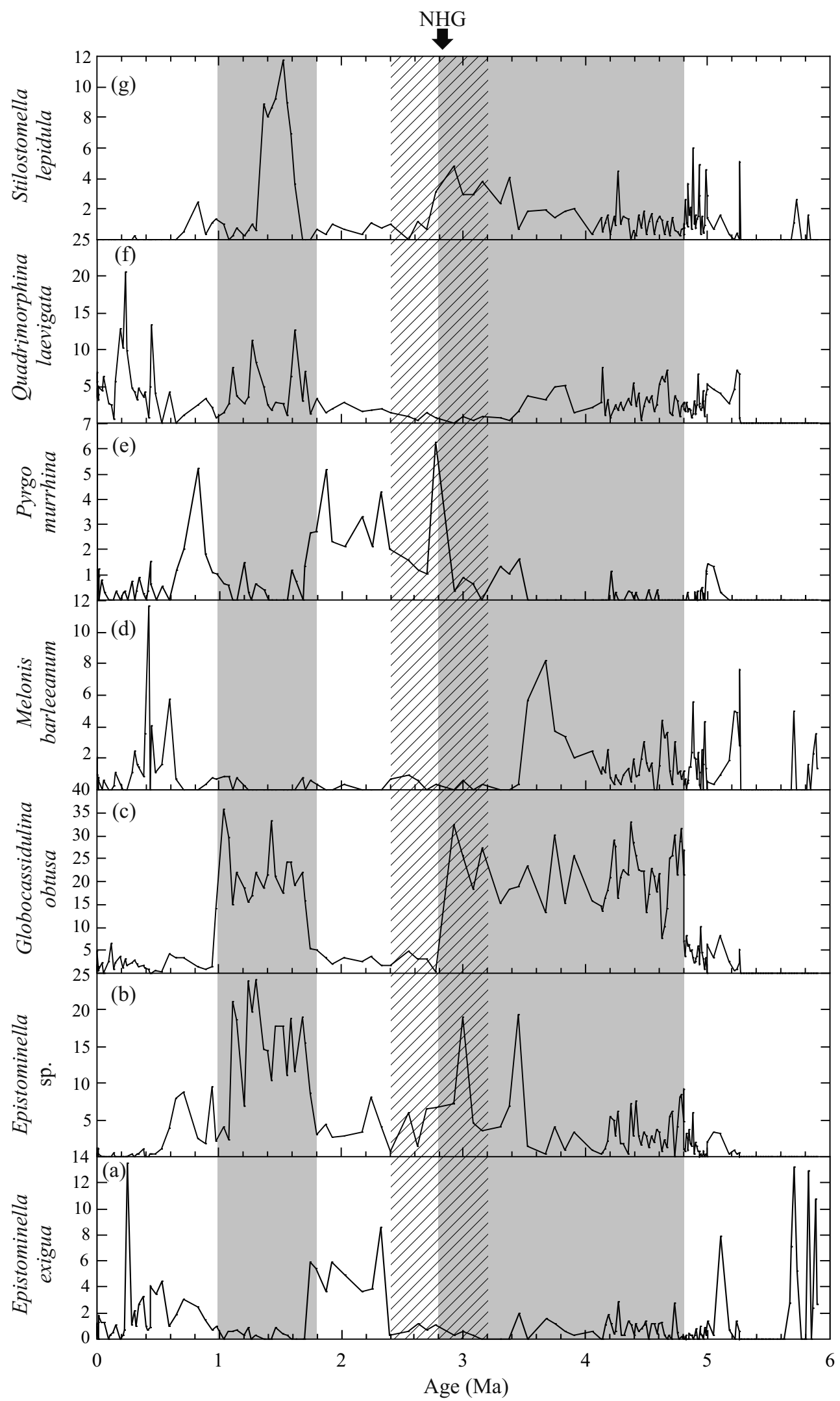

Fig. 3. Percent distribution of important species of benthic foraminifera at ODP hole 991A, Blake Ridge during the past 5.9 Ma. Grey bars indicate intervals interpreted as indicative for increased transport of organic-rich sediments to the Blake Ridge by the Deep Western Boundary Undercurrent (DWBUC). Hatched area indicates beginning of the major intensification of the Northern Hemisphere glaciation (NHG) (Zachos et al., 2001). 


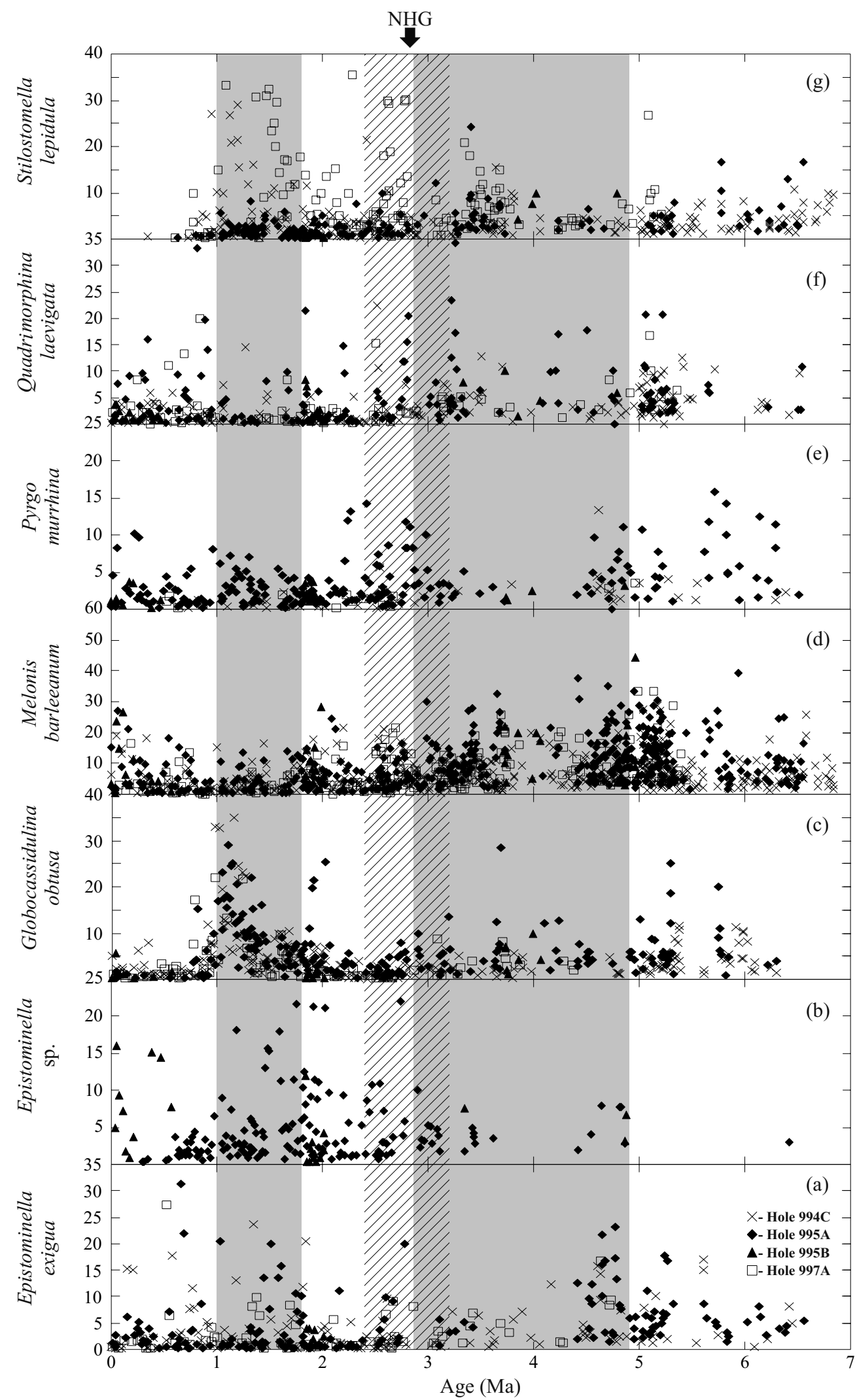

Fig. 4. Percent distribution of important species of benthic foraminifera at ODP holes 994C, 995A and B, and 997A, Blake Ridge during the past 6.8 Ma. For grey bars and hatched area, see Fig. 3. 


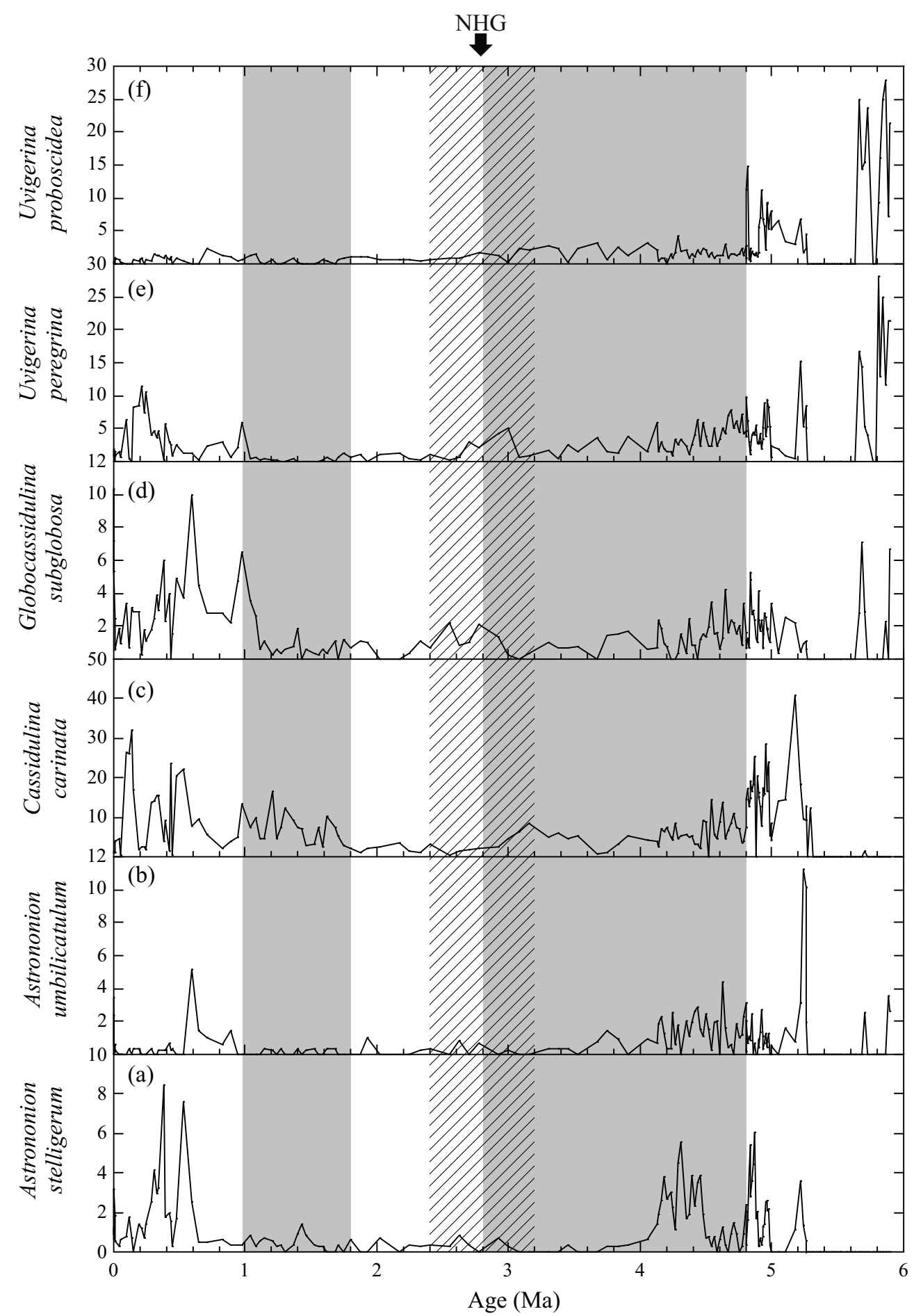

Fig. 5. Percent distribution of important species of benthic foraminifera at ODP hole 991A, Blake Ridge during the past 5.9 Ma. For grey bars and hatched area, see Fig. 3.

suggestive of NADW and appears to be an opportunist with great powers of adaptation.

High abundances of Melonis barleeanum in the north Atlantic are characteristic of high productivity regions with sustained flux of organic matter to the sea floor (Thomas et al., 1995). Extraordinary high abundance of this species in the eastern south Atlantic indicates a strong supply of organic matter to the sea floor (Schmiedl \& Mackensen, 1997). Evenly distributed pores on the test of infaunal species Melonis barleeanum suggest an adaptation to gas exchange in low oxygen conditions (Corliss, 1985; Fontanier et al., 2005) and during high food supply (Arnold, 1983; Caralp, 1988). In the Indian Ocean, M. barleeanum indicates intermediate organic flux with intermediate to high seasonality and the presence of degraded 


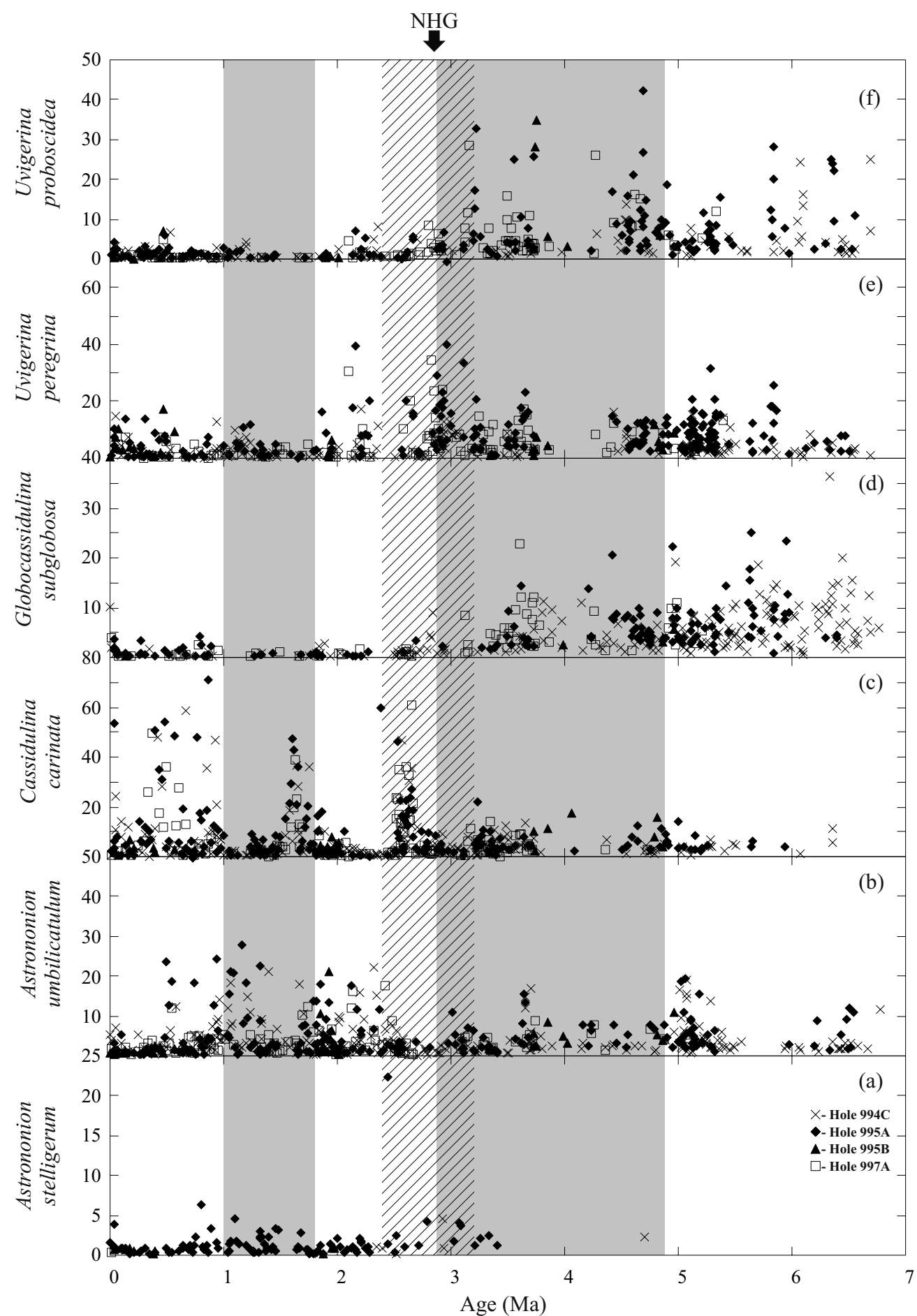

Fig. 6. Percent distribution of important species of benthic foraminifera at ODP holes 994C, 995A and B, and 997A, Blake Ridge during the past 6.8 Ma. For grey bars and hatched area, see Fig. 3.

organic matter (Gupta \& Thomas, 2003). It has also been reported as high productivity taxon from the Pacific Ocean (Loubere, 1991, 1994). In hydrocarbon-seep environments, $M$. barleeanum is common due to the availability of bacteria as the source of food (Boetius et al., 2000; Panieri, 2005).

Pyrgo murrhina lives epifaunally (Corliss \& Chen, 1988) in low organic carbon environments (Lutze \& Coulbourn, 1984), preferring cool, well-ventilated waters (Caralp, 1984; Gupta \& Srinivasan, 1996; Gupta \& Thomas, 2003). Gupta \& Satapathy (2000) suggested it is a species preferring cool, oxygenated waters with low organic food, whereas Gupta \& Thomas (1999) described $P$. murrhina as preferring high oxygen waters with pulsed food supply and good carbonate preservation. This species has been observed in a deep environment in the eastern 


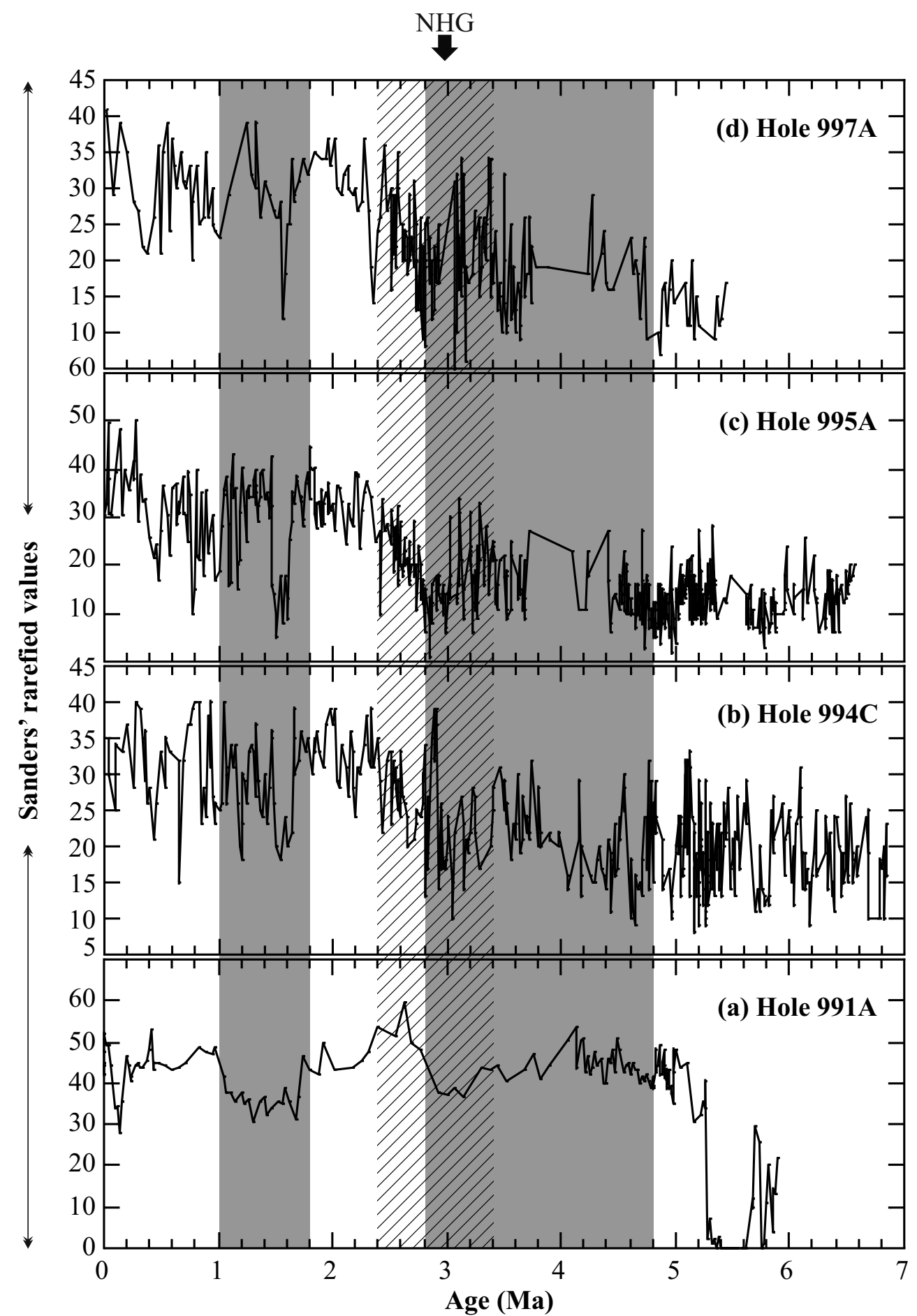

Fig. 7. Sanders' rarefied values indicating changes in species diversity since the late Miocene at ODP holes 991A, 994C, 995A and 997A, Blake Ridge. Values were calculated using the method described in Singh \& Gupta (2004). A major increase at $c$. 3 Ma in holes 994C, 995A and 997A coincides with the beginning of major Northern Hemisphere glaciation. For grey bars and hatched area, see Fig. 3.

Indian Ocean at temperatures $>2.5^{\circ} \mathrm{C}$ and oxygen $>3.5 \mathrm{ml} \mathrm{l}^{-1}$ (Murgese \& De Deckker, 2005).

Stilostomella lepidula is a cosmopolitan taxon, which reflects complicated ecological preferences that are yet to be fully understood. Boersma (1990) suggested that $S$. lepidula prefers to live in sediments that are organic carbon rich and moderately low in oxygen. In the present study, S. lepidula disappears across the mid-Pleistocene Transition (MPT) which has also been observed in earlier studies (Gupta, 1993; Hayward, 2001; Kawagata et al., 2006). The extinction of this species across the MPT has further complicated our understanding of its ecological preference. 
The environmental preferences of Quadrimorphina laevigata are not well constrained. This species has been found associated with Bolivina paula, Bulimina striata, Cassidulina laevigata, Epistominella exigua and Globocassidulina obtusa, which are suggestive of high organic carbon and low oxygen environments.

The genus Uvigerina is commonly known as a high productivity taxon (Loubere, 1991, 1994; Rathburn \& Corliss, 1994). The shallow infaunal Uvigerina peregrina is closely related to continuous organic carbon flux irrespective of oxygen levels (Miller \& Lohmann, 1982; Rathburn \& Corliss, 1994; Mackensen et al., 1995). In a recent study, De \& Gupta (2010) observed the dominance of Uvigerina peregrina in the oxygen minimum zone (OMZ) of the northwest Arabian Sea, where surface productivity is highest and oxygen is at a minimum. It was also reported as a dominant species from below the OMZ by previous workers, from the northwest (Hermelin \& Shimmield, 1990), as well as the northeast (Jannink et al., 1998; Maas, 2000; Schumacher et al., 2007), Arabian Sea where there is high flux of organic matter (Jannink et al., 1998; Schumacher et al., 2007). Altenbach \& Sarnthein (1989) and Fontanier et al. (2002) suggested that $U$. peregrina prefers a microhabitat rich in bacteria, exoenzymes and meiofauna, and is typical of sediments enriched in organic carbon and depleted in oxygen, which is common in areas below upwelling productivity zones. Fontanier et al. (2002) and Geslin et al. (2004) argued that this species has a variable response to varying oxygen conditions. In a recent study, this species was observed to tolerate in situ a temperature range of $10.6-13.9^{\circ} \mathrm{C}$, oxygen $0.25-0.56 \mathrm{ml} \mathrm{l}^{-1}$ and a large range of primary production (De \& Gupta, 2010).

Uvigerina proboscidea blooms in high productivity regions of the Indian Ocean (Gupta \& Srinivasan, 1992; Gupta \& Thomas, 1999; Almogi-Labin et al., 2000), particularly when productivity is high throughout the year and seasonality of food supply is low or absent (Loubere, 1998; Gupta \& Thomas, 1999; Loubere \& Fariduddin, 1999; Ohkushi et al., 2000). This species is correlated positively with organic carbon flux and negatively with dissolved-oxygen concentration in the eastern Indian Ocean (Murgese \& De Deckker, 2005). Uvigerina proboscidea was reported by Gupta (1994) from 1200-5000 m water depth in the Recent sediments of the Indian Ocean, with the highest abundance between $1700 \mathrm{~m}$ and $2300 \mathrm{~m}$ in the eastern sector. Peaks of $U$. proboscidea abundances are inferred to represent times of high surface productivity related to intense trade winds during the SW Indian monsoon causing widespread upwelling along equatorial divergence in the Indian Ocean (Gupta \& Srinivasan, 1992). In Recent sediments of the Indian Ocean, U. proboscidea is found in conditions of in situ temperature of $2.1-9.5^{\circ} \mathrm{C}$, oxygen from $0.96-3.29 \mathrm{ml} \mathrm{l}^{-1}$ and high phosphate and nitrate concentrations (De \& Gupta, 2010).

\section{DISCUSSION}

Recent studies suggest that, in general, benthic foraminiferal distribution is limited by a combination of food availability and oxygenation (Sen Gupta \& Machain-Castillo, 1993; Jorissen et al., 1995; Gooday, 2003). However, in areas where the oxygen content of bottom waters is not a limiting factor, the amount of organic flux to the sea floor mainly governs the occurrence of benthic species in the sediments (Van der Zwaan et al., 1999; Friedrich \& Hemleben, 2007).
Benthic foraminiferal faunas from sites 991, 994, 995 and 997 suggest significant palaeoceanographic changes in the Blake Ridge area during the late Miocene to the Pleistocene. We did not observe any species or species assemblage endemic to Blake Ridge methane settings, which supports the earlier findings of Panieri \& Sen Gupta (2008) and Lobegeier \& Sen Gupta (2008), for example, and thus we interpret benthic faunal data from this region in terms of organic food supplied by the DWBUC, linked to the intensity of NADW or NCW. Since hole 991A lies on a diapir, having both disturbed and undisturbed sediment column, we interpret faunal data from this hole cautiously. At hole 991A, the older interval (6-3 Ma), covering the gas hydrate zone, may have suffered some disturbance in the sediment column, whereas the younger interval appears to be undisturbed. Benthic faunas (e.g. G. obtusa, M. barleeanum) at hole 991A suggest high organic flux during 4.8-2.8 Ma, which corresponds to the early Pliocene warm period preceding major NHG. The high organic carbon during the early Pliocene warmth resulted from increased transport of terrigenous matter rich in refractory organic material from the continental margin of Canada by the DWBUC in response to increased production of the NADW (Laine et al., 1994; Balsam \& Damuth, 2000). It has been widely suggested that NADW flow increases during warm intervals and decreases during colder periods (Raymo et al., 1990, 1996; Kim \& Crowley, 2000). At sites 994, 995 and 997, the early Pliocene interval is marked by higher abundances of G. subglobosa, M. barleeanum, $U$. peregrina and $U$. proboscidea, indicating increased availability of organic carbon. These species corroborate that during warm intervals there was increased transport of organic-rich sediments by the NADW-driven DWBUC to the Blake Ridge. Although there is not a one-to-one correlation between benthic data from site 991 and combined data from sites 994, 995 and 997, the overall trends are similar between the two areas.

From 2.8 to $1.8 \mathrm{Ma}$, the G. obtusa population decreases, whereas $P$. murrhina and E. exigua show an increase at all the studied sites (Figs 3, 4), indicating oligotrophic and welloxygenated conditions at the Blake Ridge. This was an interval of intensified NHG and decreased production of the NADW, resulting in weakening of the DWBUC and apparent decreased transport of nutrients to the Blake Ridge. An increase in species diversity (Sanders' rarefied values) at $3 \mathrm{Ma}$ probably resulted from a decreased population of bacteria due to low organic matter and/or less competition. Singh \& Gupta (2005) observed a similar relationship between benthic foraminifera and bacteria-rich organic carbon in the eastern Indian Ocean ODP hole 757B. An abrupt increase in G. obtusa and decrease in $P$. murrhina at hole 991A during 1.8-1.0 Ma indicates another pulse of increased transport of organic-rich sediments to the Blake Ridge. However, such a faunal pattern does not exist at holes 994C, 995A, B and 997A, where the species C. carinata and S. lepidula show episodic occurrences (Fig. 6), although Sanders' values show a short-lived decrease during 1.8-1 Ma (Fig. 7). We argue that these faunal trends indicate decreased delivery of organic-rich sediments by DWBUC to the Blake Ridge region following $\mathrm{NHG}$, which may have benefited the opportunistic benthic species. The shipboard scientific data suggest decreased total organic carbon and sediment accumulation rates, and increased calcium carbonate $\left(\mathrm{wt}^{\mathrm{O}} \%\right)$ in the late 
Pliocene (c. 3 Ma) at Blake Ridge ODP holes (Paull et al., 1996). Thus, our observations strengthen a link between fauna and sediment characteristics at Blake Ridge.

In the late Pleistocene (c.0.6 Ma), Stilostomella lepidula became extinct in all the holes studied (Figs 3-4). This species became extinct globally during the late Pleistocene cooling of the deep sea (Kawagata et al., 2005; Hayward et al., 2010). Stilostomella lepidula has complexly structured apertures, suggesting that it may have possessed a mode of feeding that no longer exists in the cold, well-ventilated oceans of the present (Hayward et al., 2010).

\section{CONCLUSIONS}

This study provides useful information about the transport of organic carbon-rich sediments by the Deep Western Boundary Undercurrent (DWBUC) to the studied sites, which provided a food source to benthic foraminifera in the Blake Ridge region. The sediment delivery to the Blake Ridge was closely related to the intensity of the DWBUC driven by production of the North Atlantic Deep Water (NADW). The enhanced population of uvigerinids in the late Miocene suggests an increased influence of the Southern Component Water (SCW). During the Pliocene warm interval (4.8-2.8 Ma) the intensity of DWBUC increased and enhanced the supply of terrestrial organic carbon to the Blake Ridge. The interval between 2.8 and $1.8 \mathrm{Ma}$ coincides with the initiation of Northern Hemisphere glaciation (NHG), during which the production of NADW decreased and oligotrophic conditions prevailed. During 1.8-1 Ma, the faunal trends indicate decreased delivery of organic-rich sediments by DWBUC to the Blake Ridge region following the NHG. During the mid-Pleistocene Transition (c. 0.6 Ma), Stilostomella lepidula became extinct, which has been documented in the global ocean and related to the cooling of the deep sea.

\section{ACKNOWLEGEMENTS}

AKG thanks the Ocean Drilling Program (ODP) for providing core samples (under ODP request no. 16030A, 16030B and $16030 \mathrm{C}$ ) for the present study. KM was supported by the Indian Institute of Technology, Kharagpur fellowship. The SEM Laboratories of the Department of Geology \& Geophysics and Central Research Facility, IIT Kharagpur are gratefully acknowledged for taking the micrographs. Constructive reviews by two anonymous reviewers and the handling Editor are acknowledged with thanks.

\section{APPENDIX A: SYSTEMATIC DESCRIPTIONS OF THE SPECIES RECORDED FROM THE STUDY AREA, WITH ORIGINAL REFERENCES}

Genus Alabaminella Saidova, 1975

Alabaminella weddellensis (Earland, 1950)

(P1. 1, figs 1-2)

1950 Eilohedra pusilla Parr: 14, fig. 16a-c.

Occurrence. Late Miocene-Holocene; ODP holes 991A, 994C, 997A.
Genus Anomalina d'Orbigny, 1826

Anomalina globulosa (Chapman \& Parr, 1937)

(Pl. 1, fig. 3)

1937 Anomalina globulosa Chapman \& Parr: 117, pl. 9, fig. 27.

Occurrence. Late Miocene-Holocene; ODP holes 991A, 994C, 995A, 995B, 997A.

Genus Astacolus de Montfort, 1808

Astacolus reniformis (d'Orbigny, 1846)

(Pl. 1, fig. 4)

1846 Cristellaria reniformis d'Orbigny: 88, pl. 3, figs 39-40.

Occurrence. Late Pliocene-Middle Pleistocene; ODP holes 994C, 995A, 995B.

Genus Astrononion Cushman \& Edwards, 1937

Astrononion stelligerum (d'Orbigny, 1839)

(Pl. 1, figs 5-6)

1839 Nonionina stelligera d'Orbigny: 128, pl. 3, figs 1-2.

Occurrence. Late Pliocene-Holocene; ODP holes 991A, 994C, 995A, 995B, 997A.

Astrononion umbilicatulum (Uchio, 1952)

(Pl. 1, figs 7-9)

1952 Astrononion umbilicatulum Uchio: 36, fig. 1.

Occurrence. Late Miocene-Holocene; ODP holes 991A, 994C, 995A, 995B, 997A.

Genus Awhea Vella, 1963

Awhea tosta (Schwager, 1866)

(P1. 1, fig. 10)

1866 Nodosaria tosta Schwager: 219, pl. 5, fig. 42.

Occurrence. Late Miocene-Late Pliocene; ODP holes 991A, 994C, 995A, 995B, 997A.

Genus Bolivina d'Orbigny, 1839

Bolivina alata (Seguenza, 1862)

(P1. 1, figs 11-12)

1862 Valvulina alata Seguenza: 115, pl. 2, figs 5-5a.

Occurrence. Late Pliocene-Middle Pleistocene; ODP hole 991A.

Bolivina paula (Cushman \& Cahill, 1932)

(P1. 1, figs 13-14) 


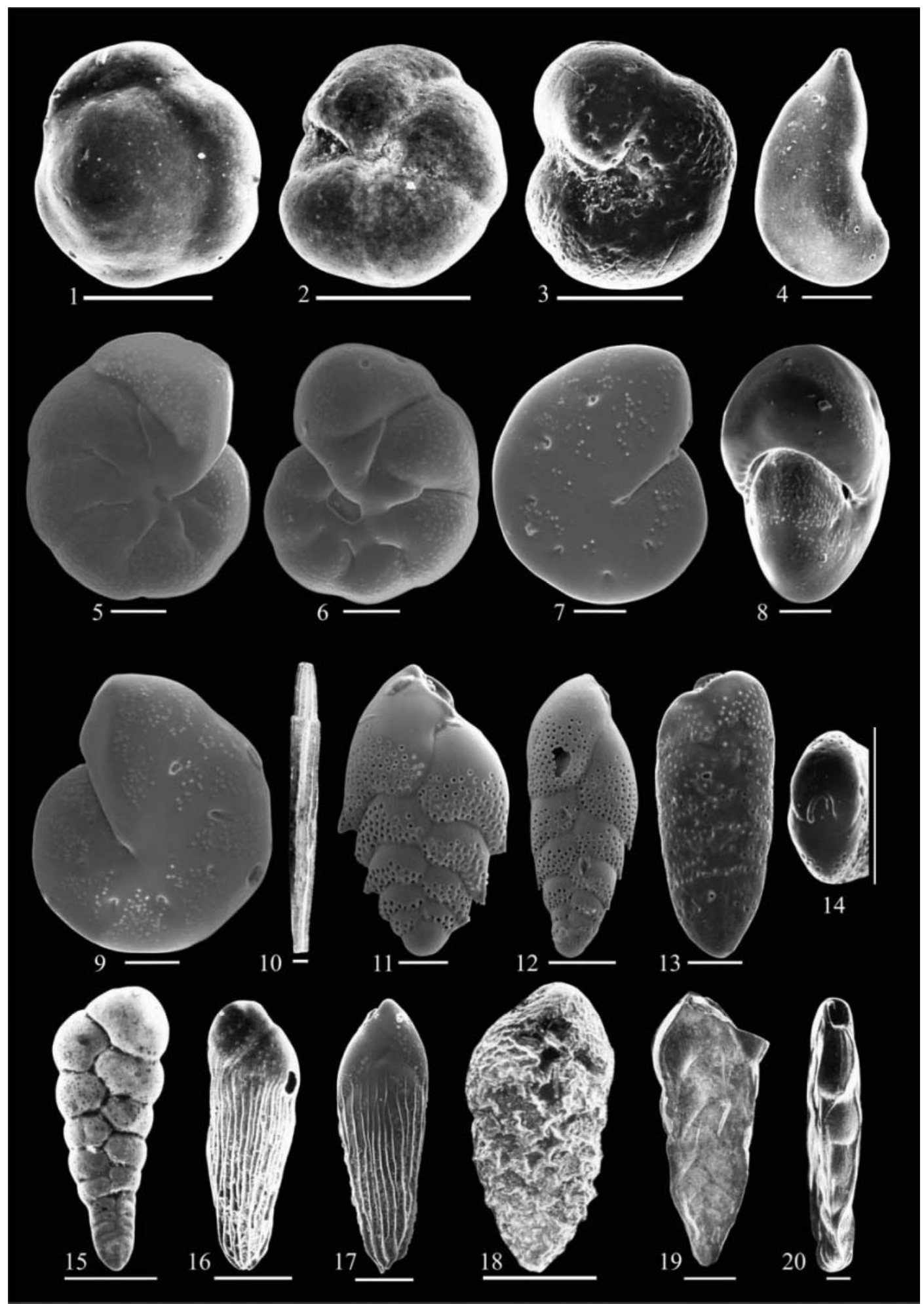

Explanation of Plate 1.

figs 1, 2. Alabaminella weddellensis, 164-994C, 17H-CC, 0-2: 1, spiral view; 2, umbilical view. fig. 3. Anomalina globulosa, 164-994C, 27P-1, 0-2, umbilical view. fig. 4. Astacolus reniformis, 164-994C, 55X-3, 32-24, side view. figs 5, 6. Astrononion stelligerum, 164-995A, 5H-6, 148-150, side view. figs 7-9. Astrononion umbilicatulum, 164-995A, 5H-3, 148-150: 7, 9, side view; 8, apertural view. fig. 10. Awhea tosta, 164-994C, 35X-CC, 0-2, side view. figs 11, 12. Bolivina alata, 164-991A, 3H-7, 25-27; side view. figs 13, 14. Bolivina paula, 164-991A, 1H-1, 25-27: 13, side view; 14, apertural view. fig. 15. Bolivina pseudopunctata, 164-994C, 41X-4, 70-72, side view. figs 16, 17. Bolivina pusilla: 16, 164-994C, 30X-CC, 50-52, side view; 17, 164-995A, 5H-6, 148-150, side view. fig. 18. Bolivina thalmanni, 164-994C, 76X-1, 74-76, side view. figs 19, 20. Bolivinita quadrilatera, 164-994C: 19, 41X-6, 70-72, side view; 20, 5H-3, 75-77, apertural view. Scale bar $50 \mu \mathrm{m}$ for figs $5-9,11,13 ; 100 \mu \mathrm{m}$ for the rest. 
1932 Bolivina paula (Cushman \& Cahill); Cushman \& Ponton: 84, pl. 12 , fig. $6 a-$ b.

Occurrence. Late Miocene-Holocene; ODP holes 991A, 994C, 995A, 995B, 997A.

Bolivina pseudopunctata (Hoeglund, 1947)

(Pl. 1, fig. 15)

1947 Bolivina pseudopunctata Hoeglund: 273, pl. 24, fig. 5; pl. 31 figs 23-24.

Occurrence. Late Miocene-Holocene; ODP holes 991A, 994C, 995A, 995B, 997A.

Bolivina pusilla (Schwager, 1866)

(Pl. 1, figs 16-17)

1866 Bolivina pusilla Schwager: 254, pl. 7, fig. 101.

Occurrence. Late Pliocene-Middle pleistocene; ODP holes 991A, 994C, 995A, 995B.

Bolivina thalmanni (Renz, 1948)

(P1. 1, fig. 18)

1948 Bolivina thalmanni Renz: 120, pl. 12, fig. 13.

Occurrence. Late Pliocene-Middle Pleistocene; ODP hole 994C.

Genus Bolivinita Cushman, 1927

Bolivinita quadrilatera (Schwager, 1866)

(P1. 1, figs 19-20)

1866 Textilaria quadrilatera Schwager: 253, pl. 7, fig. 103.

Occurrence. Late Pliocene-Middle Pleistocene, ODP holes 991A, 994C, 995A, 995B, 997A.

Genus Bulimina d'Orbigny, 1826

Bulimina aculeata (d'Orbigny, 1826)

(P1. 2, figs 1-2)

1826 Bulimina aculeata d'Orbigny: 269, pl. 12, figs 10-12.

Occurrence. Late Miocene-Holocene; ODP holes 991A, 994C, 995A, 995B, 997A.

Bulimina alazanensis (Cushman, 1927)

(Pl. 2, figs 3-4)

1927 Bulimina alazanensis Cushman: 161, pl. 25, fig. 4.

Occurrence. Late Miocene-Holocene; ODP holes 991A, 994C, 995A, 995B, 997A.

Bulimina marginata (d'Orbigny, 1826)

(P1. 2, fig. 5)
1826 Bulimina marginata d'Orbigny: 269, pl. 12, figs 10-12.

Occurrence. Late Miocene-Holocene; ODP holes 991A, 994C, 995A, 995B, 997A.

Bulimina rostrata ((Brady, 1884)

(P1. 2, fig. 6)

1884 Bulimina rostrata Brady: 408, pl. 5, figs 14-15.

Occurrence. Early Pliocene; ODP hole 994C.

Bulimina striata (d'Orbigny, 1826)

(Pl. 2, figs 7-8)

1826 Bulimina striata d'Orbigny: 269, no. 2.

Occurrence. Late Miocene-Holocene; ODP holes 991A, 995A, 995B, 997A.

Bulimina translucens (Parker, 1958)

(Pl. 2, fig. 9)

1953 Bulimina translucens Parker: 33, pl. 6, figs 30-31.

Occurrence. Middle Pliocene-Late Pleistocene; ODP holes 994C, 997A.

Genus Buliminella Cushman, 1911

Buliminella elegantissima (d'Orbigny, 1839)

(Pl. 2, fig. 10)

1839 Bulimina elegantissima d'Orbigny: 51, pl. 7, figs 13-14.

Occurrence. Late Miocene-Holocene; ODP holes 991A, 994C, 995A, 995B, 997A.

Genus Cassidulina d'Orbigny, 1826

Cassidulina carinata (Silvestri, 1896)

(Pl. 2, figs 11-13)

1896 Cassidulina laevigata var. carinata Silvestri: 104, pl. 2, fig. 10 .

Occurrence. Late Miocene-Holocene, ODP holes 991A, 994C, 995A, 995B, 997A.

Cassidulina crassa (d'Orbigny, 1839)

(Pl. 2, fig. 14)

1839 Cassidulina crassa d'Orbigny: 56, pl. 7, figs 18-20.

Occurrence. Middle Pliocene-Early Pleistocene, ODP holes 991A, 994C.

Cassidulina laevigata (d'Orbigny, 1826)

(P1. 2, figs 15-16) 


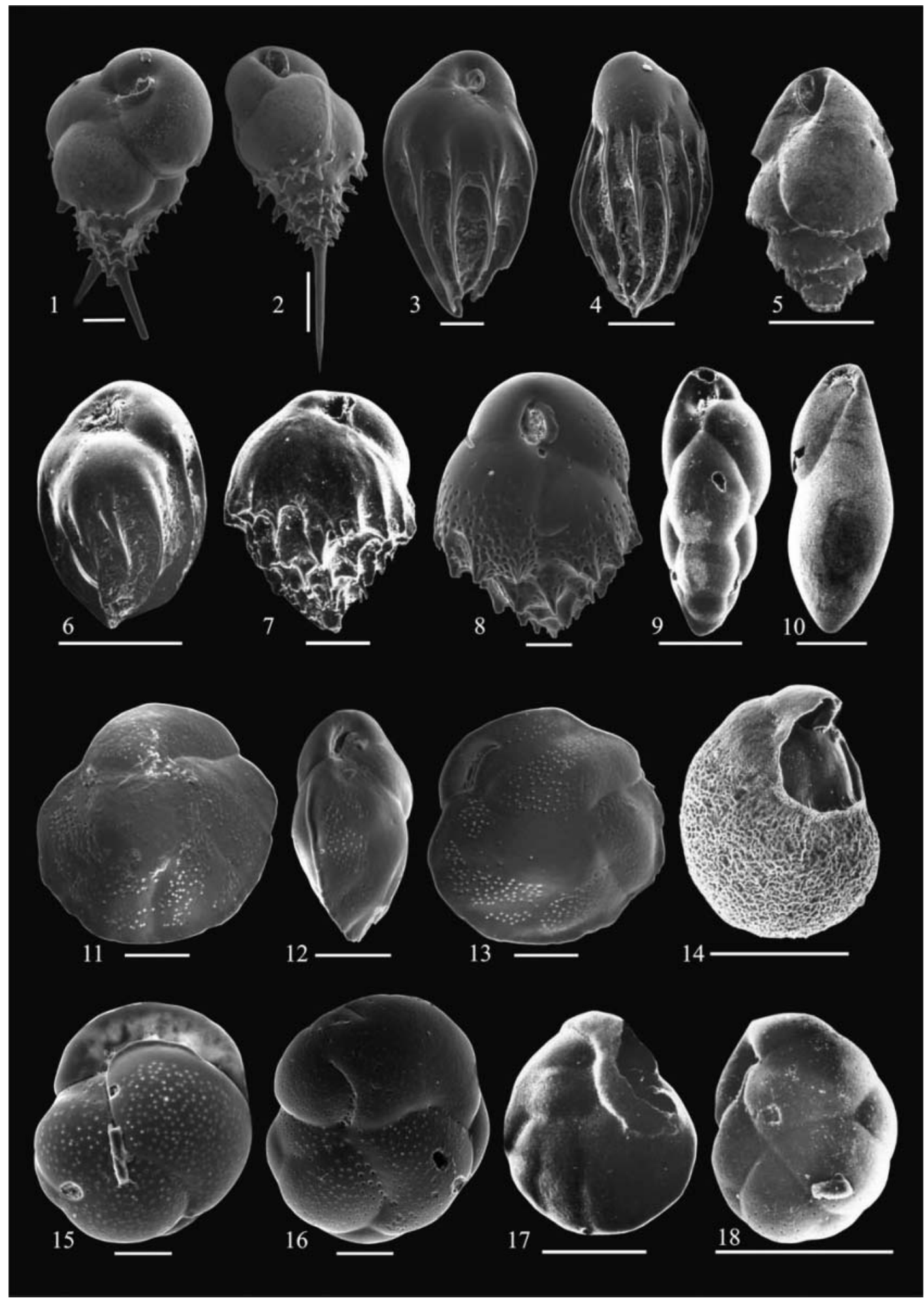

Explanation of Plate 2.

figs 1, 2. Bulimina aculeata, 164-995A, 4H-6, 148-150: 1, side view; 2, apertural view. figs 3, 4. Bulimina alazanensis, 164-995A, 5H-4, 148-150: 3, front view; 4, side view. fig. 5. Bulimina marginata, 164-994C, 5H-7, 30-32, front view. fig. 6. Bulimina rostrata, 164-994C, 58X-CC, 0-2, front view. figs 7, 8. Bulimina striata: 7, 164-994C, 30X-3, 69-71, side view; 8, 164-995A, 10H-7, 75-77, apertural view. fig. 9. Bulimina translucens, 164-994C, 21X-2, 75-77, front view. fig. 10. Buliminella elegantissima, 164-994C, 17H-1, 74-76, front view. figs 11-13. Cassidulina carinata, 164-995A, 6H-2, 75-77: 11, spiral view; 12, apertural view; 13, umbilical view. fig. 14. Cassidulina crassa, 164-994C, 54X-3, 72-74, umbilical view. figs 15, 16. Cassidulina laevigata, 164-991A, 1H-1, 25-27: 15, umbilical view; 16, spiral view. fig 17. Cassidulina limbata, 164-994C, 16H-1, 75-77, umbilical view. fig. 18. Cassidulina teretis, 164-994C, 34X-1, 4-6, umbilical view. Scale bar $50 \mu \mathrm{m}$ for figs $1,3,9,15-16$; $100 \mu \mathrm{m}$ for the rest. 
1826 Cassidulina laevigata d’Orbigny: 282, pl. 15, figs 4-5.

Occurrence. Late Miocene-Holocene; ODP holes 991A, 994C, 995A, 995B, 997A.

Cassidulina limbata (Cushman \& Hughes, 1925)

(Pl. 2, fig. 17)

1925 Cassidulina limbata Cushman \& Hughes: 12, pl. 2, fig. 2.

Occurrence. Middle Pliocene-Late Pliocene; ODP hole 994C.

Cassidulina teretis (Tappan, 1951)

(Pl. 2, fig. 18)

1951 Cassidulina teretis Tappan: 7, pl. 1, fig. 30.

Occurrence. Early Pliocene; ODP hole 994C.

Genus Chilostomella Reuss, 1849

Chilostomella oolina (Schwager, 1878)

(Pl. 3, figs 1-2)

1878 Chilostomella oolina Schwager: 527, pl. 1, fig. 16.

Occurrence. Late Miocene-Holocene; ODP holes 991A, 994C, 995A, 995B.

Genus Chrysalogonium Schubert, 1907

Chrysalogonium deceptoria (Schwager, 1866)

(Pl. 3, figs 3-4)

1866 Nodosaria deceptoria Schwager: 231, pl. 6, fig. 66.

Occurrence. Late Miocene-Early Pleistocene; ODP hole 995A.

Chrysalogonium gomphiformis (Schwager, 1866)

(P1. 3, fig. 5)

1866 Nodosaria gomphiformis Schwager: 220, pl. 5, fig. 48.

Occurrence. Middle Pliocene-Early Pleistocene; ODP hole 994C, 995A, 997A.

Chrysalogonium intertenuatum (Schwager, 1866)

(P1. 3, fig. 6)

1866 Nodosaria intertenuata Schwager: 226, pl. 6, fig. 58.

Occurrence. Late Miocene-Middle Pleistocene; ODP hole 991A, 994C, 995A, 997A.

Genus Cibicides de Montfort, 1808

Cibicides bradyi (Trauth, 1918)

(P1. 3, figs 7-9)

1918 Truncatulina bradyi Trauth: 235, pl. 4, figs 7-9.

Occurrence. Late Miocene-Holocene; ODP holes 991A, 994C, 995A, 995B, 997A.

Cibicides kullenbergi (Parker, 1953)

(P1. 3, figs 10-12)

1953 Cibicides kullenbergi (Parker); Phleger, Parker \& Peirson: 49, pl. 11, figs 7-8.

Occurrence. Late Miocene-Holocene; ODP holes 991A, 994C, 995A, 995B, 997A.

Cibicides wuellerstorfi (Schwager, 1866)

(Pl. 3, figs 13-15)

1866 Anomalina wuellerstorfi Schwager: 258, pl. 7, fig. 105.

Occurrence. Late Miocene-Holocene; ODP holes 991A, 994C, 995A, 995B, 997A.

Genus Cornuspira Schultze, 1854

Cornuspira carinata (Costa, 1856)

(Pl. 3, fig. 16)

1856 Operculina carinata Costa: 209, pl. 17, fig. 15.

Occurrence. Late Miocene-Holocene; ODP holes 994C, 997A.

Cornuspira involvens (Reuss, 1850)

(Pl. 3, fig. 17)

1850 Operculina involvens Reuss: 370, pl. 45, fig. 20.

Occurrence. Middle Pliocene-Holocene; ODP holes 995A, 997A.

\section{Genus Cymbaloporetta Cushman 1928 \\ Cymbaloporetta bradyi (Cushman, 1915)}

(P1. 3, fig. 18)

1915 Cymbalopora poeyi (d'Orbigny) var. bradyi Cushman: 10, fig. $2 \mathrm{a}-\mathrm{c} ;$ pl. 14, fig. $2 \mathrm{a}-\mathrm{c}$.

Occurrence. Late Pliocene-Middle Pleistocene; ODP holes 994C, 997A.

Genus Dentalina Risso, 1826

Dentalina neugeboreni (Schwager, 1866)

(P1. 3, figs 19-20)

1866 Nodosaria neugeboreni Schwager: 232, pl. 6, fig. 67.

Occurrence. Late Miocene-Holocene; ODP holes 991A, 994C, 995A, 995B. 


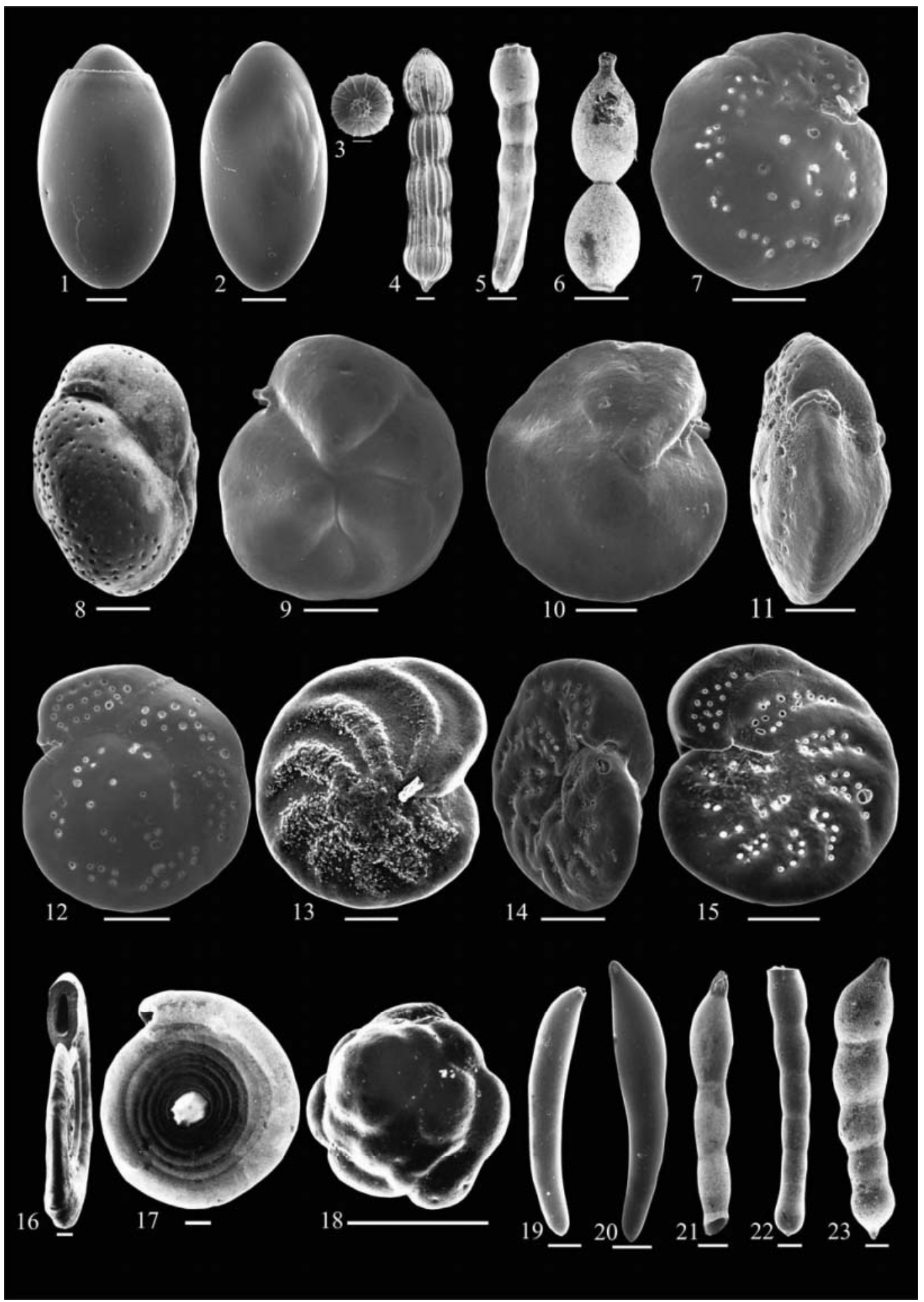

Explanation of Plate 3.

figs 1, 2. Chilostomella oolina, 164-991A, 6H-2, 55-57, side view. figs 3, 4. Chrysalogonium deceptoria: 3, 164-997A, 55P-1, 79-81, apertural view; 4, 164-994C, 44X-2, 72-74, side view. fig. 5. Chrysalogonium gomphiformis, 164-994C, 21X-4, 75-77, side view. fig. 6. Chrysalogonium intertenuatum, 164-994C, 33X-3, 69-71, side view, figs 7-9. Cibicides bradyi: 7, 164-991A, 1H-1, 25-27, spiral view; 8, 164-994C, 41X-3, 75-77, apertural view; 9, 164-991A, 4H-6, 148-150, umbilical view. figs 10-12. Cibicides kullenbergi: 10, 164-995A, 10H-5, 148-150, umbilical view; 11, 164-991A, 5H-5, 147-149, apertural view; 12, 164-995A, 10H-5, 148-150, spiral view. figs 13-15. Cibicides wuellerstorfi, 164-995A, 10H-5, 10H-5, 148-150: 13, umbilical view; 14, apertural view; 15, spiral view. fig. 16. Cornuspira carinata, 164-994C, 84X-3, 71-73, apertural view. fig. 17. Cornuspira involvens, 164-994C, 1H-1, 0-2, side view. fig. 18. Cymbaloporetta bradyi, 164-994C, 15H-7, 75-77, spiral view. figs 19, 20. Dentalina neugeboreni: 19, 164-994C, 34X-1, 4-6, side view; 20, 164-995A, 4H-6, 148-150, side view. figs 21, 22. Dentalina stimulea, 164-994C: 21, 35X-CC, 0-2, side view; 22, 19H-1, 0-2, side view. fig. 23. Dentalina subsoluta, 164-994C, 22X-4, 70-72, side view. Scale bar $50 \mu \mathrm{m}$ for $13 ; 100 \mu \mathrm{m}$ for the rest. 
Dentalina stimulea (Schwager, 1866)

(Pl. 3, figs 21-22)

1866 Nodosaria stimulea Schwager: 226, pl. 6, fig. 57.

Occurrence. Late Miocene-Middle Pleistocene; ODP holes 991A, 994C, 995A, 997A.

Dentalina subsoluta (Cushman, 1923)

(P1. 3, fig. 23)

1923 Nodosaria subsoluta Cushman: 74, pl. 13, fig. 1.

Occurrence. Late Miocene-Late Pleistocene; ODP holes 991A, 994C, 995A, 995B, 997A.

Genus Discopulvinulina Hofker, 1951

Discopulvinulina bertheloti (d'Orbigny, 1839)

(P1. 4, fig. 1)

1839 Rosalina bertheloti d'Orbigny: 135, pl. 1, figs 28-30.

Occurrence. Late Miocene-Holocene; ODP holes 991A, 994C, 995A, 995B, 997A.

Genus Dorothia Plummer, 1931

Dorothia brevis (Cushman \& Stainforth, 1945)

$$
\text { (P1. 4, fig. 2) }
$$

1945 Dorothia brevis Cushman \& Stainforth: 18, pl. 2, fig. 5.

Occurrence. Early Pliocene-Holocene; ODP holes 994C, 997A.

Genus Eggerella Cushman, 1935

Eggerella bradyi (Cushman, 1911)

(P1. 4, figs 3-4)

1911 Verneuilina bradyi Cushman: 54, pl. 2, fig. 87a-b.

Occurrence. Late Miocene-Holocene; ODP holes 991A, 994C, 995A, 995B, 997A.

Genus Ellipsobulimina Silvestri, 1903

Ellipsobulimina seguenzai (Silvestr, 1903)

(P1. 4, fig. 5)

1903 Ellipsobulimina seguenzai Silvestri: 14, 15, figs 1-7.

Occurrence. Early Pliocene; ODP hole 994C.

$$
\text { Ellipsobulimina sp. }
$$

(P1. 4, fig. 6)

Occurrence. Early Pliocene; ODP hole 994C.
Remarks. Not enough specimens found to assign any species name.

Genus Ellipsoidella Heron-Allen \& Earland, 1910

Ellipsoidella $\mathrm{sp}$.

(P1. 4, fig. 7)

Occurrence. Late Miocene-Middle Pleistocene; ODP holes 994C, 997A.

Remarks. Not enough specimens found to assign any species name.

Genus Ellipsoglandulina (Silvestri, 1900)

Ellipsoglandulina laevigata (Silvestri, 1900)

(P1. 4, fig. 8)

1900 Glandulina laevigata Silvestri: 1-9.

Occurrence. Late Miocene-Middle Pleistocene; ODP hole 994C.

Genus Ellipsopolymorphina Silvestri, 1901

Ellipsopolymorphina sp.

(P1. 4, fig. 9)

Occurrence. Middle Pliocene; ODP hole 994C.

Remarks. Not enough specimens found to assign any species name.

Genus Elphidium de Montfort, 1808

Elphidium incertum (Willamson, 1858)

(P1. 4, fig. 10)

1858 Polystomella umbilicatula var. incerta Willamson: 44, pl. 3, fig. $82 \mathrm{a}$.

Occurrence. Early Pliocene-Holocene; ODP holes 991A, 994C, 995A, 995B, 997A.

Genus Epistominella Husezima \& Maruhasi, 1944

Epistominella exigua (Brady, 1884)

(P1. 4, figs 11-12)

1884 Pulvinulina exigua Brady: 696, pl. 103, figs 13-14.

Occurrence. Late Miocene-Holocene; ODP holes 991A, 994C, 995A, 995B, 997A.

Genus Evolvocassidulina Eade, 1967

Evolvocassidulina bradyi (Cushman, 1923)

(P1. 4, fig. 13) 


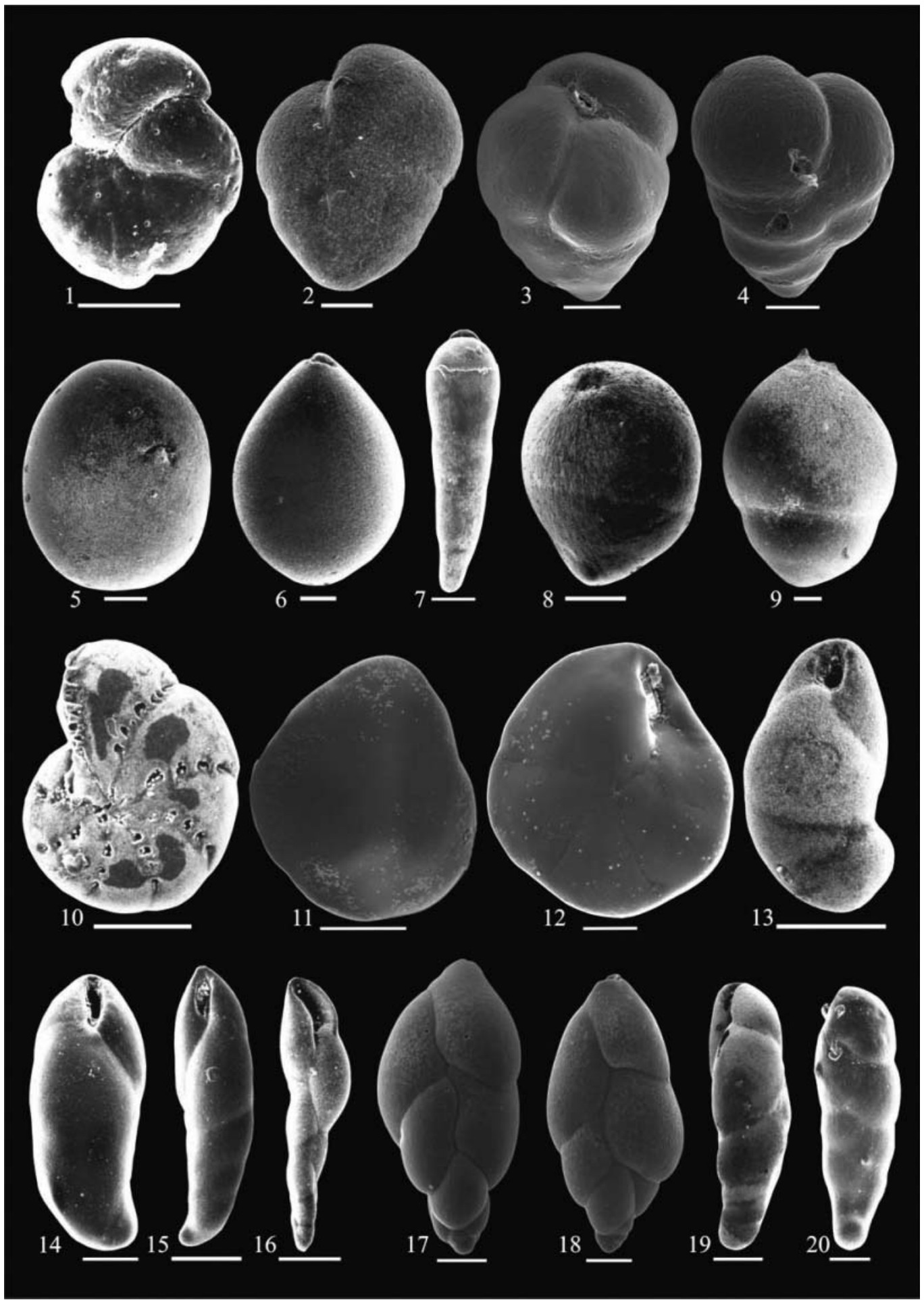

Explanation of Plate 4.

fig. 1. Discopulvinulina bertheloti, 164-994C, 25X-1, 75-77, umbilical view. fig. 2. Dorothia brevis, 164-994C, 34X-1, 4-6, side view. figs 3, 4. Eggerella bradyi, 164-995A: 3, 12H-5, 145-147, apertural view; 4, 5H-6, 148-150, side view. fig. 5. Ellipsobulimina seguenzai, 164-994C, 40X-2, 52-54, front view. fig. 6. Ellipsobulimina sp., 164-994C, 79X-1, 28-30, front view. fig. 7. Ellipsoidella sp., 164-994C, 40X-2, 52-54, front view. fig. 8. Ellipsoglandulina laevigata, 164-994C, 57X-2, 72-74, front view. fig. 9. Ellipsopolymorphina sp., 164-994C, 32X-1, 48-50, side view. fig. 10. Elphidium incertum, 164-994C, 22X-7, 70-72, side view. figs 11, 12. Epistominella exigua, 164-995A, 12H-5, 145-147: 11, spiral view; 12, umbilical view. fig. 13. Evolvocassidulina bradyi, 164-994C, 22X-1, 52-54, front view. figs 14, 15. Fursenkoina bradyi, 164-994C: 14, 21X-4, 75-77, front view; 15, 1H-1, 0-2, front view. fig. 16. Fursenkoina complanata, 164-994C, 17H-4, 74-76, side view. figs 17, 18. Fursenkoina fusiformis, 164-995A: 17, 5H-6, 148-150, side view; 18, 3H-2, 148-150, side view. figs 19, 20. Fursenkoina texturata, 164-994C: 19, 46X-3, 75-77, side view; 20, 21X-4, 75-77, side view. Scale bar $50 \mu \mathrm{m}$ for figs $12,17-18 ; 100 \mu \mathrm{m}$ for the rest. 
1923 Virgulina bradyi Cushman: 115, pl. 24, fig. 1.

Occurrence. Late Miocene-Holocene; ODP holes 991A, 994C, 995A, 995B, 997A.

Genus Fursenkoina Loeblich \& Tappan, 1961

Fursenkoina bradyi (Cushman, 1922)

$$
\text { (P1. 4, figs 14-15) }
$$

1922 Virgulina bradyi Cushman: 115, pl. 2, fig. 2.

Occurrence. Late Miocene-Holocene; ODP holes 991A, 994C, 995A, 995B.

\section{Fursenkoina complanata (Egger, 1893)}

(P1. 4, fig. 16)

1893 Virgulina complanata Egger: 292, pl. 8, figs 91-92.

Occurrence. Late Miocene-Late Pleistocene; ODP holes 994C, 997A.

Fursenkoina fusiformis (Williamson, 1858)

(Pl. 4, figs 17-18)

1858 Stainforthia fusiformis (Williamson); Bulimina pupoides d'Orbigny Var. fusiformis Williamson: 63, pl. 5, figs 129-130.

Occurrence. Late Miocene-Holocene; ODP holes 991A, 994C, 995A, 995B, 997A.

Fursenkoina texturata (Brady, 1884)

(Pl. 4, figs 19-20)

1884 Virgulina texturata Brady: 52, fig. 6.

Occurrence. Late Miocene-Holocene; ODP holes 991A, 994C, 995A, 995B, 997A.

Genus Gaudryina d’Orbigny, 1839

Gaudryina solida (Schwager, 1866)

(Pl. 5, fig. 1)

1866 Gaudryina solida Schwager: 199, pl. 4, fig. 11a-c.

Occurrence. Late Pliocene; ODP hole 994C.

Genus Gavelinopsis Hofker, 1951

Gavelinopsis lobatulus (Parr, 1950)

(P1. 5, figs 2-3)

1950 Discorbis lobatulus Parr: 354, pl. 13, figs 23-25.

Occurrence. Late Miocene-Holocene; ODP holes 991A, 994C, 995A, 995B, 997A.
Genus Glandulina d'Orbigny, 1839

Glandulina laevigata (d'Orbigny, 1826)

(Pl. 5, fig. 4)

1826 Nodosaria (Glandulina) laevigata d'Orbigny: 252, pl. 10, figs $1-4$.

Occurrence. Late Miocene-Late Pleistocene; ODP holes 991A, 994C, 995A, 995B, 997A.

Genus Globobulimina Cushman, 1927

Globobulimina notovata (Chapman, 1941)

(Pl. 5, figs 5-6)

1941 Globobulimina notovata Chapman: 166, pl. 50, fig. 13a-b.

Occurrence. Late Miocene-Holocene; ODP holes 991A, 994C, 995A, 995B.

Globobulimina pupoides (Cushman \& Parker, 1947)

$$
\text { (Pl. 5, fig. 7) }
$$

1947 Bulimina pupoides Cushman \& Parker: 83, pl. 19, figs 28-30; pl. 21, figs 1-2.

Occurrence. Early Pliocene-Middle Pleistocene; ODP holes 994C, 997A.

Globobulimina sp.

(P1. 5, figs 8-9)

Occurrence. Late Pliocene; ODP hole 994C.

Remarks. Not enough specimens found to assign any species name.

Genus Globocassidulina Voloshinova, 1960

Globocassidulina murrhina (Schwager, 1866)

(P1. 5, figs 10-11)

1866 Sphaeroidina murrhyna Schwager: 250, pl. 7, fig. 97.

Occurrence. Early Pliocene-Holocene, ODP holes 991A, 995A, 995B.

Globocassidulina obtusa (Williamson, 1858)

(Pl. 5, figs 12-13)

1858 Cassidulina obtusa Williamson: 69, pl. 6, figs 143-144.

Occurrence. Late Miocene-Holocene, ODP holes 991A, 994C, 995A, 995B, 997A.

Globocassidulina subglobosa ((Brady, 1884)

(P1. 5, figs 14-16) 


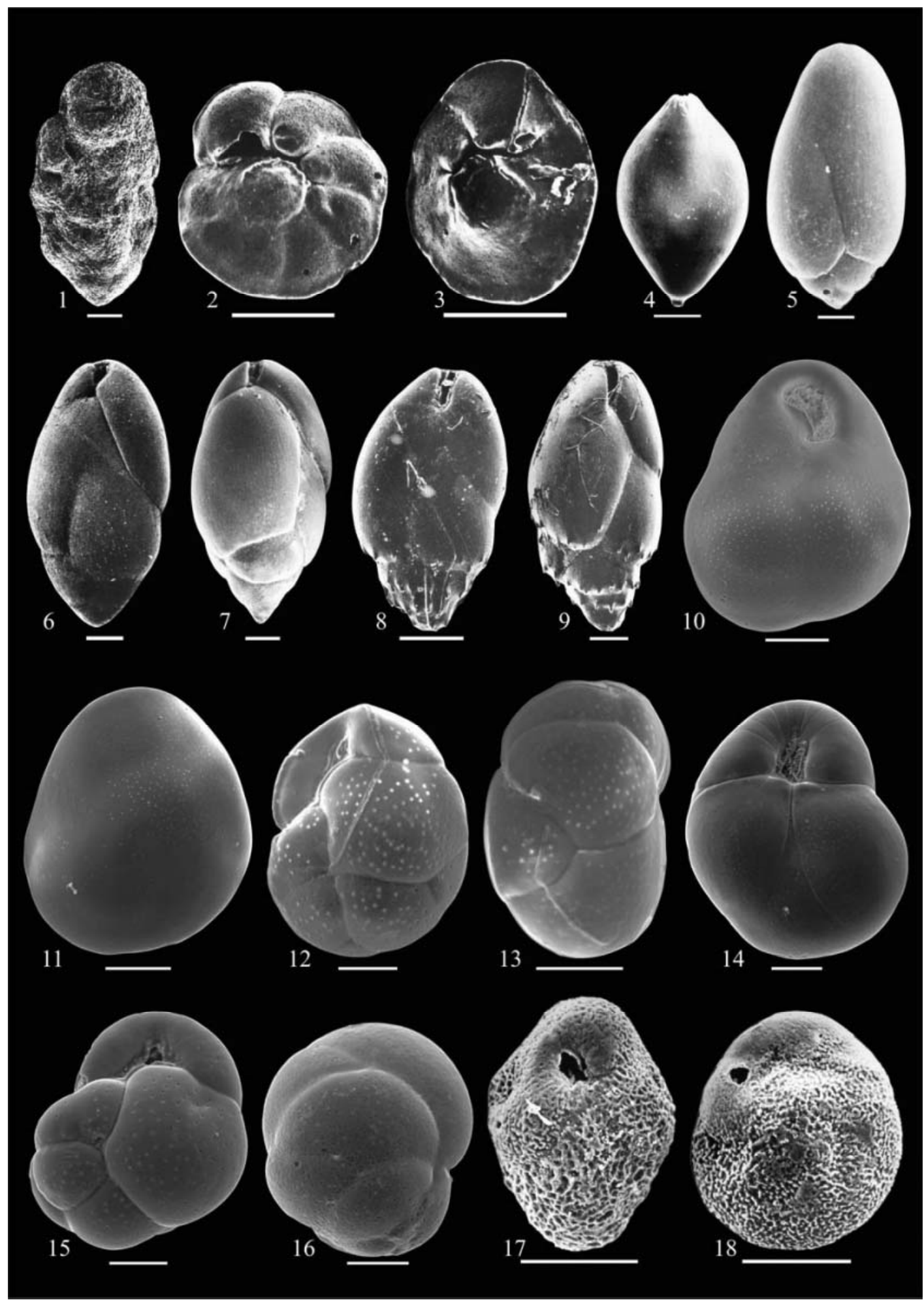

Explanation of Plate 5.

fig. 1. Gaudryina solida, 164-994C, 16H-CC, 15-17, side view. figs 2, 3. Gavelinopsis lobatulus, 164-994C, 16H-5, 75-77, umbilical view. fig. 4 Glandulina laevigata, 164-994C, 39X-1, 51-53, side view. figs 5, 6. Globobulimina notovata: 5, 164-995B, 2H-1, 75-77, side view; 6, 164-994C, 57X-2, 72-74, front view. fig. 7. Globobulimina pupoides, 164-994C, 17H-1, 74-76, front view. figs 8, 9. Globobulimina sp., 164-994C: 8, 37X-5, 10-12, front view; 9, 37X-1, 0-2, front view. figs 10, 11. Globocassidulina murrhina, 164-991A, 1H-1, 25-27: 10, front view; 11, back view. figs 12, 13. Globocassidulina obtusa, 164-991A, 4H-4, 147-149: 12, apertural view; 13, spiral view. figs 14-16. Globocassidulina subglobosa, 164-991A, 5H-4, 115-117: 14, 15, front view; 16, back view. figs 17, 18. Globocassidulina tumida, 164-994C: 17, 37X-1, 0-2, apertural view; 18, 42X-3, 70-72, side view. Scale bar $50 \mu \mathrm{m}$ for figs $12-13,15-16 ; 100 \mu \mathrm{m}$ for the rest. 
1884 Cassidulina subglobosa Brady: 430, pl. 54, figs 17a-c.

Occurrence. Late Miocene-Holocene, ODP holes 991A, 994C, 995A, 995B, 997A.

Globocassidulina tumida (Heron-Allen \& Earland, 1922)

$$
\text { (Pl. 5, figs 17-18) }
$$

1922 Cassidulina laevigata d'Orbigny Var. tumida Heron-Allen

\& Earland: 137, pl. 5, figs 8-10.

Occurrence. Late Miocene-Late Pleistocene; ODP holes 994C, 997A.

Genus Guttulina d'Orbigny, 1839

Guttulina sadoensis (Cushman \& Ozawa, 1930)

(P1. 6, fig. 1)

1928 Sigmomorpha sadoensis Cushman \& Ozawa: 17, pl. 2, fig. 11 .

Occurrence. Late Miocene; ODP hole 994C.

Genus Gyroidinoides Brotzen, 1942

Gyroidinoides broeckhianus (Karrer, 1878)

(Pl. 6, figs 2-4)

1878 Rotalia broeckhianus Karrer: 98, pl. 5, fig. 26.

Occurrence. Late Miocene-Late Pleistocene; ODP holes 991A, 994C, 995A, 997A.

Gyroidinoides cibaoensis (Bermúdez, 1949)

(Pl. 5, figs 5-6)

1949 Gyroidina cibaoensis Bermùdez: 252, pl. 17, figs 61-63.

Occurrence. Late Miocene-Holocene; ODP holes 991A, 994C, 995A, 995B, 997A.

\section{Gyroidinoides nitidula (Schwager, 1866)}

(Pl. 6, figs 7-8)

1866 Rotalia nitidula Schwager: 263, pl. 7, fig. 110.

Occurrence. Late Miocene-Holocene; ODP holes 991A, 994C, 995A, 995B, 997A.

Gyroidinoides polius (Phleger \& Parker, 1951)

(P1. 5, figs 9-10)

1951 Eponides polius Phelger \& Parker: 21, pl. 11, figs 1-2.

Occurrence. Late Miocene-Holocene; ODP holes 991A, 994C, 995A, 995B, 997A.
Genus Hagenowina Loeblich \& Tappan, 1961

Hagenowina sp.

(Pl. 6, figs 11-12)

Occurrence. Late Pliocene-Middle Pleistocene; ODP holes 994C, 997A.

Remarks. Not enough specimens found to assign any species name.

Genus Hoeglundina Brotzen, 1948

Hoeglundina elegans (d’Orbigny, 1826)

(P1. 6, figs 13-15)

1826 Rotalia (Turbulina) elegans d'Orbigny: 276, Mod. 54.

Occurrence. Late Miocene-Holocene; ODP holes 991A, 994C, 995A, 995B, 997A.

Genus Hormosina Brady, 1879

Hormosina sp.

(Pl. 7, fig. 1)

Occurrence. Late Miocene; ODP hole 994C.

Remarks. Not enough specimens found to assign any species name.

Genus Involvohauerina Loeblich \& Tappan, 1955

Involvohauerina $\mathrm{sp}$.

(Pl. 6, fig. 16)

Occurrence. Late Pliocene; ODP hole 994C.

Remarks. Not enough specimens found to assign any species name.

Genus Karreriella Cushman, 1933

Karreriella baccata (Schwager, 1866)

(Pl. 7, figs 2-3)

1866 Gaudryina baccata Schwager: 200, pl. 4, fig. 12a-b.

Occurrence. Middle Pleistocene-Late Pleistocene; ODP holes 994C, 997A.

Karreriella bradyi (Cushman, 1911)

(P1. 7, figs 4-5)

1911 Gaudryina bradyi Cushman: 67, pl. 2, fig. 107.

Occurrence. Late Miocene-Holocene; ODP holes 991A, 994C, 995A, 995B, 997A. 


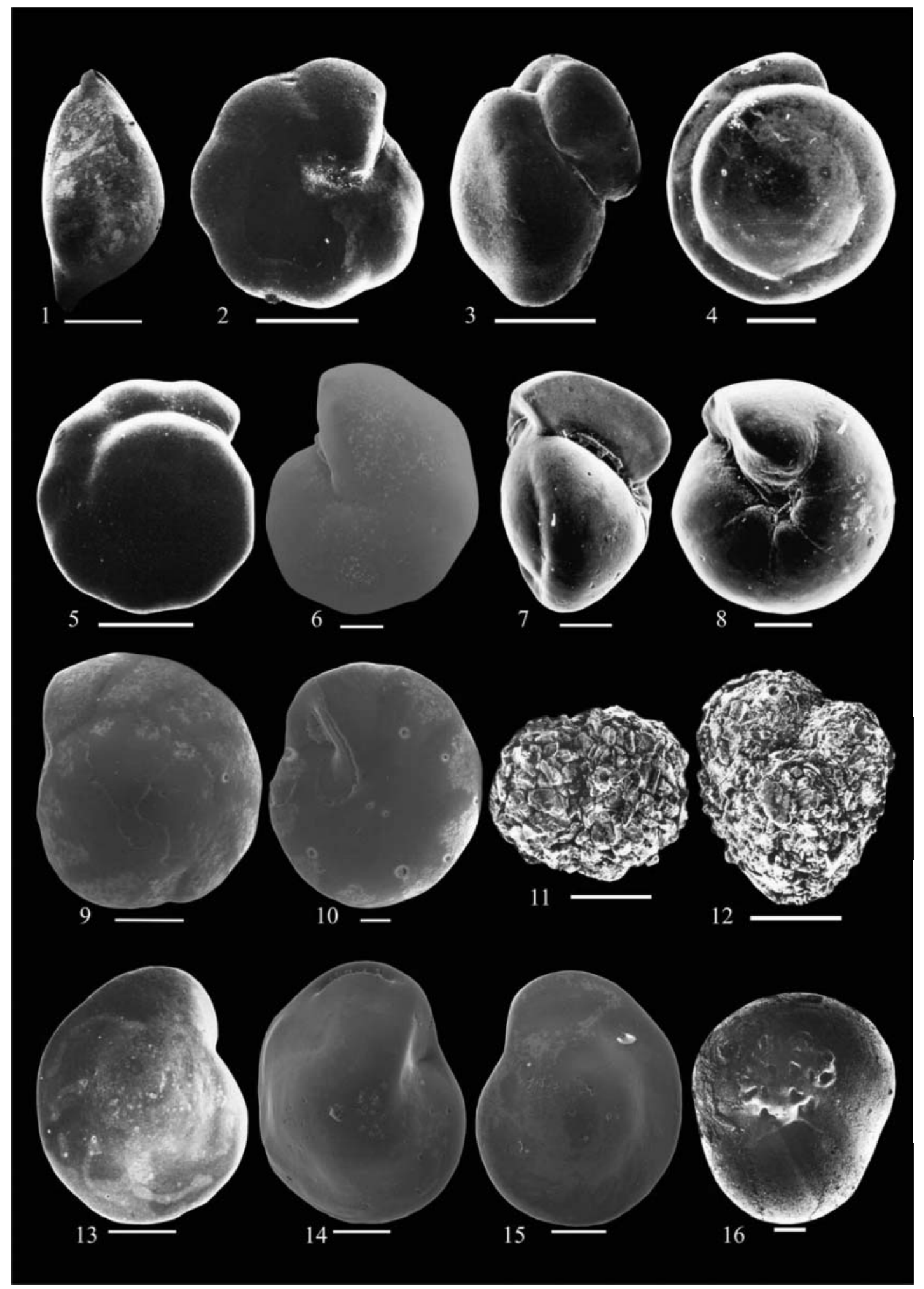

Explanation of Plate 6.

fig. 1. Guttulina sadoensis, 164-994C, 74X-5, 54-56, side view. figs 2-4. Gyroidinoides broeckhianus, 164-994C: 2, 4H-1, 75-77, umbilical view; 3, 4H-1, 75-77, side view; 4, 50X-2, 72-74, spiral view. figs 5, 6. Gyroidinoides cibaoensis: 5, 164-994C, 4H-1, 75-77, spiral view; 6, 164-991A, 1H-1, 25-27, umbilical view. figs 7, 8. Gyroidinoides nitidula; 164-994C: 7, 42X-5, 52-54, apertural view; 8, 35X-3, 52-54, umbilical view. figs 9, 10. Gyroidinoides polius, 164-995A, 2H-5, 75-77: 9, spiral view; 10, umbilical view. figs 11, 12. Hagenowina sp.,164-994C, 13H-5, 75-77: 11, apertural view; 12, side view. figs 13-15. Hoeglundina elegans: 13, 164-995B, 1H-CC, 18-20, spiral view; 14, 164-991A, 1H-1, 55-57, umbilical view, 15, 164-991A, 1H-1, 55-57, spiral view. fig. 16. Involvohauerina sp., 164-994C, 73X-1, 69-71, apertural view. Scale bar $20 \mu \mathrm{m}$ for fig. $10 ; 50 \mu \mathrm{m}$ for fig. 6; $100 \mu \mathrm{m}$ for the rest. 


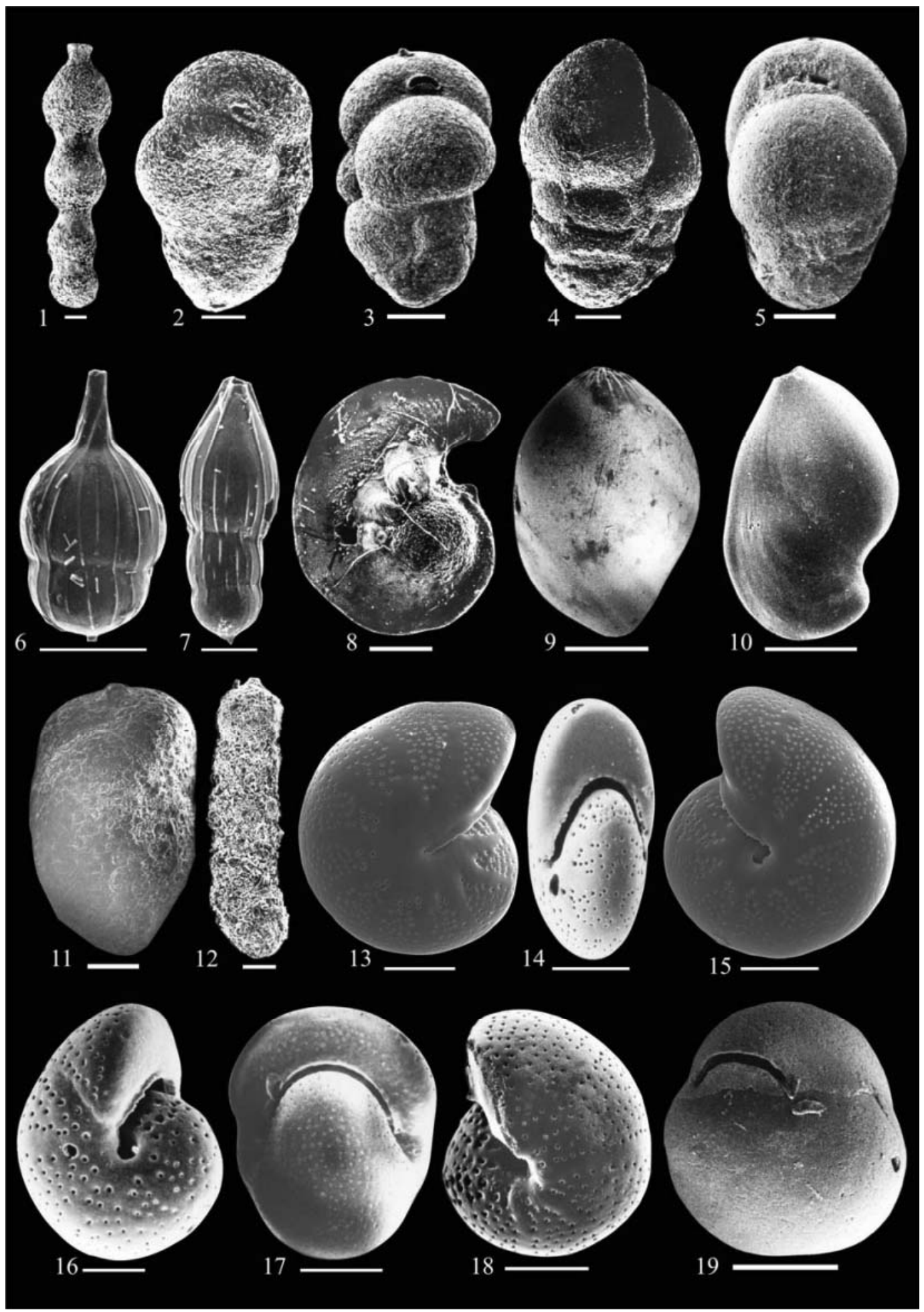

Explanation of Plate 7.

fig. 1. Hormosina sp., 164-994C, 68X-CC, 0-2, side view. figs 2, 3. Karreriella baccata, 164-994C, 10H-4, 75-77: 2, front view; 3, apertural view. figs 4, 5. Karreriella bradyi, 164-994C: 4, 67X-7, 30-32, side view; 5, 76X-6, 73-75, apertural view. figs 6, 7. Lagenodosaria sp., 164-997A: 6, 3H-2, 46-48, side view; 7, 2H-2, 46-48, side view. fig. 8. Laticarinina pauperata, 164-994C, 40X-2, 52-54, umbilical view. fig. 9. Marginulina obesa, 164-994C, 49X-5, 72-74, side view. fig. 10. Marginulinopsis bradyi, 164-994C, 50X-2, 72-74, side view. fig. 11. Martinottiella clarae, 164-997A, 4H-5, 45-47, side view. fig. 12. Martinottiella communis, 164-994C, 35X-CC, 0-2, side view. figs 13-15. Melonis barleeanum: 13, 164-995A, 4H-6, 148-150, side view; 14, 164-995B, 1H-CC, 18-20, apertural view; 15, 164-995A, 4H-6, 148-150, side view. figs 16-18. Melonis pompilioides: 16, 164-995B, 1H-5, 75-77, side view; 17, 164-995B, 1H-5, 75-77, apertural view; 18, 164-994C, 46X-2, 45-47, side view. fig. 19. Miliolinella subrotunda, 164-994C, 35X-2, 52-54, apertural view. Scale bar $50 \mu \mathrm{m}$ for fig. $16 ; 100 \mu \mathrm{m}$ for the rest. 
Deep-sea benthic foraminifera in the Neogene, NW Atlantic Ocean

Genus Lagenodosaria Silvestri, 1900

Lagenodosaria sp.

(Pl. 7, figs 6-7)

Occurrence. Early Pliocene, ODP hole 994C.

Remarks. Not enough specimens found to assign any species name.

Genus Laticarinina Galloway \& Wissler, 1927

Laticarinina pauperata (Parker \& Jones, 1865)

(Pl. 7, fig. 8)

1865 Pulvinulina repanda var. menardii sub var. pauperata Parker \& Jones: 395, pl. 6, figs 50-51.

Occurrence. Late Miocene-Late Pleistocene; ODP holes 991A, 994C, 995A, 995B, 997A.

Genus Marginulina d'Orbigny, 1826

Marginulina obesa (Cushman, 1923)

(Pl. 7, fig. 9)

1923 Marginulina obesa Cushman; 128, pl. 37, fig. 1.

Occurrence. Late Miocene-Late Pliocene; ODP holes 991A, 994C, 995A, 997A.

Genus Marginulinopsis Silvestri, 1904

Marginulinopsis bradyi (Goës, 1894)

(Pl. 7, fig. 10)

1894 Cristellaria bradyi Göes: 64, figs 11-12.

Occurrence. Late Miocene-Early Pleistocene; ODP holes 991A, 994C, 997A.

Genus Martinottiella Cushman, 1933

Martinottiella clarae (Gibson, 1967)

(P1. 7, fig. 11)

1967 Martinottiella clarae Gibson: 12, pl. 2, figs 24, 27-29.

Occurrence. Early Pleistocene-Middle Pleistocene; ODP holes 995A, 997A.

Martinottiella communis (d'Orbigny, 1846)

(Pl. 7, fig. 12)

1846 Clavulina communis d'Orbigny: 196, pl. 12, figs 1-2.

Occurrence. Late Miocene-Holocene; ODP holes 991A, 994C, 995B, 997A.
Genus Melonis de Montfort, 1808

Melonis barleeanum (Williamson, 1858)

(Pl. 7, figs 13-15)

1858 Nonionina barleeanum Williamson: 32, pl. 3, figs 68-69.

Occurrence. Late Miocene-Holocene; ODP holes 991A, 994C, 995A, 995B, 997A.

Melonis pompilioides (Fichtel \& Moll, 1798)

(P1. 7, figs 16-18)

1803 Nautilus pompilioides Fichtel \& Moll: 31, pl. 2, figs a-e.

Occurrence. Late Miocene-Holocene; ODP holes 991A, 994C, 995A, 995B, 997A.

Genus Miliolinella Wiesner, 1931

Miliolinella subrotunda (Montagu, 1803)

(Pl. 7, fig. 19)

1803 Vermiculum subrotunda Maontagu: pl. 1, fig. 11.

Occurrence. Late Miocene-Holocene; ODP holes 991A, 994C, 995A, 995B, 997A.

Genus Nodosaria Lamarck, 1812

Nodosaria acuminata (Hantken, 1875)

(Pl. 8, fig. 1)

1875 Nodosaria acuminata Hantken: 23, pl. 2, fig. 9.

Occurrence. Late Pliocene; ODP hole 994C.

Nodosaria brevicula (Schwager, 1866)

(P1. 8, figs 2-3)

1866 Nodosaria brevicula Schwager: 234, pl. 6, figs 71-72.

Occurrence. Early Pliocene-Late Pleistocene; ODP holes 991A, 994C, 995A, 997A.

Nodosaria holoserica (Schwager, 1866)

(P1. 8, fig. 4)

1866 Nodosaria holoserica Schwager: 221, pl. 5, fig. 49.

Occurrence. Early Pliocene-Early Pleistocene; ODP holes 994C, 997A.

\section{Nodosaria longiscata (d'Orbigny, 1846)}

(Pl. 8, fig. 5)

1846 Nodosaria longiscata d'Orbigny: 32, pl. 1, figs 10-12.

Occurrence. Late Miocene-Holocene; ODP holes 991A, 994C, 995B, 997A. 


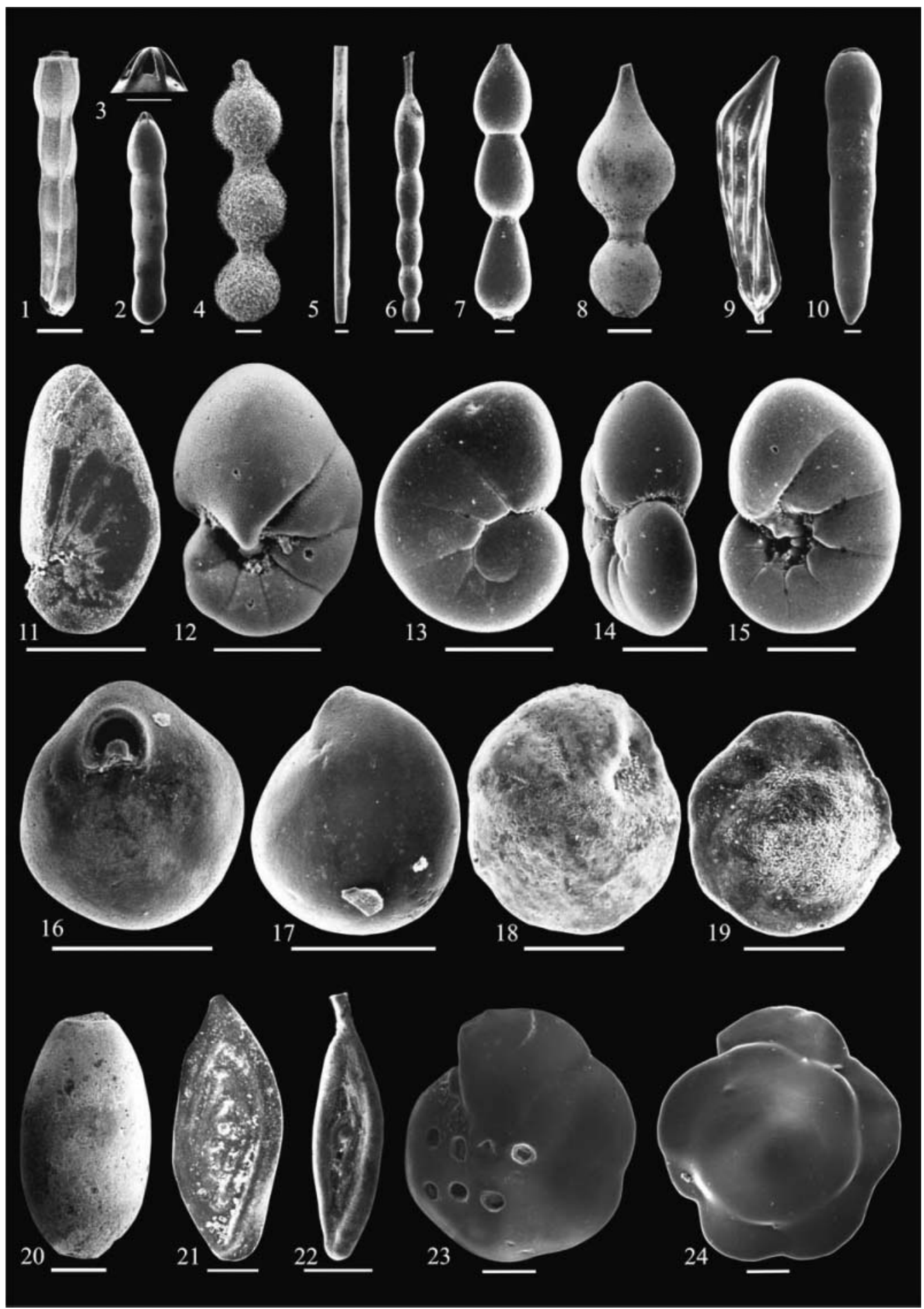

Explanation of Plate 8.

fig. 1. Nodosaria acuminata, 164-994C, 17H-2, 75-77, side view. figs 2, 3. Nodosaria brevicula, 164-994C, 13H-3, 74-76: 2, side view; 3, apertural view. fig. 4. Nodosaria holoserica, 164-994C, 35X-1, 51-53, side view. fig. 5. Nodosaria longiscata, 164-994C, 42X-3, 70-72, side view. fig. 6. Nodosaria ovicula, 164-994C, 16H-6, 74-76, side view. fig. 7. Nodosaria radicula, 164-994C, 17H-5, 74-76, side view. fig. 8. Nodosaria simplex, 164-994C, 63X-2, 72-74, side view. fig. 9. Nodosaria vertebralis, 164-994C, 23X-4, 70-72, side view. fig. 10. Nodosarella subnodosa, 164-997A, 34X-2, 10-12, side view. fig. 11. Nonion scaphum, 164-994C, 25X-1, 75-77, umbilical view. fig. 12. Nonionella auris, 164-994C, 47X-5, 52-54, umbilical view. figs 13-15. Nonionella bradyi, 164-994C, 1H-1, 0-2: 13, spiral view: 14, apertural view; 15, umbilical view. figs 16, 17. Nummoloculina irregularis, 164-994C, 25X-1, 75-77: 16, apertural view; 17, side view. figs 18, 19. Nuttallides umbonifera, 164-994C: 18, 22X-4, 70-72, umbilical view; 19, 24X-CC, 0-2, spiral view. fig. 20. Oolina botelliformis, 164-994C, 41X-7, 30-32, side view. fig. 21. Ophthalmidium acutimargo, 164-994C, 25X-4, 75-77, side view. fig. 22. Ophthalmidium pusillum, 164-994C, 31X-7, 52-54, side view. figs 23, 24. Oridorsalis umbonatus, 164-995A, 10H-5, 148-150: 23, umbilical view; 24, spiral view. Scale bar $50 \mu \mathrm{m}$ for figs $23-24 ; 100 \mu \mathrm{m}$ for the rest. 
Deep-sea benthic foraminifera in the Neogene, NW Atlantic Ocean

Nodosaria ovicula (d'Orbigny, 1826)

(P1. 8, fig. 6)

1866 Nodosaria ovicula (d'Orbigny); 252, Parker, Jones \& Brady: (1871) pl. 9, fig. 36h, k.

Occurrence. Middle Pliocene-Early Pleistocene; ODP holes 991A, 994C, 995A, 997A.

\section{Nodosaria radicula (Linné, 1931)}

(P1. 8, fig. 7)

1932 Nodosaria radicula (Linné); Macfadyen: 67, pl. 2, fig. 14.

Occurrence. Early Pliocene-Late Pleistocene; ODP holes 991A, 994C, 995A, 997A.

\section{Nodosaria simplex (Silvestri, 1872)}

(P1. 8, fig. 8)

1872 Nodosaria simplex (Silvestri); Barker: 131, pl. 62, figs 4-6.

Occurrence. Late Miocene-Late Pleistocene; ODP holes 991A, 994C, 995A, 997A.

Nodosaria vertebralis (Batsch, 1791)

(P1. 8, fig. 9)

1971 Nautilus (Orthoceras) vertebralis Batsch: 3, pl. 2, fig. 6a-b.

Occurrence. Late Pliocene; ODP hole 994C.

Genus Nodosarella Rzehak, 1895

Nodosarella subnodosa (Guppy, 1894)

(Pl. 8, fig. 10)

1894 Ellipsoidina subnodosa Guppy: 650, pl. 41, fig. 12.

Occurrence. Early Pliocene; ODP hole 997A.

Genus Nonion de Montfort, 1808

Nonion scaphum (Fichtel \& Moll, 1798)

(Pl. 8, fig. 11)

1798 Nautilus scapha Fichtel \& Moll: 105, pl. 105, figs d-f.

Occurrence. Middle Pliocene-Middle Pleistocene; ODP holes 991A, 995A.

$$
\begin{aligned}
& \text { Genus Nonionella Cushman, } 1926 \\
& \text { Nonionella auris (d'Orbigny, 1839) }
\end{aligned}
$$

(Pl. 8, fig. 12)

1839 Valvulina auris d'Orbigny: 47 pl. 5, figs 15-17.

Occurrence. Late Miocene-Holocene; ODP holes 991A, 994C, 995A, 995B, 997A.
Nonionella bradyi (Chapman, 1916)

(P1. 8, figs 13-15)

1916 Nonionella scapha var. bradii Chapman: 71, pl. 5, fig. 42.

Occurrence. Early Pleistocene-Holocene; ODP holes 991A, 994C, 995A.

Genus Nummoloculina Steinmann, 1881

Nummoloculina irregularis (d'Orbigny, 1839)

(Pl. 8, figs 16-17)

1839 Biloculina irregularis d'Orbigny: 67, pl. 8, figs 20-21.

Occurrence. Late Miocene-Holocene; ODP holes 991A, 994C, 995A, 997A.

Genus Nuttallides Finlay 1939

Nuttallides umbonifera (Cushman, 1933)

(P1. 8, figs 18-19)

1933 Pulvinulinella umbonifera Cushman: 90, pl. 9, fig. 9.

Occurrence. Late Miocene-Holocene; ODP holes 991A, 994C, 995A, 995B, 997A.

Genus Oolina d'Orbigny, 1839

Oolina botelliformis ((Brady, 1884)

(Pl. 8, fig. 20)

1884 Oolina botelliformis (Brady); Barker: 115, pl. 55, fig. 6.

Occurrence. Late Miocene-Early Pliocene; ODP hole 994C.

Genus Ophthalmidium Kübler \& Zwingli, 1870

Ophthalmidium acutimargo (Brady, 1884)

(P1. 8, fig. 21)

1884 Spiroloculina acutimargo Brady: 154, pl. 10, figs 12-15.

Occurrence. Late Miocene-Late Pleistocene; ODP holes 994C, 995A, 997A.

Ophthalmidium pusillum (Earland, 1943)

(P1. 8, fig. 22)

1934 Spiroloculina pusilla Earland: 10, 47.

Occurrence. Late Miocene-Late Pleistocene; ODP holes 994C, 995A, 997A.

Genus Oridorsalis Andersen, 1961

Oridorsalis umbonatus (Reuss, 1851)

(P1. 8, figs 23-24) 
1851 Rotalina umbonata Reuss: 75, pl. 5, fig. 35 a-c.

Occurrence. Late Miocene-Holocene; ODP holes 991A, 994C, 995A, 995B, 997A.

Genus Orthomorphina Stainforth, 1952

Orthomorphina challengeriana (Thalmann, 1937)

(Pl. 9, fig. 1)

1937 Nodogenerina challengeriana Thalmann: 341, pl. 64, figs 25-27.

Occurrence. Late Miocene-Late Pleistocene; ODP holes 991A, 994C, 995A, 995B, 997A.

Orthomorphina glandigena (Schwager, 1866)

(P1. 9, fig. 2)

1866 Nodosaria glandigena Schwager: 219, pl. 4, fig. 46.

Occurrence. Early Pliocene-Early Pleistocene; ODP holes 994C, 997A.

Orthomorphina koina (Schwager, 1866)

(Pl. 9, fig. 3)

1866 Nodosaria koina Schwager: 220, pl. 5, fig. 47.

Occurrence. Early Pliocene-Middle Pleistocene; ODP holes 991A, 995A, 997A.

Orthomorphina modesta (Bermudez, 1937)

(Pl. 9, fig. 4)

1937 Ellipsonodosaria modesta Bermudez: 238, pl. 20, fig. 3.

Occurrence. Late Miocene-Late Pleistocene; ODP holes 991A, 994C, 995A.

Genus Parafrondicularia Asano, 1938

Parafrondicularia advena (Cushman, 1923)

(Pl. 9, fig. 5)

1938 Frondicularia advena Cushman: 188, pl. 24, fig. 17.

Occurrence. Late Miocene-Late Pleistocene; ODP holes 991A, 994C, 995A, 995B, 997A

Genus Planulina d'Orbigny, 1826

Planulina ariminensis (d'Orbigny, 1826)

(P1. 9, fig. 6)

1826 Planulina ariminensis d'Orbigny: 280, pl. 14, fig. 13.

Occurrence. Early Pliocene-Late Pleistocene; ODP holes 991A, 995A, 995B, 997A.
Genus Pleurostomella Reuss, 1860

Pleurostomella acuminata (Cushman, 1922) (Pl. 9, figs 7-8)

1922 Pleurostomella acuminata Cushman: 50, pl. 19, fig. 6.

Occurrence. Late Miocene-Late Pleistocene; ODP holes 991A, 994C.

Pleurostomella acuta (Hantken, 1875)

(Pl. 9, fig. 9)

1875 Pleurostomella acuta Hantken: 44, pl. 13, fig. 18.

Occurrence. Late Miocene-Holocene; ODP holes 991A, 994C, 995A, 995B, 997A.

Pleurostomella alternans (Schwager, 1866)

(Pl. 9, fig. 10)

1866 Pleurostomella alternans Schwager: 238, pl. 6, figs 79-80.

Occurrence. Late Miocene-Holocene; ODP holes 991A, 994C, 995A, 995B, 997A.

Pleurostomella brevis (Schwager, 1866)

(Pl. 9, figs 11-12)

1866 Pleurostomella brevis Schwager: 236, pl. 6, fig. 81.

Occurrence. Late Miocene-Early Pleistocene; ODP holes 991A, 994C, 995A, 995B, 997A.

Pleurostomella rapa (Gümbel, 1868)

(P1. 9, fig. 13)

1868 Pleurostomella rapa Gümbel; var. recens Dervieux (1899): $76-78$.

Occurrence. Late Miocene-Late Pliocene; ODP holes 994C, 995A.

Pleurostomella sapperi (Schubert, 1911)

(P1. 9, figs 14-15)

1911 Pleurostomella sapperi Schubert: 56, fig. 3a-b.

Occurrence. Middle Pliocene-Early Pleistocene; ODP holes 994C, 995A, 997A.

Pleurostomella subnodosa (Reuss, 1851)

(P1. 9, fig. 16)

1851 Pleurostomella subnodosa Reuss: 24, pls 2-6.

Occurrence. Late Miocene-Late Pleistocene; ODP holes 994C, 997A. 


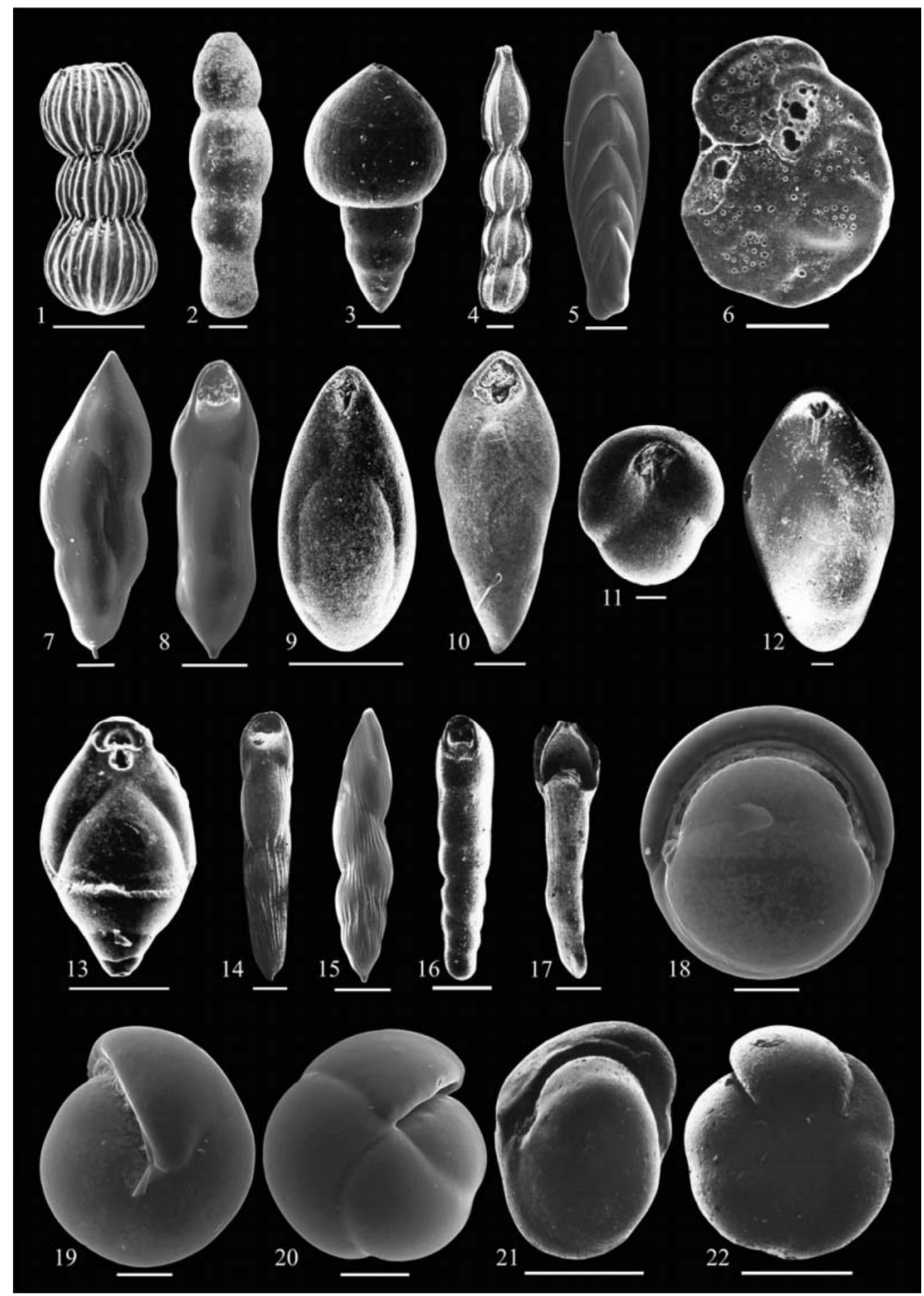

Explanation of Plate 9.

fig. 1. Orthomorphina challengeriana, 164-994C, 39X-CC, 0-2, side view. fig. 2. Orthomorphina glandigena, 164-994C, 51X-2, 72-74, side view. fig. 3. Orthomorphina koina, 164-997A, 5H-2, 45-47, side view. fig. 4. Orthomorphina modesta, 164-994C, 41X-4, 10-12, side view. fig. 5. Parafrondicularia advena, 164-995A, 4H-6, 148-150, front view. fig. 6. Planulina ariminensis, 164-997A, 11H-7, 45-47, spiral view. figs 7, 8. Pleurostomella acuminata, 164-995A: 7, 15H-7, 75-77, side view; 8, 10H-7, 75-77, front view. fig. 9. Pleurostomella acuta, 164-994C, 22X-4, 70-72, front view. fig. 10. Pleurostomella alternans, 164-994C, 40X-3, 52-54, front view. figs 11, 12. Pleurostomella brevis, 164-994C: 11, 84X-CC, 2-4, apertural view; 12, 22X-1, 52-54, front view. fig. 13. Pleurostomella rapa, 164-994C, 38X-CC, 0-2, front view. figs 14, 15. Pleurostomella sapperi: 14, 164-994C, 16H-2, 75-77, front view; 15, 164-995A, 7H-5, 75-77, side view. fig. 16. Pleurostomella subnodosa, 164-994C, 76X-7, 4-6, front view. fig. 17. Pseudonodosaria sp., 164-994C, 74X-3, 71-73, apertural view. figs 18, 19. Pullenia bulloides, 164-995A, 2H-5, 75-77: 18, apertural view; 19, side view. figs 20-22. Pullenia osloensis: 20, 164-995A, 3H-2, 148-150, side view; 21, 164-994C, 81X-1, 4-6, apertural view; 22, 164-994C, 81X-5, 72-74, side view. Scale bar $50 \mu \mathrm{m}$ for figs $7,18-20 ; 200 \mu \mathrm{m}$ for fig. $15 ; 100 \mu \mathrm{m}$ for the rest. 
Genus Pseudonodosaria Boomgaart 1949

Pseudonodosaria sp.

(Pl. 9, fig. 17)

Occurrence. Late Miocene- Early Pleistocene; ODP hole 994C.

Remarks. Not enough specimens found to assign any species name.

Genus Pullenia Parker \& Jones, 1862

Pullenia bulloides (d'Orbigny, 1846)

$$
\text { (P1. 9, figs 18-19) }
$$

1846 Nonionina bulloides d'Orbigny: 107, pl. 5, figs 9-10.

Occurrence. Late Miocene-Holocene; ODP holes 991A, 994C, 995A, 995B, 997A.

Pullenia osloensis (Feyling-Hanssen, 1954)

(P1. 9, figs 20-21)

1954 Pullenia quinqueloba Reuss; subp. minuta Feyling-Hanssen: 194, figs 3-4.

Occurrence. Late Miocene-Holocene; ODP holes 991A, 994C, 995A, 995B, 997A.

\section{Pullenia quinqueloba (Reuss, 1851)}

(P1. 10, figs 1-2)

1851 Nonionina quinqueloba Reuss: 71, pl. 5, fig. 31.

Occurrence. Late Miocene-Holocene; ODP holes 991A, 994C, 995A, 995B, 997A.

Pullenia salisburyi (Stewart \& Stewart, 1930)

(Pl. 10, figs 3-4)

1930 Pullenia salisburyi Stewart \& Stewart: 72, pl. 8, fig. 2a-b.

Occurrence. Late Miocene-Holocene; ODP holes 991A, 994C, 995A, 995B.

Genus Pyrgo Defrance, 1824

Pyrgo depressa (d'Orbigny, 1826)

(Pl. 10, fig. 5)

1826 Biloculina depressa d'Orbigny: 298, mod no. 91.

Occurrence. Late Miocene-Holocene; ODP holes 991A, 994C, 995A, 995B, 997A.

\section{Pyrgo lucernula (Schwager, 1866)}

(P1. 10, figs 6-8)

1866 Biloculina lucernula Schwager: 202, pl. 4, fig. 17a-b.
Occurrence. Late Miocene-Holocene; ODP holes 991A, 994C, 995A, 995B, 997A.

Pyrgo murrhina (Schwager, 1866)

(P1. 10, figs 9-10)

1866 Biloculina murrhina Schwager: 203, pl. 4, fig. 15.

Occurrence. Late Miocene-Holocene; ODP holes 991A, 994C, 995A, 995B, 997A.

Pyrgo serrata (L. W. Bailey, 1861)

(P1. 10, figs 11-12)

1861 Biloculina serrata Bailey: 350, pl. 8, fig. E.

Occurrence. Late Miocene-Middle Pleistocene; ODP holes 994C, 995A, 997A.

Pyrgo vespertilio (Schlumberger, 1891)

(Pl. 10, fig. 13)

1891 Biloculina vespertilio Schlumberger: 174, pl. 10, figs 74-76.

Occurrence. Late Miocene-Middle Pleistocene; ODP hole 995A.

Genus Pyrulina d'Orbigny, 1839

Pyrulina cylindroides (Roemer, 1838)

(Pl. 10, fig. 14)

1838 Polymorphina cylindroides Roemer: 386, pl. 3, fig. 26a-b.

Occurrence. Late Miocene-Late Pliocene; ODP holes 994C, 995B, 997A.

Pyrulina extensa (Cushman, 1923)

(P1. 10, fig. 15)

1923 Polymorphina extensa Cushman: 156, pl. 4, figs 7-8.

Occurrence. Early Pliocene-Early Pleistocene; ODP hole 994C.

Pyrulina fusiformis (Roemer, 1938)

(Pl. 10, fig. 16)

1838 Polymorphina fusiformis Roemer: 386, pl. 3, fig. 37.

Occurrence. Late Miocene-Middle Pleistocene; ODP holes 991A, 994C, 995A, 997A.

Genus Quadrimorphina Finlay, 1939

Quadrimorphina laevigata (Phleger \& Parker, 1951)

(Pl. 11, figs 1-2)

1951 Valvulineria laevigata Phelger \& Parker: 25, pl. 13, figs $11-12$. 


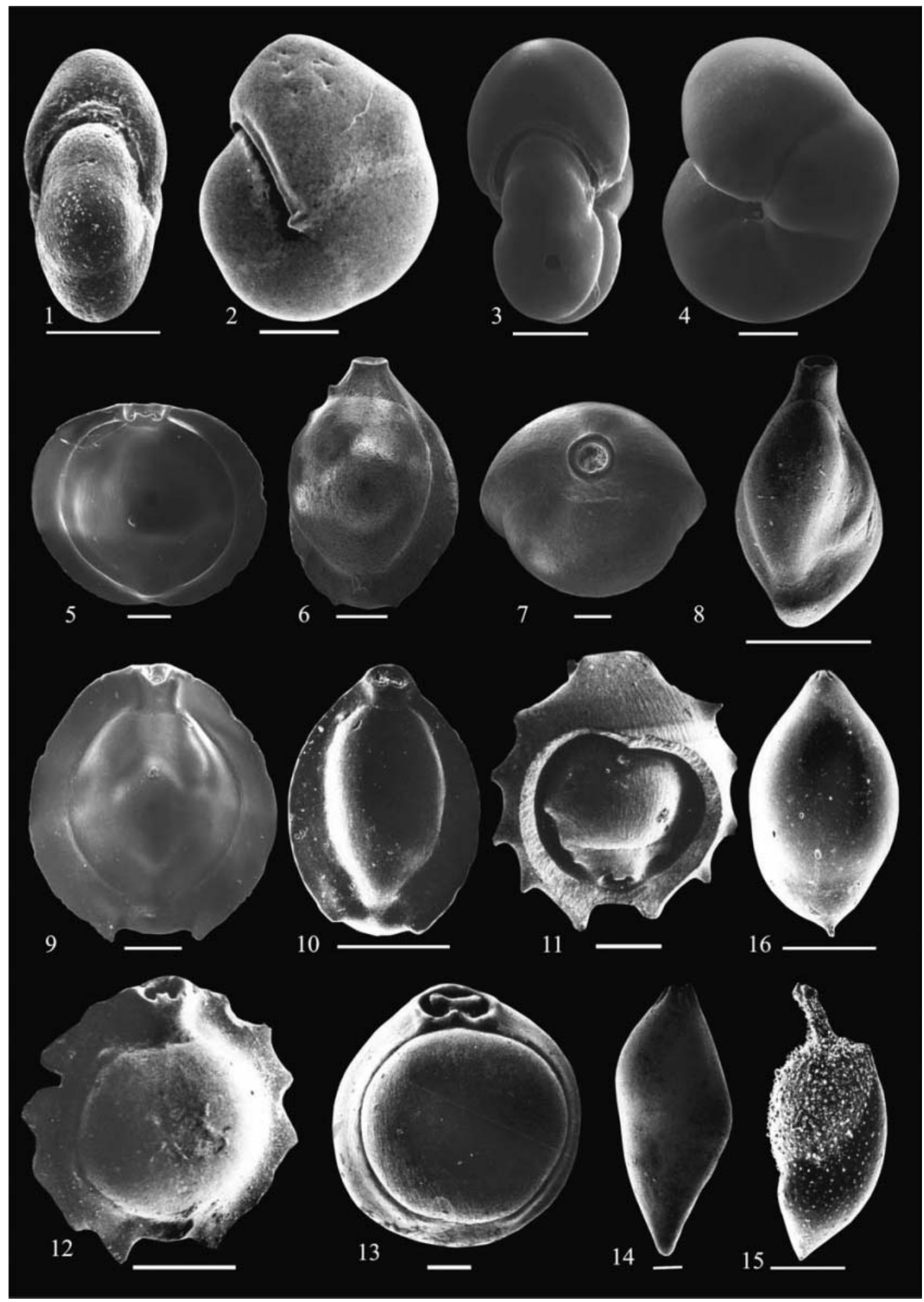

Explanation of Plate 10.

figs 1, 2. Pullenia quinqueloba, 164-994C, 35X-1, 0-2: 1, apertural view; 2, side view. figs 3, 4. Pullenia salisburyi, 164-995A, 4H-6, 148-150; 3, apertural view; 4, side view. fig. 5. Pyrgo depressa, 164-991A, 1H-1, 25-27, apertural view. figs 6-8. Pyrgo lucernula: 6, 164-995A, 15H-7, 71-77, side view; 7, 164-995A, 15H-7, 71-77, apertural view; 8, 164-994C, 25X-4, 75-77, front view. figs 9, 10. Pyrgo murrhina: 9, 164-995A, 10H-5, 148-150, front view; 10, 164-994C, 35X-2, 52-54, front view. figs 11, 12. Pyrgo serrate, 164-994C: 11, 58X-CC, 0-2, back view; 12, 64X-5, 72-74, front view. fig. 13. Pyrgo vespertilio, 164-994C, 17H-7, 52-54, front view. fig. 14. Pyrulina cylindroides, 164-994C, 61X-1, 72-74, side view. fig. 15. Pyrulina extensa, 164-994C, 20X-5, 75-77, side view. fig. 16. Pyrulina fusiformis, 164-994C, 17H-CC, 0-2, side view. Scale bar $50 \mu \mathrm{m}$ for fig. 4; $200 \mu \mathrm{m}$ for fig. $7 ; 100 \mu \mathrm{m}$ for the rest. 

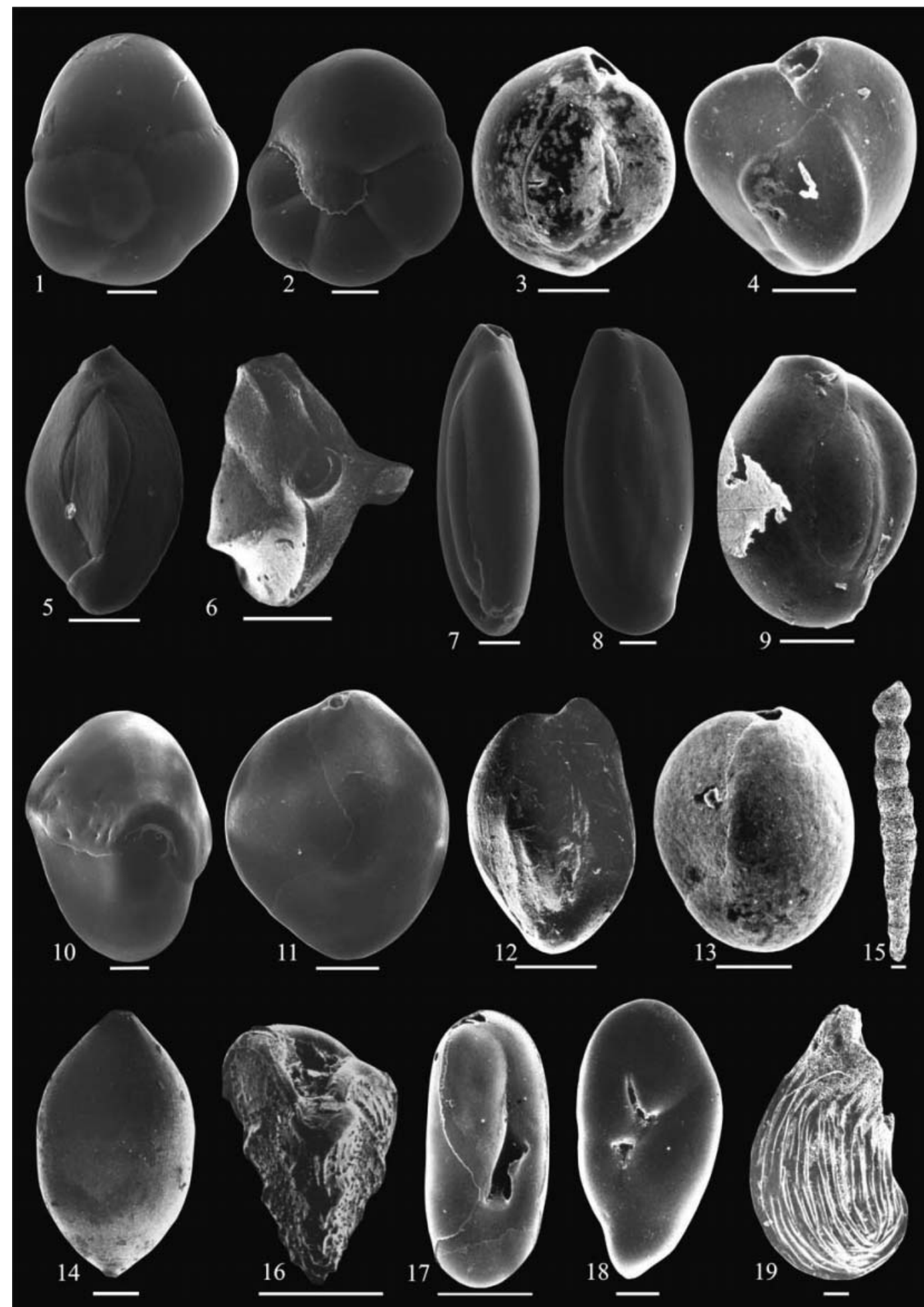

Explanation of Plate 11

figs 1, 2. Quadrimorphina laevigata, 164-995A, 3H-2, 148-150: 1, spiral view; 2, umbilical view. figs 3, 4. Quinqueloculina auberiana, 164-994C, 35X-2, 52-54, side view. figs 5, 6. Ouinqueloculina lamarckiana: 5, 164-995A, 8H-4, 75-77, side view; 6, 164-994C, 74X-3, 71-73, apertural view. figs 7, 8. Quinqueloculina oblonga, 164-995A, 3H-2, 148-150, side view. figs 9-11. Quinqueloculina seminulum: 9, 164-994C, 34X-1, 4-6, side view, 10, 164-995A, 15H-7, 75-77, side view; 11, 164-995A, 15H-7, 75-77, apertural view. fig. 12. Quinqueloculina stalkeri, 164-994C, 62X-1, 72-74, side view. fig. 13. Quinqueloculina weaver, 164-994C, 40X-1, 54-56, side view. fig. 14. Rectoglandulina armata, 164-994C, 72X-CC, 0-2, side view. fig. 15. Reophax bacillaris, 164-997A, 5H-4, 45-47, side view. fig. 16. Reussella simplex, 164-994C, 12H-5, 75-77, front view. fig. 17. Robertina subcylindrica, 164-994C, 1H-1, 0-2, apertural view; fig. 18. Robertinoides bradyi, 164-994C, 1H-1, 0-2, apertural view. fig. 19. Robulus costatus, 164-997A, 10H-3, 45-47, side view. Scale bar $50 \mu \mathrm{m}$ for figs $1-2,7-8,11 ; 100 \mu \mathrm{m}$ for the rest. 
Deep-sea benthic foraminifera in the Neogene, NW Atlantic Ocean

Occurrence. Late Miocene-Holocene; ODP holes 991A, 994C, Occurrence. Late Miocene; ODP hole 994C. 995A, 995B, 997A.

Genus Quinqueloculina d'Orbigny, 1826

Quinqueloculina auberiana (d'Orbigny, 1839)

(Pl. 11, figs 3-4)

1839 Miliolona auberiana d'Orbigny; 167, pl. 7, figs 1-3.

Occurrence. Middle Pliocene; ODP hole 994C.

Quinqueloculina lamarckiana (d'Orbigny, 1839)

(P1. 11, figs 5-6)

1839 Quinqueloculina lamarckiana d'Orbigny: 189, pl. 11, figs 14-15.

Occurrence. Late Miocene-Holocene; ODP holes 991A, 994C, 995A, 995B, 997A.

Quinqueloculina oblonga (Montagu, 1803)

(Pl. 11, figs 7-8)

1803 Vermiculum oblongum Montagu: 522, pl. 14, fig. 9.

Occurrence. Late Pleistocene; ODP hole 991A.

Quinqueloculina seminulum (Linnaeus, 1758)

(P1. 11, figs 9-11)

1758 Serpula seminula Linné: 786, pl. 2, fig. 1a-c.

Occurrence. Late Miocene-Holocene; ODP holes 991A, 995A, 995B, 997A.

Quinqueloculina stalkeri (Loeblich \& Tappan, 1953)

(P1. 11, fig. 12)

1953 Quinqueloculina stalkeri Loeblich \& Tappan: 40, pl. 5, figs 5-9.

Occurrence. Early Pliocene-Late Pleistocene; ODP holes 994C, 995A, 997A.

Quinqueloculina weaveri (Rau, 1948)

(P1. 11, fig. 13)

1948 Quinqueloculina weaveri Rau: 159, pl. 28, figs 1-3.

Occurrence. Late Miocene-Holocene; ODP holes 991A, 994C, 995A, 995B, 997A.

Genus Rectoglandulina Loeblich \& Tappan, 1955

Rectoglandulina armata (Reuss, 1884)

(P1. 11, fig. 14)

1884 Nodosaria (Glandulina) armata (Brady); Barker: 133, pl. 63, fig. 6 .
Genus: Reophax de Montfort, 1808

Reophax bacillaris (Brady, 1884)

(Pl. 11, fig. 15)

1884 Reophax bacillaris Brady: 103, pl. 30, figs 23-24.

Occurrence. Middle Pleistocene; ODP hole 997A.

Genus Reussella Galloway, 1933

Reussella simplex (Cushman, 1929)

(Pl. 11, fig. 16)

1929 Trimosina simplex Cushman: 158, fig. 2a-b.

Occurrence. Early Pliocene-Holocene; ODP holes 991A, 994C, 995A, 997A.

Genus Robertina d'Orbigny, 1846

Robertina subcylindrica (Brady, 1881)

(Pl. 11, fig. 17)

1881 Bulimina subcylindrica (Brady); Barker: 103, pl. 50, fig.16.

Occurrence. Early Pleistocene-Holocene; ODP holes 991A, 994C, 995A, 995B.

Genus Robertinoides Höglund, 1947

Robertinoides bradyi (Cushman \& Parker, 1936)

(Pl. 11, fig. 18)

1936 Robertina bradyi Cushman \& Parker: p. 99, pl. 16, fig. $9 \mathrm{a}-\mathrm{b}$.

Occurrence. Late Miocene-Holocene; ODP holes 991A, 994C, 995A, 995B, 997A.

Genus Robulus de Montfort, 1808

Robulus costatus (Fichtel \& Moll)

(Pl. 11, fig. 19)

1944 Robulus costatus (Fichtel \& Moll); LeRoy: 77, pl. 3, figs $15-16$.

Occurrence. Early Pleistocene; ODP hole 997A.

Robulus gibbus (d'Orbigny, 1839)

(Pl. 12, fig. 1)

1839 Cristellaria gibba d'Orbigny: 63, pl. 7, figs 20-21.

Occurrence. Late Miocene-Holocene; ODP holes 991A, 994C, 995A, 995B, 997A. 


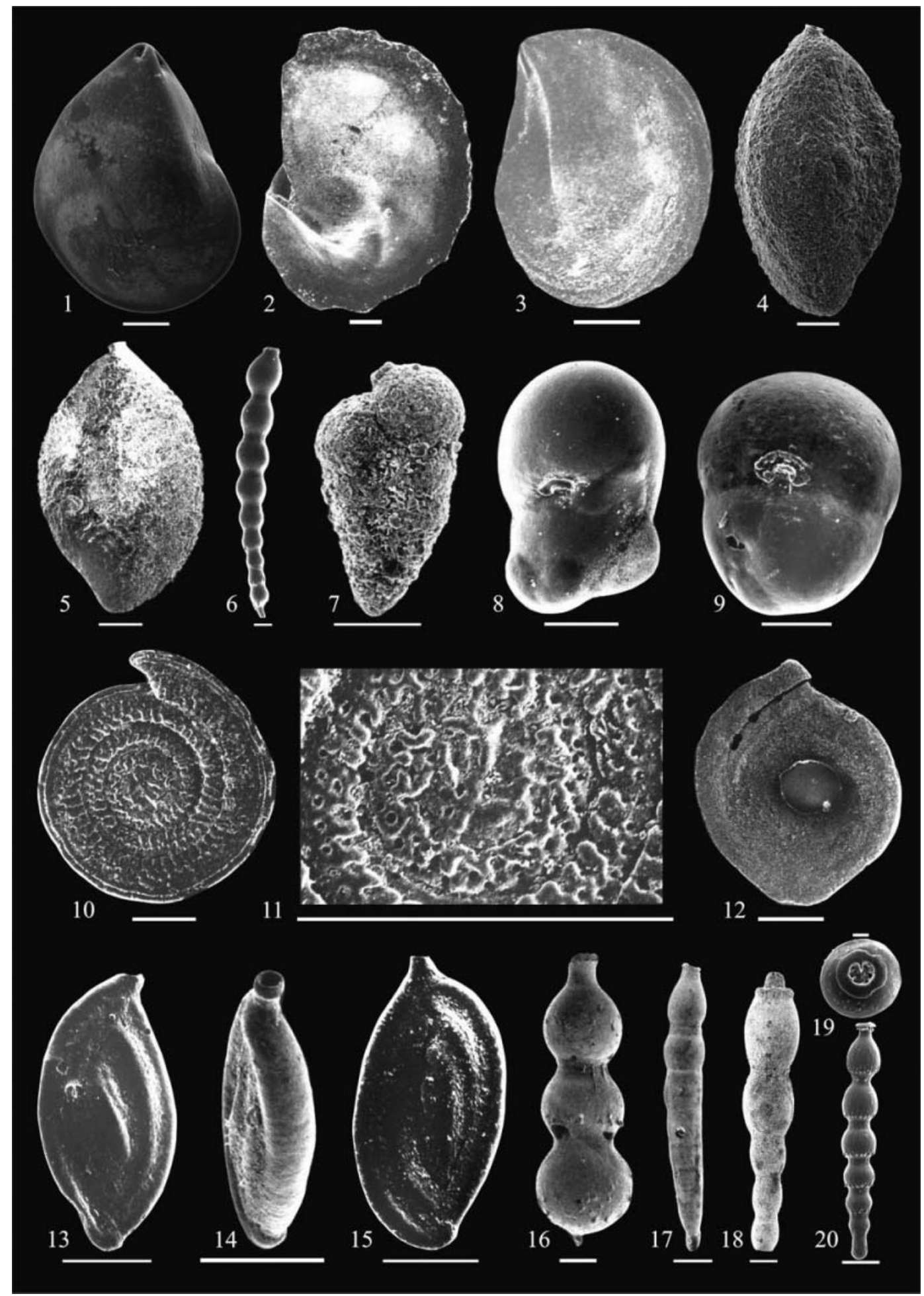

Explanation of Plate 12.

fig. 1. Robulus gibbus, 164-991A, 1H-2, 25-27, side view. fig. 2. Robulus iota, 164-997A, 22X-4, 10-12, side view; fig. 3. Robulus thalmanni, 164-994C, 58X-3, 47-49, side view. figs 4, 5. Sigmoilopsis schlumbergeri: 4, 164-995A, 2H-6, 75-77, side view; 5, 164-994C, 50X-3, 72-74, side view. fig. 6. Siphonodosaria pomuligera, 164-997A, 7H-1, 45-47, side view. fig. 7. Siphotextularia catenata, 164-994C, 43X-1, 52-54, side view. figs 8, 9. Sphaeroidina bulloides, 164-994C: 8, 26X-3, 51-53, apertural view; 9, 35X-3, 52-54, apertural view. figs 10, 11. Spirillina decorata, 164-994C, 83X-2, 76-78: 10, spiral view; 11, surface structure. fig. 12. Spiroloculina esnaensis, 164-994C, 63X-6, 71-73, spiral view. figs 13-15. Spiroloculina tenuis; 164-994C, 13H-1, 74-76: 13, 15, spiral view; 14, side view. fig. 16. Stilostomella abyssorum, 164-994C, 77X-4, 71-73, side view. fig. 17. Stilostomella consobrina, 164-994C, 38X-2, 0-2, side view. fig. 18. Stilostomella fistuca, 164-994C, 42X-2, 68-70, side view. figs 19, 20. Stilostomella lepidula, 164-991A, 2H-6, 55-57: 19, apertural view; 20, side view. Scale bar $20 \mu \mathrm{m}$ for fig. 19; $100 \mu \mathrm{m}$ for the rest. 
Deep-sea benthic foraminifera in the Neogene, NW Atlantic Ocean

Robulus iota (Cushman, 1923)

(P1. 12, fig. 2)

1923 Cristellaria iota Cushman: 111, pl. 29, fig. 2.

Occurrence. Early Pliocene; ODP hole 997A.

Robulus thalmanni (Hessland, 1943)

(P1. 12, fig. 3)

1943 Cristellaria rotulata Hessland: 265, pl. 2, fig. 16a-b.

Occurrence. Late Miocene-Late Pleistocene; ODP holes 994C, 997A.

Genus Sigmoilopsis Finlay, 1947

Sigmoilopsis schlumbergeri (Silvestri, 1904)

(P1. 12, figs 4-5)

1904 Sigmoilina schlumbergeri Silvestri: 267, 269, figs 6-9.

Occurrence. Late Miocene-Holocene; ODP holes 991A, 994C, 995A, 995B, 997A.

Genus Siphonodosaria Silvestri, 1924

Siphonodosaria pomuligera (Stache, 1864)

(P1. 12, fig. 6)

1864 Dentalina pomuligera Stache: 204, pl. 22, fig. 31.

Occurrence. Early Pleistocene; ODP hole 997A.

Genus Siphotextularia Finlay, 1939

Siphotextularia catenata (Cushman, 1911)

(Pl. 12, fig. 7)

1911 Textularia catenata Cushman: 23, figs 39-40.

Occurrence. Late Miocene-Holocene; ODP holes 994C, 997A.

Genus Sphaeroidina d'Orbigny, 1826

Sphaeroidina bulloides (d'Orbigny, 1826)

(P1. 12, figs 8-9)

1826 Sphaeroidina bulloides d'Orbigny: 267, no.1, mod no. 65.

Occurrence. Late Miocene-Holocene; ODP holes 991A, 994C, 995A, 995B, 997A.

Genus Spirillina Ehrenberg, 1843

Spirillina decorata (Brady, 1884)

(Pl. 12, figs 10-11)

1884 Spirillina decorata Brady: 633, pl. 85, figs 22-25.
Occurrence. Late Miocene-Late Pleistocene; ODP holes 994C, 997A.

Genus Spiroloculina d'Orbigny, 1826

Spiroloculina esnaensis (LeRoy, 1953)

(Pl. 12, fig. 12)

1953 Spiroloculina esnaensis LeRoy: 49, pl. 3, figs 15-16.

Occurrence. Early Pliocene-Middle Pliocene; ODP holes 991A, 994C, 997A.

Spiroloculina tenuis (Czjzek, 1884)

(Pl. 12, figs 13-15)

1984 Sigmoilina tenuis (Czjzek); Brady: 21, pl. 10, figs 7-8, 11.

Occurrence. Early Pliocene-Early Pleistocene; ODP holes 994C, 995A, 997A.

Genus Stilostomella Guppy, 1894

Stilostomella abyssorum (Brady, 1884)

(Pl. 12, fig. 16)

1884 Nodosaria abyssorum Brady: 504, pl. 63, figs 8-9.

Occurrence. Late Miocene-Early Pleistocene; ODP holes 991A, 994C, 995A, 995B.

Stilostomella consobrina (d'Orbigny, 1846)

(Pl. 12, fig. 17)

1846 Dentalina consobrina d'Orbigny: 46, pl. 2, figs 1-3.

Occurrence. Late Miocene-Early Pleistocene; ODP holes 991A, 994C, 997A.

Stilostomella fistuca (Schwager, 1866)

(P1. 12, fig. 18)

1866 Nodosaria fistuca Schwager: 216, pl. 5, figs 36-37.

Occurrence. Late Miocene-Early Pleistocene; ODP holes 991A, 994C, 995A, 997A.

Stilostomella lepidula (Schwager, 1866)

(P1. 12, figs 19-20)

1866 Nodosaria lepidula Schwager: 210, pl. 5, fig. 27-28.

Occurrence. Late Miocene-Early Pleistocene; ODP holes 991A, 994C, 995A, 995B, 997A.

Genus Textularia Defrance, 1824

Textularia agglutinans (d'Orbigny, 1839)

(P1. 13, fig. 1) 


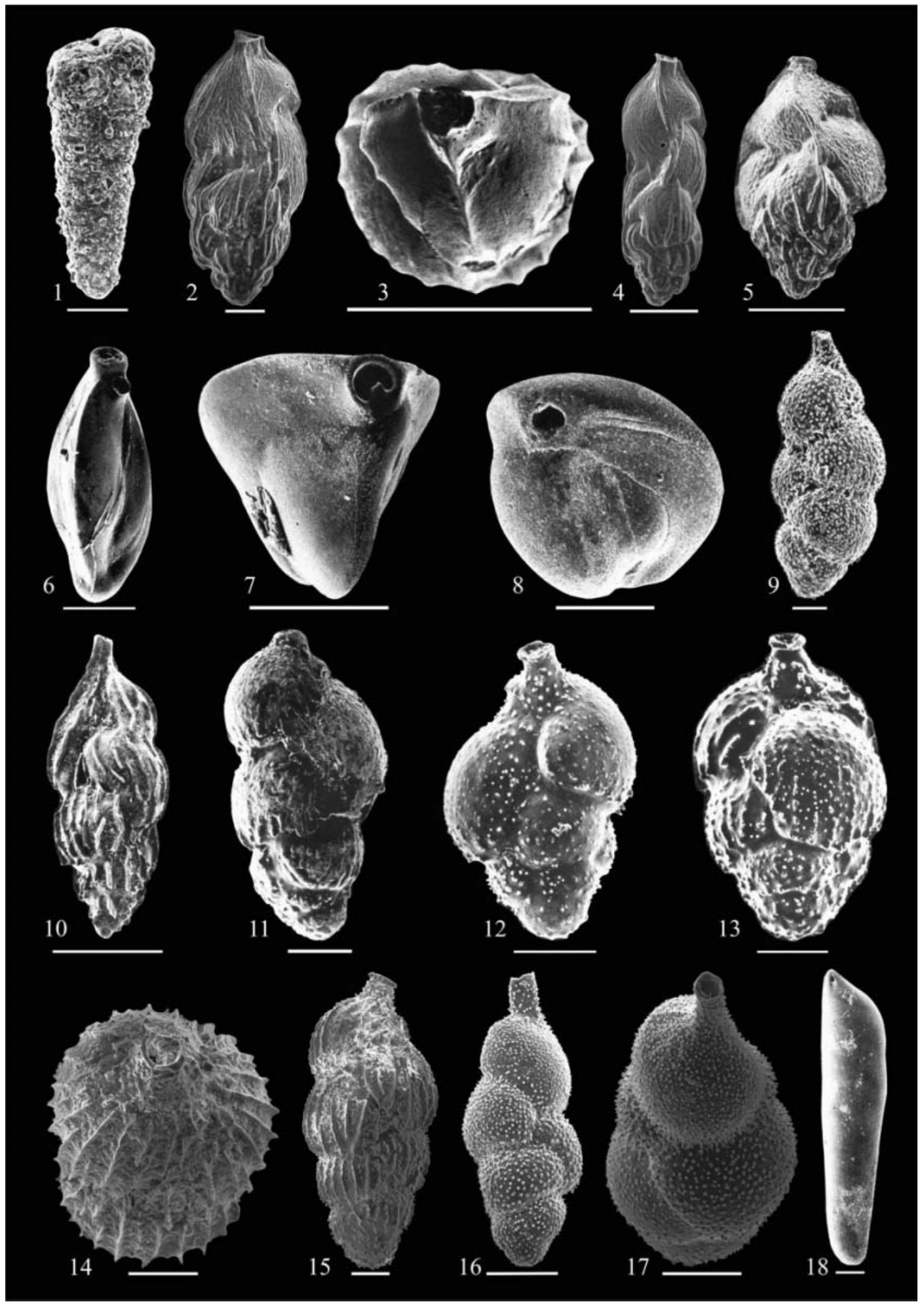

Explanation of Plate 13.

fig. 1. Textularia agglutinans, 164-994C, 34X-1, 4-6, side view, figs 2-4. Trifarina angulosa: 2, 4, 164-995A, 5H-6, 148-150, side view; 3, 164-994C, 42X-4, 51-53; apertural view. fig. 5. Trifarina reussi, 164-994C, 15H-2, 52-54, side view. figs 6, 7. Triloculina tricarinata, 164-994C, 25X-1, 75-77: 6, side view; 7, apertural view. fig. 8. Triloculina trigonula, 164-994C, 17H-2, 75-77, side view. fig. 9. Uvigerina auberiana, 164-994C, 35X-2, 52-54, side view. fig. 10. Uvigerina buzasi, 164-994C, 35X-CC, 0-2, side view. fig. 11. Uvigerina canariensis, 164-994C, 22X-4, 70-72, side view. fig. 12. Uvigerina hispida, 164-994C, 35X-2, 52-54, side view. fig. 13. Uvigerina hispido-costata, 164-994C, 40X-1, 54-56, side view. figs 14, 15. Uvigerina peregrina, 164-995A: 14, 6H-2, 75-77, apertural view; 15, 3H-2, 148-150, side view. figs 16, 17. Uvigerina proboscidea, 164-995A, 5H-6, 148-150: 16, side view; 17, apertural view. fig. 18. Vaginulina subelegans, 164-994C, 53P-1, 0-2, side view. Scale bar $50 \mu \mathrm{m}$ for fig. 2; $100 \mu \mathrm{m}$ for the rest. 
Deep-sea benthic foraminifera in the Neogene, NW Atlantic Ocean

1839 Textularia agglutinans d'Orbigny: 144, pl. 1, figs 17-18.

Occurrence. Late Miocene-Middle Pleistocene; ODP holes 994C, 995A, 997A.

Genus Trifarina Cushman, 1923

Trifarina angulosa (Williamson, 1858)

(P1. 13, figs 2-4)

1858 Uvigerina angulosa Williamson: 67, pl. 5, fig. 140.

Occurrence. Late Miocene-Holocene; ODP holes 991A, 994C, 995A, 995B, 997A.

\section{Trifarina reussi (Cushman, 1913)}

(P1. 13, fig. 5)

1913 Triplasia reussi Cushman: 63, pl. 3.

Occurrence. Early Pliocene-Holocene; ODP holes 991A, 994C, 995A, 995B.

Genus Triloculina d'Orbigny, 1826

Triloculina tricarinata (d'Orbigny, 1826)

(Pl. 13, figs 6-7)

1826 Triloculina tricarinata d'Orbigny: 299, mod no. 94.

Occurrence. Late Miocene-Holocene, ODP holes 991A, 994C, 995A, 995B, 997A.

Triloculina trigonula (Lamarck, 1804) (P1. 13, fig. 8)

1804 Miliolites trigonula Lamarck: 351, pl. 17, fig. 4.

Occurrence. Middle Pliocene; ODP holes 994C, 995A.

Genus Uvigerina d'Orbigny, 1826

Uvigerina auberiana (d'Orbigny, 1839)

(P1. 13, fig. 9)

1839 Uvigerina auberiana d'Orbigny: 106, pl. 2, figs 23-24.

Occurrence. Early Pliocene-Middle Pliocene; ODP hole 994C.

Uvigerina buzasi (Joseph \& Gupta, 1999)

(P1. 13, fig. 10)

1998 Uvigerina buzasi Joseph \& Gupta: pl. 1, figs 1-4.

Occurrence. Late Miocene-Late Pleistocene; ODP hole 997A.

Uvigerina canariensis (d'Orbigny, 1839)

(P1. 13, fig. 11)
1839 Uvigerina canariensis d'Orbigny: 138, pl. 1, figs 25-27.

Occurrence. Late Miocene-Late Pleistocene; ODP holes 991A, 994C, 995A, 995B.

Uvigerina hispida (Schwager, 1866)

(Pl. 13, fig. 12)

1866 Euvigerina hispida Schwager: 249, pl. 7, fig. 95.

Occurrence. Late Miocene-Late Pleistocene; ODP holes 991A, 994C, 995A, 995B, 997A.

Uvigerina hispido-costata (Cushman \& Todd, 1945) (Pl. 13, fig. 13)

1945 Uvigerina hispido-costata Cushman \& Todd: 51, pl. 7, figs $27,31$.

Occurrence. Late Miocene-Late Pleistocene; ODP holes 991A, 994C, 995A, 995B, 997A.

Uvigerina peregrina (Cushman, 1923)

(P1. 13, figs 14-15)

1923 Uvigerina peregrina Cushman: 166, pl. 42, figs 7-10.

Occurrence. Late Miocene-Holocene; ODP holes 991A, 994C, 995A, 995B, 997A.

Uvigerina proboscidea (Schwager, 1866)

(P1. 13, figs 16-17)

1866 Euvigerina proboscidea Schwager: 250, pl. 7, fig. 96.

Occurrence. Late Miocene-Holocene; ODP holes 991A, 994C, 995A, 995B, 997A.

Genus Vaginulina Silvestri, 1904

Vaginulina subelegans (Parr, 1950)

(Pl. 13, fig. 18)

1950 Vaginulina subelegans Parr: B.A.N.Z. Antartic Research Expedition, 1929-1931, Ser. B, 5(6), 325.

Occurrence. Late Miocene-Late Pleistocene; ODP holes 991A, 994C, 995A, 997A.

Manuscript received 19 April 2010

Manuscript accepted 27 January 2011

Scientific editing by Michal Kucera

\section{REFERENCES}

Akimoto, K., Hattori, M., Uematsu, K. \& Kato, C. 2001. The deepest living foraminifera, Challenger Deep, Mariana Trench. Marine Micropaleontology, 42: 95-97.

Almogi-Labin, A., Schmiedl, G., Hemleben, C., Siman-tov, R., Segl, M. $\&$ Meischner, D. 2000. The influence of the NE winter monsoon 
on productivity changes in the Gulf of Aden, NW Arabian Sea, during the last $530 \mathrm{ka}$ as recorded by foraminifera. Marine Micropaleontology, 40: 295-319.

Altenbach, A.V. \& Sarnthein, M. 1989. Productivity record in benthic foraminifera. In: Berger, W.H., Smetacek, V.S. \& Wefer, G. (Eds), Productivity of the oceans: present and past. Springer, New York, 255-269.

Arnold, A.J. 1983. Foraminiferal thanatocoenoses on the continental slope off Georgia and South Carolina. Journal of Foraminiferal Research, 13: 79-90.

Bailey, L.W. 1861. Notes on new species of microscopical organisms, chiefly from the Para River, South America. Boston Journal of Natural History, 7: 329-351.

Balsam, W.L. 1983. Carbonate dissolution on the Muir Seamount (Western North Atlantic): Interglacial/Glacial changes. Journal of Sedimentary Petrology, 53: 719-731.

Balsam, W.L. \& Damuth, J.E. 2000. Future investigations of shipboard vs. shore-based spectral data: Implication for interpreting Leg 164, sediment composition. Proceedings of the Ocean Drilling Program, Scientific Results, 164: 313-324.

Barker, R.W. 1960. Taxonomic notes on the species figured by H.B. Brady in his "Report on the foraminifera dredged by H.M.S. Challenger during the years 1873-1876". Society of Economic Paleontologists and Mineralogists, Special Publication, 9: 238pp.

Batsch, A.I.G.C. 1791. Sechs Kupfertafeln mit Conchylien des Seesandes, gezeichnet und gestochen von A.J.G.K. Batsch, Jena, pp. 3, 5.

Berggren, W.A., Hilgen, F.J., Langereis, C.G. et al. 1995a. Late Neogene chronology: New perspectives in high resolution stratigraphy. Geological Society of American Bulletin, 107: 1272-1287.

Berggren, W.A., Kent, D.V., Swisher, C.C. \& Aubry, M.P. 1995b. A revised Cenozoic geochronology and chronostratigraphy. Society of Economic Paleontologists and Mineralogists, Special Publication, 54: 129-212.

Bermúdez, P.J. 1937. Estudio micropaleontológico de dos formaciones eocénicas de las cercanıas de La. Habana, Cuba. Mem. Soc. Cubana Hist. Nat., 11, p. 238.

Bermùdez, P.J. 1949. Tertiary smaller foraminifera of the Dominican Republic Tertiary smaller foraminifera of the Dominican Republic. Cushman Laboratory for Foraminiferal Research, Special Publication, 25: 252.

Bernhard, J.M., Buck, K.R. \& Barry, J.P. 2001. Monterey Bay cold-seep biota: Assemblages, abundances, and ultrastructure of living foraminifera. Deep-Sea Research I, 48: 2233-2249.

Bhaumik, A.K. \& Gupta, A.K. 2007. Evidence of methane release from Blake Ridge ODP Hole 997A during the Plio-Pleistocene: Benthic foraminifer fauna and total organic carbon. Current Science, 92: 192-199.

Boersman, A. 1990. Late Oligocene to late Pliocene benthic foraminifers from depth traverses in the central Indian Ocean. In: Duncan, R.A., Backman, J. et al. (Eds), Proceedings of Ocean Drilling Program, Scientific Results, 115: 315-380.

Boetius, A., Ravenschlag, A., Schubert, C.J. et al. 2000. A marine microbial consortium apparently mediating anaerobic oxidation of methane. Nature, 407: 623-626.

Boltovskoy, E. 1978. Late Cenozoic deep-sea benthic foraminifera of the Ninetyeast Ridge (Indian Ocean). Marine Geology, 26: 139-175.

Bower, A.S. \& Hunt, H.D. 2000. Lagrangian Observation of the Deep Western Boundary Current in the North Atlantic Ocean, Part I: Large-Scale Pathways and Spreading Rates. Journal of Oceanography, 30: 764-783.

Brady, H.B. 1881. Notes on some of the Reticularian Rhizopoda of the "Challenger" Expedition. Part III. Quarterly Journal of Microscopical Science, 2: 31-71.

Brady, H.B. 1884. Report on the foraminifera dredged by H.M.S. Challenger, during the years 1873-1876. Reports of the scientific results of the voyage of H.M.S challenger, Zoology, 9: 1-814.

Burke, S.C., Berger, W.H., Coulbourn, W.T. \& Vincent, E. 1993 Benthic foraminifera in Box Core ERDC 112, Ontong Java Plateau. Journal of Foraminiferal Research, 23: 19-39.

Caralp, M.H. 1984. Quaternary calcareous benthic foraminifers, Leg 80. Initial Reports of the Deep Sea Drilling Project, 80: 725-755.
Caralp, M.H. 1988. Late Glacial to Recent Deep-Sea Benthic Foraminifera from the Northeastern Atlantic (Cadiz Gulf) and Western Mediterranean (Alboran Sea): Paleooceanographic Results. Marine Micropaleontology, 13: 265-289.

Caralp, M.H. 1989a. Size and morphology of the benthic foraminifer Melonis barleeanum: relationships with marine organic matter. Journal of Foraminiferal Research, 19: 235-245.

Caralp, M.H. 1989b. Abundance of Bulimina exilis and Melonis barleeanum: Relationship to the Quality of Marine Organic Matter. Geo-Marine Letters, 9: 37-43.

Chapman, F. 1916. Report of the Foraminifera and Ostracoda out of marine muds from soundings in the Ross Sea. In: Benson, W.N., Chapman, F., Cohen, F., Cotton, L.A., Hedley, C., Jensen, H.I., Mawson, D., Skeats, E.W., Thomson, J.A., Walkom, A.B. \& Woolnough, W.G., British Antarctic Expedition 1907-9 under the command of Sir E. H. Shackleton, C.V.O., Reports on the scientific investigations, Geology, Vol. 2, Contributions to the Palaeontology and Petrology of South Victoria Land: 53-80.

Chapman, F. 1941. Report on the foraminiferal sounding and dredgings of the F.I.S. "Endeavour" along the continental shelf of the south east coast of Australia. Transactions of the Royal Society of South Australia, 65: 145-211.

Chapman, F. \& Parr, W.J. 1937. Foraminifera Scientific Reports of the Australasian Antarctic Expedition, pp. 1-190.

Corliss, B.H. 1979. Recent deep-sea benthonic foraminiferal distributions in the southeast Indian Ocean: Inferred bottom water routes and ecological implications. Marine Geology, 31: 115-138.

Corliss, B.H. 1983. Distribution of Holocene deep-sea benthonic foraminifera in the southwest Indian Ocean. Deep-Sea Research A, 30: 95-117.

Corliss, B.H. 1985. Microhabitats of benthic foraminifera within deepsea sediments. Nature, 314: 435-438.

Corliss, B.H. \& Chen, C. 1988. Morphotype patterns of Norwegian Sea deep-sea benthic foraminifera and ecological implications. Geology, 16: 716-719.

Corliss, B.H. \& Emerson, S. 1990. Distribution of rose Bengal stained deep-sea benthic foraminifera from the Nova Scotia continental margin and Gulf of Maine. Deep Sea Research, 37: 381-400.

Costa, O.G. 1856. Paleontologia del regno di Napoli, parte II. Atti della Accademia Pontaniana, Napoli, 7: 113-378.

Coull, B.C., Ellison, R.L., Fleeger, J.W. et al. 1977. Quantitative estimates of the meiofauna from the deep sea off North Carolina, USA. Marine Biology, 39: 233-240.

Cushman, J.A. 1911. A monograph of the foraminifera of the North Pacific Ocean, part 2, Textulariidae. United States National Museum Bulletin, 72: 1-108.

Cushman, J.A. 1913. Textulariidae and other arenaceous Foraminifera from the Philippine Island and contiguous waters. United States National Museum Bulletin, 44: 633-638.

Cushman, J.A. 1915. A monograph of the foraminifers of the North Pacific Ocean: Part 5-Rotaliidae. United States National Museum Bulletin, 71: 1-81.

Cushman, J.A. 1922. Foraminifera of the Atlantic Ocean, Part 3. Textulariidae. United States National Museum Bulletin, 104: 1-143.

Cushman, J.A. 1923. The foraminifera of the Atlantic Ocean. United States National Museum Bulletin, 104: 1-228.

Cushman, J.A. 1927. Recent Foraminifera from off the west coast of America. Bulletin of the Scripps Institution of Oceanography, Technical Series 1: 119-188.

Cushman, J.A. 1929. The Foraminifera of the Atlantic Ocean. Part 6. Miliolidae, Ophthalmididae and Fischerinidae. United States National Museum Bulletin, 104: 1-129.

Cushman, J.A. 1933. Some new Recent foraminifera from the tropical Pacific. Contribution Cushman Laboratory Foraminifer Research, 9: 77-95.

Cushman, J.A. \& Hughes, D.D. 1925. Some later Tertiary Cassidulinas of California. Contributions Cushman Laboratory for Foraminiferal Research, 1: 11-17.

Cushman, J.A. \& Ozawa, Y. 1930. A Monograph of the Foraminiferal Family Polymorphinidae Recent and Fossil. United States National Museum Bulletin, 77: 1-195. 
Cushman, J.A. \& Parker, F.L. 1936. Notes on some Cretaceous species of Buliminella and Neobulimina. Contribution Cushman Laboratory Foraminifer Research, 12: 510.

Cushman, J.A. \& Parker, F.L. 1947. Bulimina and related Foraminiferal genera. United States Geological Survey Prof. Paper, 210-D: 55-176.

Cushman, J.A. \& Ponton, G.M. 1932. The foraminifera of the Upper, Middle and part of the Lower Miocene of Florida. Bulletin of Florida State Geological Survey, 9: 7-147.

Cushman, J.A. \& Todd, R. 1945. Miocene foraminifera from Buff Bay, Jamaica: Cushman Laboratory for Foraminiferal Research, Special Publication, 15: 1-73.

Cushman, J.A. \& Stainforth, R.M. 1945. The foraminifera of the Cipero Marl Formation of Trinidad, British West Indies: Cushman Laboratory for Foraminiferal Research, Special Publication, 14: 3-75.

De, S. \& Gupta, A.K. 2010. Deep-sea faunal provinces and their inferred environments in the Indian Ocean based on distribution of Recent benthic foraminifera. Palaeogeography, Palaeoclimatology, Palaeoecology, DOI: 10.1016/j.palaeo.2010.03.012.

De Rijk, S., Jorissen, F.J., Rohling, E.J. \& Troelstra, S.R. 2000. Organic flux control on bathymetric zonation of Mediterranean benthic foraminifera. Marine Micropaleontology, 40: 151-166.

De Stigter, H.C., Jorrisen, F.J. \& Van der Zwaan, G.J. 1998. Bathymetric distribution and microhabitat partitioning of live (rose bengal stained) benthic foraminifera along a shelf to deep sea transect in the southern Adriatic Sea. Journal of Foraminiferal Research, 28: 40-65.

Dervieux, E. 1899. Foraminiferi terziarii del piemonte e specialmente sul gen Polymorphina d'Orbigny. Bollettino della Societe Geologica Italiana, 18: 76-78.

d'Orbigny, A.D. 1826. Tableau méthodique de la classe des céphalopodes. Annales des Sciences Naturelles, Paris, série 1: 245-314.

d'Orbigny, A.D. 1839. Foraminifères de l'île de Cuba. In: de la Sagra, R. (Ed.), Histoire physique, politique et naturelle de l'île de Cuba. Arthus Bertrand, Paris, pp. 1-224.

d'Orbigny, A.D. 1846. Foraminifères fossiles du Bassin Tertiaire de Vienne (Austriche), Gide et Comp. Paris, pp. 1-312.

Earland, A. 1934. Foraminifera Part III. The Falklands sector of the Antarctic (excluding South Georgia). Discovery Reports, 10: 1-208.

Egger, J.G. 1893. Foraminiferen aus den Meeresgrundproben, gelothet von 1874 bis 1876 von S.M. Sch. Gazelle: Abhandlungen der Bayerischen Akademie der Wissenschaften, München, Math.-Phys. Cl., 18: 575-600.

Evans, H.K. \& Hall, I.R. 2008. Deepwater circulation on Blake Outer Ridge (western North Atlantic) during the Holocene, Younger Dryas, and Last Glacial Maximum. Geochemistry Geophysics Geosystems, 9, Q03023, 19, DOI: 10.1029/2007GC00 1771.

Fariduddin, M. \& Loubere, P. 1997. The surface ocean productivity response of deep water benthic foraminifera in the Atlantic Ocean. Marine Micropaleontology, 32: 289-310.

Feyling-Hanssen, R.W. 1954. Late Pleistocene foraminifera from the Oslofjord area, Southeast Norway: Norsk Geologisk Tidsskrift, 33: 109-152.

Fichtel, L. von. \& Moll, J.P.C.von. 1798. Testacea microscopica, aliaque minuta ex generibus Argonauta et Nautilus, ad naturam picta et descripta: Camesina, Vienna.

Fontanier, C., Jorissen, F.J., Licari, L., Alexandre, A., Anschutz, P. \& Carbonel, P. 2002. Live benthic foraminiferal faunas from the Bay of Biscay: faunal density, composition, and microhabitats. Deep-Sea Research I, 49: 751-785.

Fontanier, C., Jorissen, F.J., Chaillou, G., David, C., Anschutz, P. \& Lafon, V. 2003. Seasonal and interannual variability of benthic foraminiferal faunas at $550 \mathrm{~m}$ depth in the Bay of Biscay. Deep-Sea Research I, 50: 457-494.

Fontanier, C., Jorissen, F.J., Chaillou, G., Anschutz, P., Grémare, A. \& Griveaud, C. 2005. Live foraminiferal faunas from a $2800 \mathrm{~m}$ deep lower canyon station from the Bay of Biscay: Faunal response to focusing of refractory organic matter. Deep-Sea Research I, 52: $1189-1227$.

Frank, M., Whiteley, N., Kasten, S., Hein, R. \& O’Nions, K. 2002. North Atlantic Deep Water export to the Southern Ocean over the past 14 Myr: Evidence from $\mathrm{Nd}$ and $\mathrm{Pb}$ isotopes in ferromanganese crusts. Paleoceanography, 17, DOI: 10.1029/2000 PA000606.
Franz, S.O. \& Tiedemann, R. 2002. Depositional changes along the Blake-Bahama Outer Ridge deep water transect during marine isotope stages 8 to 10 - links to the Deep Western Boundary Current. Marine Geology, 189: 107-122.

Friedrich, O. \& Hemleben, C. 2007. Early Maastrichtian benthic foraminiferal assemblages from the western North Atlantic (Blake Nose) and their relation to paleoenvironmental changes, Marine Micropaleontology, 62: 31-44.

Geslin, E., Heinz, P., Jorissen, F. \& Hemleben, Ch. 2004. Migratory response of deep-sea benthic foraminifera to variable oxygen conditions: laboratory investigations. Marine Micropaleontology, 53: 227-243.

Gibson, G.W. 1967. Foraminifera and stratigraphy of the Tongaporutuan Stage in the Taranaki coastal and six other sections. Part-I: Systematics and distribution: Transactions of the Royal Society of New Zealand, Geology, 5: 1-70.

Goës, A. 1894. A synopsis of the Arctic and Scandinavian recent marine Foraminifera hitherto discovered. Kongl. Svenska VetenskapsAkademiens Handlingar, 25: 1-127.

Gooday, A.J. 1988. A response by benthic foraminifera to the deposition of phytodetritus in the deep sea. Nature, 332, 70-73.

Gooday, A.J. 1993. Deep-sea benthic foraminiferal species, which exploit phytodetritus: characteristic features and controls on distribution. Marine Micropaleontology, 22: 187-205.

Gooday, A.J. 1994. The biology of deep-sea foraminifers: A review of some advances and their applications in Paleoceanography. Palaios, 9: 14-31.

Gooday, A.J. 2003. Benthic Foraminifera (Protista) as Tools in DeepWater Paleoceanography: Environmental influences on Faunal Characteristics. Advances in Marine Biology, 46: 1-90.

Guppy, R.J.L. 1894. On the microzoa of the Tertiary and other rocks of Trinidad and the West Indies. Journal of the Trinidad Field Naturalists' Club, 1: 277-290.

Gupta, A.K. 1993. Biostratigraphic vs. paleoceanographic importance of Stilostomella lepidula (Schwager) in the Indian Ocean. Micropaleontology, 39(1): 47-52.

Gupta, A.K. 1994. Taxonomy and bathymetric distribution of Holocene deep-sea benthic foraminifera in the Indian Ocean and the Red Sea. Micropaleontology, 40: 351-367.

Gupta, A.K. 1997. Paleoceanographic and paleoclimatic history of the Somali Basin during the Pliocene-Pleistocene: Multivariate analyses of benthic foraminifera from DSDP Site 241 (leg 25). Journal of Foraminiferal Research, 27: 196-208.

Gupta, A.K. \& Satapathy, S.K. 2000. Latest Miocene-Pleistocene abyssal benthic foraminifera from west-central Indian Ocean DSDP Site 236: Paleoceanographic and paleoclimatic inferences. Journal of Paleontological Society of India, 45: 33-48.

Gupta, A.K. \& Srinivasan, M.S. 1992. Uvigerina proboscidea abundance and paleoceanography of the northern Indian Ocean DSDP Site 214 during the Late Neogene. Marine Micropaleontology, 19: 355-367.

Gupta, A.K. \& Srinivasan, M.S. 1996. Multivariate analyses of Pliocene-Pleistocene benthic foraminifera from DSDP Site 214, eastern Indian Ocean: paleoceanic and paleoclimatic implications. In: Pandey, J., Azmi, R.J., Bhandari, A. \& Dave, A. (Eds), Contributions to XV Indian Colloquium on Micropaleontology and Stratigraphy. Dehra Dun, 339-355.

Gupta, A.K. \& Thomas, E. 1999. Latest Miocene-Pleistocene productivity and deep-sea ventilation in the northwestern Indian Ocean (Deep Sea Drilling Project Site 219). Paleoceanography, 14: 62-73.

Gupta, A.K. \& Thomas, E. 2003. Initiation of Northern Hemisphere glaciation and strengthening of the northeast Indian monsoon: Ocean Drilling Program Site 758, eastern equatorial Indian Ocean. Geology, 31: 47-50.

Hantken, M.von. 1875. Die fauna der Clavulina Szaboi-Schichten. Thiel I, Foraminiferen: Kaiserlich Ungarische Anstalt, Mitteilungen, Jahrbuch, 4: 1-93.

Haug, G.H. \& Tiedemann, R. 1998. Effect of the formation of the Isthmus of Panama on Atlantic Ocean thermohaline circulation. Nature, 393: 673-676.

Hayward, B.W. 2001. Global deep-sea extinctions during the Pleistocene ice ages. Geology, 29: 599-602. 
Hayward, B.W. 2002. Late Pliocene to middle Pleistocene extinctions of deep-sea benthic foraminifera ("Stilostomella extinction") in the southwest Pacific. Journal of Foraminiferal Research, 32: 274-307.

Hayward, B.W., Sabaa, A.T., Thomas, E. et al. 2010. Cenozoic record of elongate, cylindrical, deep-sea benthic foraminifera in the Indian Ocean (ODP Sites 722, 738, 744, 758, and 763). Journal of Foraminiferal Research, 40: 113-133.

Hermelin, J.O.R. \& Shimmield, G.B. 1990. The importance of the oxygen minimum zone and sediment geochemistry in the distribution of recent benthic foraminifera in the northwest Indian Ocean. Marine Geology, 91: 1-29.

Heron-Allen, E. \& Earland, A. 1922. Protozoa, Part 2: Foraminifera of the British Antarctic ('Terra Nova') Expedition, 1910. Natural History Report, Zoology 6, 137.

Hessland, I.R. 1943. Marine Schalenablagerungen Nord-Bohuslans. Bulletin of the Geological Institution of the University of Upsala, 31 $1-348$.

Höglund, H. 1947. Foraminiferain the Gullmar Fjord and the Skagerak. Zoologiske Bidrag från Uppsala, 26: 273.

Jannink, N.T., Zachariasse, W.J. \& Van der Zwaan, G.J. 1998. Living (rose Bengal stained) benthic foraminifera from the Pakistan continental margin (Northern Arabian Sea). Deep-Sea Research I, 45: 1483-1513.

Jones, R.W. 1994. The Challenger Foraminifera. Oxford University Press, USA, 149pp.

Jorissen, F.J., Barmawidjaja, D.M., Puskaric, S. \& Van der Zwaan, G.J. 1992. Vertical distribution of benthic foraminifera in the Northern Adriatic Sea: the relation with high organic flux. Marine Micropaleontology, 19: 131-146.

Jorissen, F.J., De Stigter, H.C. \& Widmark, J.G.V. 1995. A conceptual model explaining benthic foraminiferal microhabitats. Marine Micropaleontology, 26: 3-15.

Joseph, S. \& Gupta, A.K. 1999. New species of benthic Foraminifera from the Neogene sequence of ODP sites 757B and 758A, northeastern Indian Ocean. Micropaleontology, 45: 221-224.

Karrer, F.W. 1878. Die Foraminiferen der Tertiaren Thone von Luzon In: von Drasche, R., Fragmente zu einer Geologie der Insel Luzon (Philippinen), pp. 75-99.

Kawagata, S., Hayward, B.W., Grenfell, H.R. \& Sabba, A.T. 2005. Mid-Pleistocene extinction of deep-sea foraminifera in the North Atlantic Gateway (ODP Sites 980 and 982). Palaeogeography Palaeoclimatology Palaeoecology, 221: 267-291.

Kawagata, S., Hayward, B.W. \& Gupta, A.K. 2006. Benthic foraminiferal extinctions linked to late Pliocene-Pleistocene deep-sea circulation changes in the northern Indian Ocean. Marine Micropaleontology, 58: $219-242$.

Kim, S.J. \& Crowley, T.J. 2000. Increased Pliocene North Atlantic Deep Water: Cause or Consequence of Pliocene Warming? Paleoceanography, 15: 451-455.

Kurbjeweit, F., Schmiedl, G., Schiebel, R., Hemleben, Ch., Pfannkuche, O., Wallmann, K. \& Schaefer, P. 2000. Distribution, biomass and diversity of benthic foraminifera in relation to sediment geochemistry in the Arabian Sea. Deep-Sea Research II, 47: 2913-2955.

Laine, E.P., Gardner, W.D., Richardson, M.J. \& Kominz, M.A. 1994 Abyssal currents and advection of resuspended sediment along the northeastern Bermuda Rise. Marine Geology, 119: 159-171.

Lamarck, J.B. 1804. Suite des mémoires sur les fossiles des environs de Annales Muséum National Histoire Naturelle, 5: 237-245.

Ledbetter, M.T. \& Balsam, W.L. 1985. Paleoceanography of the deep Western Boundary Undercurrent on the North American continental margin for the last 25,000 years. Geology, 13: 181-184.

LeRoy, L.W. 1944. Miocene Foraminifera from Sumatra and Java, Netherlands East Indies: Ibid., 39: 1-113.

LeRoy, L.W. 1953. Biostratigraphy of the Ain Maqfi Section, Egypt Geological Society of America, Memoir, 54: 1-73.

Linnæus, C. 1758. Systema naturæ per regna tria naturæ, secundum classes, ordines, genera, species, cum characteribus, differentiis, synonymis, locis. Tomus I. Editio decima, reformata, Holmiæ (Salvius): $1-824$.
Lobegeier, M.K. \& Sen. Gupta, B.K. 2008. Foraminifera of hydrocarbon seeps, Gulf of Mexico. Journal of Foraminiferal Research, 38: 93-116.

Loeblich, A.R. \& Tappan, H. 1953. Studies of Arctic Foraminifera. Smithsonian Miscellaneous Collection, 121.

Loeblich, A.R. \& Tappan, H. 1964. Treatise on Invertibrate Paleontology. Part C, vols 1 \& 2. The Geological Society of America and The University of Kansas Press.

Loeblich, A.R. \& Tappan, H. 1988. Foraminiferal genera and their classification. Van Nostrand Reinhold Company, New York, vols 1 \& 2, 970pp.

Loubere, P. 1991. Deep-sea benthic foraminiferal assemblage response to a surface ocean productivity gradient: a test. Paleoceanography, $\mathbf{6}$ : 193-204.

Loubere, P. 1994. Quantitative estimation of surface ocean productivity and bottom water oxygen concentration using benthic foraminifera. Paleoceanography, 9: 723-737.

Loubere, P. 1998. The impact of seasonality on the benthos as reflected in the assemblages of deep-sea foraminifera. Deep-sea Research, I, 45: 409-432.

Loubere, P. \& Fariduddin, M. 1999. Quantitative estimates of global patterns of surface ocean biological productivity and its seasonal variation on time scales from centuries to millennia. Global Biogeochemical Cycles, 13: 115-133.

Lutze, G.F. \& Coulbourn, W.T. 1984. Recent benthic foraminifera from the continental margin of Northwest Africa: community structure and distribution. Marine Micropaleontology, 8: 361-401.

Maas, M. 2000. Verbreitung lebendgefärbter benthischer Foraminiferen in einer intensivierten Sauerstoffminimumzone, Indo-Pakistanischer Kontinentalrand, nördliches Arabisches Meer [Distribution of Rose Bengal stained benthic foraminifera within an intensified oxygen minimum zone, Indo-Pakistan Continental Margin, Northwest Arabian Sea]. Meyniana, 52: 101-128.

Macfadyen, W.A. 1932. Foraminifera from some Late Pliocene and glacial deposits of East Anglia. Geological Magazine, 69: 481-497.

Mackensen, A., Schmiedl, G., Harloff, J. \& Giese, M. 1995. Deep-sea foraminifera in the South Atlantic Ocean: Ecology and assemblage generation. Micropaleontology, 41: 342-358.

Markl, R.G., Bryan, G.M. \& Ewing, J.I. 1970. Structure of the Blake-Bahama Outer Ridge. Journal of Geophysical Research, 75: 4539-4555.

Miller, K.G. \& Lohmann, G.P. 1982. Environmental distribution of recent benthic foraminifera on the northeast United States continental slope. Geological Society of America Bulletin, 93: 200-206.

Montagu, G. 1803. Testacea Britannica, or natural history of British shells, marine, land and fresh-water, including the most minute. J.S. Hollis, Romsey, England: 1-606.

Murgese, D.S. \& De. Deckker, P. 2005. The distribution of deep-sea benthic foraminifera in core tops from the eastern Indian Ocean. Marine Micropaleontology, 56: 25-49.

Nees, S. \& Struck, U. 1999. Benthic foraminiferal response to major paleoceanographic changes. In: Abrantes, F. \& Mix, A. (Eds), Reconstructing Ocean History: $A$ Window into the Future. Kluwer Academic Plenum Publishers, New York, 195-216.

Ohkushi, K., Thomas, E. \& Kawahata, H. 2000. Abyssal benthic foraminifera from the northwestern Pacific (Shatsky Rise) during the last 298 kyr. Marine Micropaleontology, 38: 119-147.

Okada, H. 2000. Neogene and Quaternary calcareous nannofossils from the Blake Ridge, Sites 994, 995, and 997. In: Paull, C.K., Mutsumoto, R., Wallace, P.J. \& Dillon, W.P. (Eds), Proceedings of the Ocean Drilling Program, Scientific Results, 164: 331-341.

Panieri, G. 2005. Benthic foraminifera associated with a hydrocarbon seep in the Rockall Trough (NE Atlantic). Geobios, 38: 247-255.

Panieri, G. \& Sen.Gupta, B.K. 2008. Benthic foraminifera of the Blake Ridge hydrate mound, western North Atlantic Ocean. Marine Micropaleontology, 66: 91-102.

Parker, F.L. 1958. Eastern Mediterranean Foraminifera. Reports of the Swedish Deep-sea Expedition (1947-1948), 8: 217-283.

Parker, W.K., Jones, T.R. \& Brady, H.B. 1871. On the nomenclature of the foraminifera. Pt. XIV. The species enumerated by d'Orbigny in the 
"Annales des Sciences Naturelles" vol. vii. 1826 (4). Annals and Magazine of Natural History, 48: 145-179, 236-266.

Parker, W.K. \& Jones, T.R. 1865. On some foraminifera from the North Atlantic and Arctic Oceans, including Davis Strait and Baffin's Bay. Royal Society of London, Philosophical Transactions, 155: 325-341.

Parr, W.J. 1950. Foraminifera Reports of the British Australian and New Zealand Antarctic Research Expedition (1929-1931), series B (Zoology and Botany) 5: 233-392.

Paull, C.K., Matsumoto, R., Wallace, P.J. \& Shipboard Scientific Party 1996. Sites 991-997. Proceedings of the Ocean Drilling Program, Initial Reports, 164: 65-334.

Peterson, L.C. 1984. Recent abyssal benthic foraminiferal biofacies of the east equatorial Indian Ocean. Marine Micropaleontology, 8: 479-519.

Phleger, F.B., Parker, F.L. \& Peirson, J. 1953. Sediments cores from the North Atlantic Ocean. Reports of the Swedish Deep-sea Expedition, 8: $1-12$.

Phleger, F.B. \& Parker, F.L. 1951. Ecology of foraminifera, Northwest Gulf of Mexico, Part II: foraminifera species. Geological Society of America, Memoir, 46: 1-64.

Qvale, G \& Van. Weering, T.C.F. 1985. Relationship of surface sediments and benthic foraminiferal distribution patterns in the Norwegian Channel (Northern North Sea). Marine Micropaleontology, 9: 469-488.

Rathburn, A.E. \& Corliss, B.H. 1994. The ecology of living (stained) benthic foraminifera from the Sulu Sea. Paleoceanography, 9: 87-150.

Rathburn, A.E., Levin, L.A., Held, Z. \& Lohmann, K.C. 2000. Benthic foraminifera associated with cold methane seeps on the northern California margin: Ecology and stable isotopic composition. Marine Micropaleontology, 38: 247-266.

Rau, W.W. 1948. Foraminifera from the Porter shale (Lincoln formation), Grays Harbor County, Washington: Journal of Paleontology, 22: $152-174$.

Raymo, M.E., Ruddiman, W.F., Shackleton, N.J. \& Oppo, D. 1990. Evolution of Atlantic-Pacific $\delta^{13} \mathrm{C}$ gradients over the last $2.5 \mathrm{~m} . \mathrm{y}$. Earth and Planetary Science Letters, 97: 353-368.

Raymo, M.E., Grant, B., Horowitz, M. \& Rau, G.H. 1996. MidPliocene warmth: stronger greenhouse and stronger conveyor. Marine Micropaleontology, 27: 313-326.

Renz, H.H. 1948. Stratigraphy and fauna of the Agua Salada Group, State of Falcón, Venezuela. Mem. Geological Society of America, 32: $1-219$.

Reuss, A.E. 1850. Neue foraminiferen aus den Schichten des österreichischen Tertiärbeckens. Denkschr. Akad. Wiss., Wien, math-naturwiss, 1: $365-390$.

Reuss, A.E. 1851. Über die fossilen Foraminiferen und Entomostracean der Septarienthone der umgegend von Berlin. Zeitschrift der Deutschen Geologischen Gesellschaft, 3: 49-92.

Reynolds, B.C., Frank, M. \& O’Nions, R.K. 1999. Nd- and Pb- isotope time series from Atlantic ferromanganese crusts: Implications for changes in provenance and paleocirculation over the last 8 Myr. Earth and Planetary Science Letters, 173: 381-396.

Robinson, C.A., Bernhard, J.M., Levin, L.A., Mendoza, G.F. \& Blanks, J.K. 2004. Surficial Hydrocarbon Seep Infauna from the Blake Ridge (Atlantic Ocean, 2150 m) and the Gulf of Mexico (690-2240 m). Marine Ecology, 25, 313-336.

Roemer 1838. Neues Jahr. Mineral. Geogr. Geol. And PeterefaktenKunde, p. 386.

Schlumberger, C. 1891. Revision des biloculines des grands fonds. Mem. Soc. Zool. France, 4: 542-579.

Schmiedl, G. \& Mackensen, A. 1997. Late Quaternary paleoproductivity and deep water circulation in the eastern South Atlantic Ocean: Evidence from benthic foraminifera. Palaeogeography, Palaeoclimatology, Palaeoecology, 130: 43-80.

Schmiedl, G., Mackensen, A. \& Müller, P.J. 1997. Recent benthic foraminifera from the eastern South Atlantic Ocean: dependence on food supply and water masses. Marine Micropaleontology, 32: 249-287.

Schnitker, D. 1980. Quaternary deep-sea benthic foraminifers and water masses. Annual Review of Earth and Planetary Sciences, 8: 343-370.
Schubert, R.J. 1911. Die fossilen foraminiferen des Bismarckarchipels und eineger angrenzender Inseln. Abhandlungen der geologischen Reischsanstalt, 20: 1-130.

Schumacher, S., Jorissen, F.J., Dissard, D., Larkin, K.E. \& Gooday, A.J. 2007. Live (Rose Bengal stained) and dead benthic foraminifera from the oxygen minimum zone of the Pakistan continental margin (Arabian Sea). Marine Micropaleontology, 62: 45-73.

Schwager, C. 1866. Fossile Foraminiferen von Kar Nikobar, Reise der Oesterreichischen Fregatte Novara um Erde in den Jahren 1857, 1858, 1859 unten den Befehlen des Commodore B. Von WuellerstorfUrbair. Geologischer Theil, Geologische Beobachtung no. 2, Palaeontologische Mittheilung, 2: 187-268.

Schwager, C. 1878. Nota su alcuni foraminiferi nuovi del tufo di stretto presso girenti. Bolletino R. Comitato Geologico d'Italia, 9: 511-529.

Seguenza, G. 1862. Dei terreni terziarii del distretto di Messina: Part II. Descrizione dei foraminiferi monotalamici delle marne Mioceniche del distretto di Messina. T. Capra, Messina, Italia.

Sen. Gupta, B.K. \& Aharon, P. 1994. Benthic foraminifera of bathyal hydrocarbon vents of the Gulf of Mexico: Initial report on communities and stable isotopes. Geo-Marine Letters, 14: 88-96.

Sen. Gupta, B.K. \& Machain-Castillo, L.M. 1993. Benthic foraminifera in oxygen-poor habitats. Marine Micropaleontology, 20: 183-201.

Shackleton, N.J., Imbrie, J. \& Hall, M.A. 1983. Oxygen and Carbon Isotope Record of East Pacific Core V19-30: Implications for the Formation of Deep Water in the Late Pleistocene North Atlantic. Earth and Planetary Science Letters, 65: 233-244.

Silvestri, O. 1872. Saggio di studi sulla fauna microscopia fossile appartenente al terreno subappenino italiano. Mem. I-monografia delle Nodosarie: Academia Gioenia Scienze Naturali Catania, Atti. 7: 108.

Silvestri, A. 1896. Foraminiferi pliocenici di Siena. Part-I: Memorie dell' Accademia Pontificia dei Nuovi Lincei, 12: 1-224.

Silvestri, A. 1900. Sur genere Ellipsoglandulina: Atti e Rendiconti R. Accademnia di Scienze. Lettere ed Arte degli Zelanti, Acireale, Cl. Sci. Mem. 10: 1-9.

Silvestri, A. 1903. Alcune osservazioni sui Protozoi fossili piemontesi. Atti della Reale Accademia delle Scienze di Torino, 38: 206-217.

Silvestri, A. 1904. Richerche strutturali su alcune forme dei Trubi dei Bonfornello (Palermo): Memorie dell'Accademia Pontificia dei Nuovi Lincei, 22: 235-276.

Singh, R.K. \& Gupta, A.K. 2004. Late Oligocene-Miocene paleoceanographic evolution of the southeastern Indian Ocean: Evidence from deep-sea benthic foraminifera (ODP Site 757). Marine Micropaleontology, 51: 153-170.

Singh, R.K. \& Gupta, A.K. 2005. Systematic decline in benthic foraminiferal species diversity linked to productivity increases over the last $26 \mathrm{Ma}$ in the Indian Ocean. Journal of Foraminiferal Research, 35: 219-227.

Srinivasan, M.S. \& Sharma, V. 1980. Schwagers Car Nicobar Foraminifera in the Reports of the 'Novara' Expedition - Revision. Today and Tomorrow' Printers and Publishers, New Delhi, pp. 1-83.

Stache 1864. Die foraminiferen der Tertiaren Mergel des Whain-garoaHafens (prov. Auckland). Novara Expedition, 2: 204.

Stahr, F.R. \& Sanford, T.B. 1999. Transport and bottom boundary layer observations of the North Atlantic Deep Western Boundary Current at the Blake Outer Ridge. Deep-Sea Research, II, 46: 205-243.

Stewart, R.E. \& Stewart, K.C. 1930. Post-Miocene Foraminifera from the Ventura quadrangle, Ventura County California. Journal of Paleontology, 4: 60-72.

Tappan, H. 1951. Northern Alaskan index foraminifera. Contributions from the Cushman Foundation for Foraminiferal Research, 2: 7.

Thalmann, H.E. 1937. Mitteilungen über Foraminiferen III: Eclogae Geologicae Helvetiae, 30: 337-356.

Thomas, E., Booth, L., Maslin, M. \& Shackleton, N.J. 1995. Northeastern Atlantic benthic foraminifera during the last 45,000 years: changes in productivity seen from the bottom up. Paleoceanography, 10: 545-562.

Trauth, F. 1918. Das Eozanvorkommen bei Radstadt im Pongou und seine Beziehungen zu den gleichalterigen Ablagerungen bei Kirchberg 
am Wechsel und Wimpassing am Leithagebirge. Kaiserliche Akademie der Wissenschaften, Mathematisch-Naturwissenschaftliche Classe, Denkschriften, Wien, 95: 171-278.

Tucholke, B.E. \& Mountain, G.S. 1979. Seismic stratigraphy, lithostratigraphy and paleosedimentation patterns in the North American Basin. In: Talwani, M., Hay, W. \& Ryan, W.B.F. (Eds), Deep Drilling Results in the Atlantic Ocean: Continental Margins and Paleoenvironment. American Geophysical Union Maurice Ewing Series, 3: $58-86$.

Uchio, T. 1952. Geology of Natural gas in the western Tart of Mobara-Machi, Chiba Prefectura (Japanese with English summary). Journal of the Japanese Association for Petroleum Technology, 17: 36.

Van der Zwaan, G.J., Jorissen, F.J. \& De Stigter, H.C. 1990. The depth dependency of planktonic/benthonic foraminiferal ratios: constraints and applications. Marine Geology, 95: 1-16.
Van der Zwaan, G.J., Duijnstee, I.A.P., den Dulk, M., Ernst, S.R., Jannink, N.T. \& Kowenhoven, T.J. 1999. Benthic foraminifers: proxies or problems? A review of paleoecological concepts. Earthscience Reviews, 46: 213-236.

Wefer, G., Heinze, P.M. \& Berger, W.H. 1994. Clues to ancient methane release. Nature, 369: 282.

Williamson, W.C. 1858. On the Recent Foraminifera of Great Britain. Ray Society, London.

Wood, W.T. \& Ruppel, C. 2000. Seismic and thermal investigations of the Blake Ridge gas hydrate area: A synthesis. In: Paull, C.K., Matsumoto, R. \& Dillon, W.P. (Eds), Proceedings of the Ocean Drilling Program, Scientific Results, 164: 253-264.

Zachos, J., Pagani, M., Sloan, L., Thomas, E. \& Billups, K. 2001. Trends, Rhythms, and Aberrations in Global Climate $65 \mathrm{Ma}$ to Present. Science, 292: 686-693. 